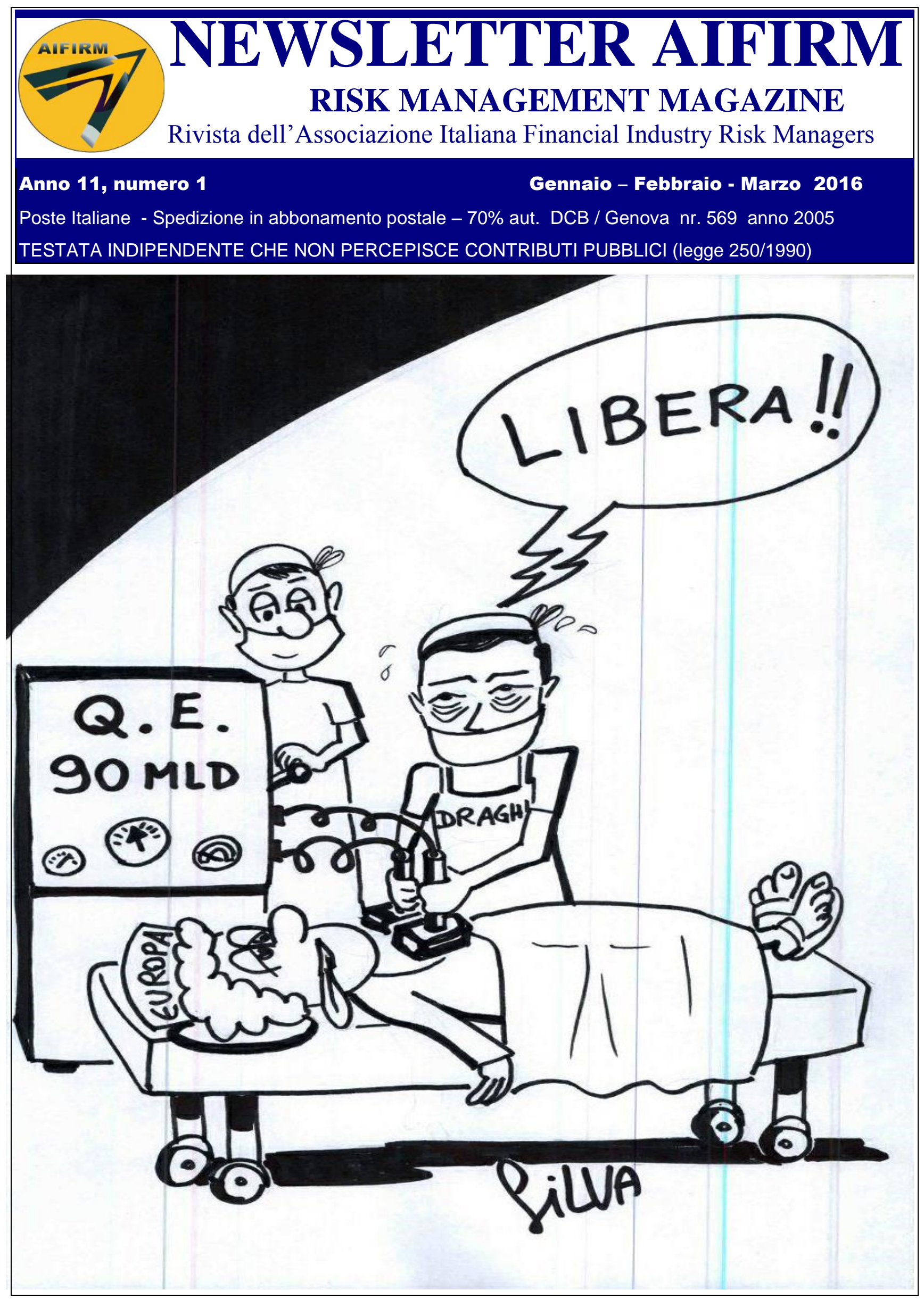

NEWSLETTER AIFIRM RISK MANAGEMENT MAGAZINE ANNO 11 ํ 1 - PAGINA - 1 - 


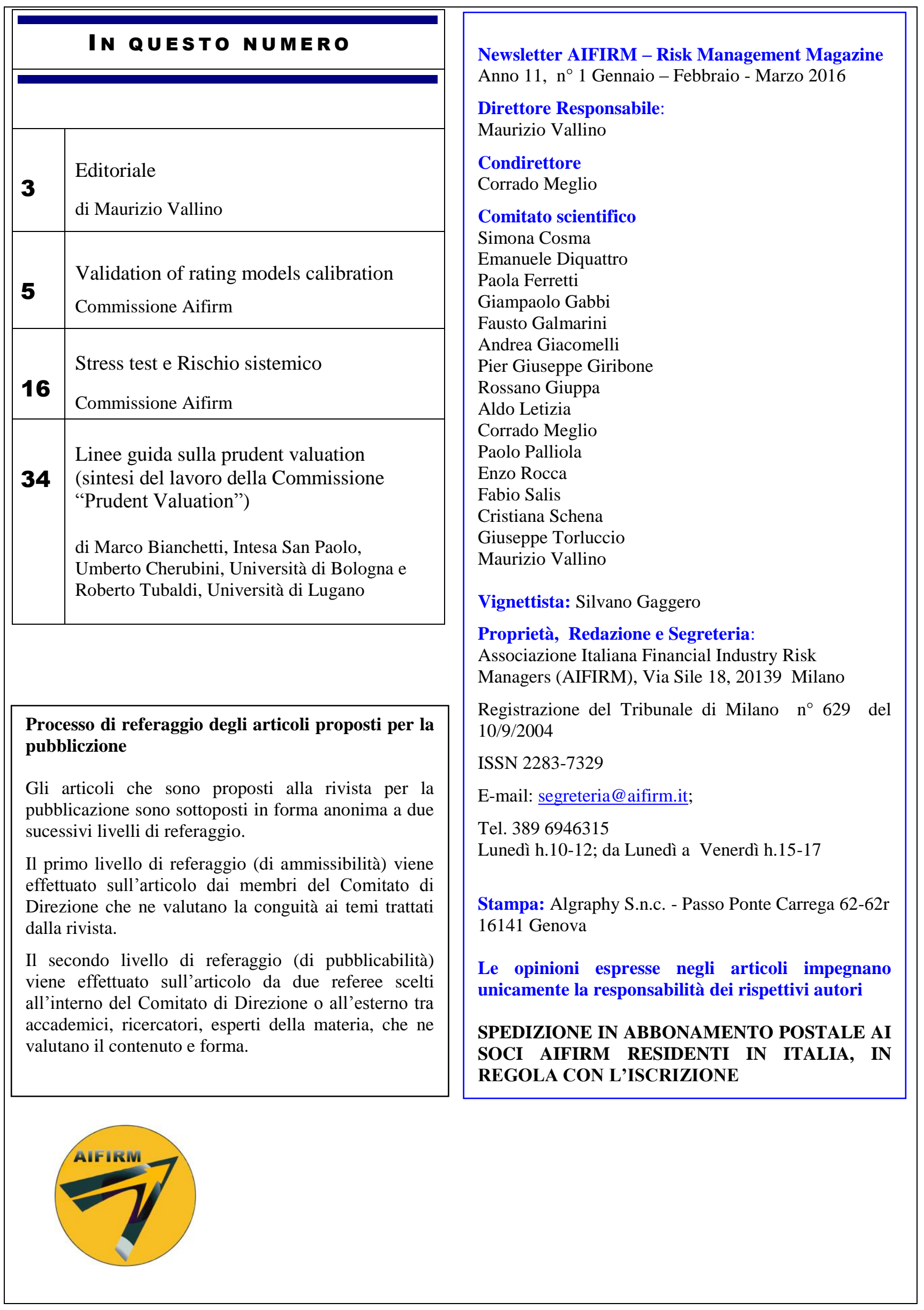




\section{EDITORIALE}

Cari lettori,

l'attuale numero della nostra rivista ha un significato particolare per AIFIRM in quanto corona il progetto dell' Associazione di essere non solo un luogo di scambio e confronto tra i soci ma anche, e soprattutto, uno strumento di ricerca nel campo del Risk Management.

Sono lieto di comunicare che sono giunti a conclusione i lavori di tre Commissioni Aifirm:

- Validation of rating models calibration

- Stress test e Rischio sistemico

- Prudent Valuation

che hanno prodotto altrettanti documenti che il Consiglio di Aifirm ha appena provveduto ad approvare, conferendo loro l'attribuzione Position Paper di AIFIRM.

Pubblichiamo in questo numero i primi due lavori e una sintesi (per ragioni di spazio) del terzo lavoro, i lavori integrali sono disponibili sul sito www.aifirm.it.

Nella seduta del 21 dicembre il Cosiglio dell'Associazione ha, inoltre, deliberato l'avvio di due ulteriori Commissioni:

- L'impatto dell'entrata in vigore degli standard IFRS9 (coordinatore accademico Andrea Resti);

- Il ruolo del RAF nella Governance delle banche (coordinatori accademici Marina Brogi e Antonio Ricciardi)

che prenderanno avvio, rispettivamente, nei mesi di aprile e di maggio.

Le nuove commissioni avranno una nuova struttura organizzativa che prevede, oltre ai coordinatori accademici e Aifirm, anche la presenza di una società di consulenza che avrà il ruolo di PMO e faciliterà la gestione dei tempi e la finalizzazione del lavoro.

Maurizio Vallino 


\section{Validation of rating models calibration}

\section{Position paper di AIFIRM ${ }^{1}$}

\section{Abstract}

A causa dell'attuale recessione, in fase di validazione dei sistemi di rating, molte Banche si trovano ad affrontare problemi di sottostima dei tassi di default osservati per alcuni segmenti di clientela, che si concretizzano in frequenti failures dei test di calibrazione "classici", quali i test binomiali.

Le ragioni che possono portare a tali risultati, anche per un sistema di rating performante, sono molteplici, ma le più critiche sono la filosofia del sistema di rating in validazione e la correlazione tra default.

Per una migliore comprensione delle filosofie sottostanti, ovvero dei sistemi di rating point in time (PIT) o through the cycle (TTC), è necessario distinguere due concetti di "ciclicità": uno si riferisce al processo di ordinamento delle controparti e quindi al processo di assegnazione dello score, in termini di tassi di migrazione tra classi di rating e loro stabilità, l'altro al processo di calibrazione ovvero alla fase di assegnazione delle PD. Il grado complessivo finale di ciclicità di un sistema di rating dipende da entrambi i processi.

In generale, i rating PIT sono associati a PD che riflettono le caratteristiche correnti della controparte, osservate con riferimento alle condizioni correnti del settore in cui opera e/o dell'economia nel suo complesso. Durante una fase di recessione, i rating PIT migrano verso classi di rating peggiori, pertanto nelle valutazioni PIT l'aumento della rischiosità del portafoglio si riflette in una migrazione di controparti dalle classi di rating migliori alle classi peggiori; di conseguenza, nel corso del tempo i tassi di default per una determinata classe di rating sono meno volatili mentre le matrici di migrazione sono più instabili. Al contrario, le valutazioni TTC sono (più) stabili nel tempo, non comportano una "reazione istantanea" di tali rating e quindi i tassi di default per una data classe di rating sono più volatili e le matrici di migrazione meno disperse. I modelli di rating comunemente utilizzati non corrispondono mai perfettamente alle tipologie "stilizzate" ed estreme sopra definite, ma si collocano in situazioni ibride.

I livelli di ciclicità del processo di attribuzione del rating e del processo di calibrazione condizionano dunque l'idoneità e l'accessibilità dei test di calibrazione, come quelli indicati dal Working Paper n.14 (Comitato di Basilea, 2005), di norma sotto l'ipotesi di indipendenza dei default. Infatti, essi sono concepiti per modelli tendenzialmente PIT e risultano quindi estremamente "penalizzanti" per modelli più vicini a una filosofia TTC. I problemi di idoneità si pongono in termini di coerenza "teorica" tra tipologia di test di calibrazione adottati e filosofia del sistema di rating; $i$ problemi di accessibilità si pongono in termini di probabilità di superare i test da parte di modelli di rating con livelli di ciclicità differenti.

Dunque, è possibile assumere diverse prospettive: come definire il processo di assegnazione del rating per superare $\mathrm{i}$ test di calibrazione? Come definire il processo di quantificazione delle PD per superare i test di calibrazione? Come sviluppare test di calibrazione che tengano in considerazione il livello di ciclicità del sistema di rating?

Teoricamente e in funzione delle scelte metodologiche effettuate, sia in termini di ordinamento delle controparti sia di calibrazione delle PD, è possibile costruire una matrice teorica di riferimento della filosofia di rating sottostante (PIT, TTC, sistemi ibridi).

Dall'analisi della normativa di Vigilanza di riferimento (Comitato di Basilea, CRR, Consultation Paper dell'EBA) è possibile ritrovare molti passaggi che trattano il tema della ciclicità, ma non sembra emergere un orientamento prevalente PIT/TTC in termini di assegnazione del rating, mentre sembra prevalere un indirizzo più spiccato per calibrazioni TTC che garantiscono una maggiore stabilità del requisito patrimoniale (indirizzo che purtroppo non trova sempre riscontro nelle verifiche ispettive di validazione, dove si chiede spesso ai modelli di rating di riflettere le condizioni economiche più recenti).

Anche la letteratura dedica molti paper al tema della calibrazione. Dopo aver esaminato questi contributi, nel corso del 2015, il gruppo di lavoro AIFIRM ha predisposto una survey per valutare le principali caratteristiche dei sistemi di rating correntemente usati dalle banche italiane, nonché le diverse applicazioni gestionali e le più opportune filosofie di rating adottate e i futuri sviluppi. Le banche aderenti sono state: Banca Popolare di Bari, Banca Popolare di Vicenza, Banco Popolare, BMW Bank, BPER, Cariparma, Che Banca!, Compass, Credem, Intesa Sanpaolo, Unicredit. Mentre per il Retail si è osservata una convergenza di opinioni sulla filosofia di rating attesa per i prossimi anni (maggiormente orientata al PIT), per i segmenti SME e Corporate le banche hanno una differente visione sul futuro orientamento dei sistemi di rating. Il legame controverso tra filosofia del sistema di rating e applicazioni gestionali, che si ritrova nelle risposte alla survey, è indicativo di un quadro concettuale e normativo incerto.

Il Position paper AIFIRM propone in conclusione 4 possibili direzioni da intraprendere per evitare che la simultanea coerenza con i requisiti normativi in materia di costruzione di modelli, in materia di calibrazione, i test suggeriti dal WP 14, le applicazioni gestionali e i requisiti di use test portino ad un insieme vuoto.

Direzione 1: il Regolatore definisca la misura del grado di ciclicità dei sistemi di rating e richieda dei test di calibrazione corretti per il ciclo. Le Banche sviluppano quindi modelli di rating con il grado di ciclicità desiderato, nel

${ }^{1}$ Aifirm ringrazia Silvio Cuneo e Giacomo De Laurentis (coordinatori del gruppo di lavoro) e Fabio Salis e Fiorella Salvucci 
rispetto dei requisiti regolamentari e di business, ed eseguono la convalida annuale utilizzando il framework di calibrazione corretto per il ciclo. Purtroppo, al momento il quadro metodologico non è ancora completamente consolidato e le soluzioni metodologiche individuate sembrano essere data-intensive rispetto alla lunghezza delle serie storiche a disposizione delle Banche.

Direzione 2: Il settore bancario di concerto con i Regolatori sviluppa un approccio ai test di convalida su più dimensioni: l'idea potrebbe essere quella di sviluppare una serie di test che valutino le prestazioni dei modelli e la loro conformità con i diversi (e spesso conflittuali) requisiti normativi. I nuovi test da introdurre, che si basano su diverse ipotesi di correlazione, ciclicità, etc... e che al momento purtroppo giocano un ruolo marginale nella discussione con $\mathrm{i}$ Regolatori, a causa anche della mancanza di una prassi condivisa e consolidata del settore bancario, potrebbero produrre come risultato una valutazione simultanea delle diverse dimensioni in un approccio a semaforo con soglie quantitative e giudizi qualitativi.

Direzione 3: il Regolatore definisca il target in termini di test di calibrazione e lasci che le Banche scelgano come allineare i processi di assegnazione e di calibrazione. Questa soluzione, pur avendo il vantaggio della chiarezza dei risultati, imporrebbe importanti vincoli sulle scelte metodologiche delle Banche, con il rischio di soluzioni non soddisfacenti per il business, a meno che non si rilassi il vincolo di use test e si conceda di utilizzare sistemi di rating diversi in funzione delle applicazioni gestionali.

Direzione 4: il Regolatore definisca interamente i requisiti in termini di processo di assegnazione del rating, processo di calibrazione delle PD, i test di calibrazione, lo use test. Tale approccio, che pare essere il più completo, risulta anche essere il più oneroso e complesso da definire

\section{The problem}

Often internal rating systems do not fully satisfy standard calibration tests used for PD validation.

According to the document "Studies on validation of internal rating systems" issued by the Basel Committee on Banking Supervision (WP n. 14, May 2005), still a milestone on this topic, "correct calibration of a rating system means that the PD estimates are accurate. Hence, for examining calibration somehow the differences of forecast PDs and realised default rates must be considered. This can be done simultaneously for all rating grades in a joint test or separately for each rating grade, depending on whether an overall assessment or an in detail examination is intended". 2 Many reasons could lead to fail calibration tests even by otherwise well performing rating systems. Two reasons are particularly critical: rating philosophy (TTC versus PIT ratings) and default correlation.

"The results of this analysis suggest that the pooled default probability assigned to each rating grade and its dynamics strongly depend on the type of rating system and the PD estimation method. The estimation from historical default rates is most meaningful when the pooled PDs are unstressed, which means that they are unbiased estimates of the likelihood of default in the following year. Furthermore, the analysis suggests that the long-run average default frequency for a through-the-cycle bucket will not provide a good approximation of that bucket's unstressed pooled PD. The reason is that the unstressed pooled PD will tend to be lower than the long-run average default frequency during cyclical peaks and higher than the long-run average default frequency during cyclical troughs". 3

"Due to correlation between defaults in a portfolio, observed default rates can systematically exceed the critical PD values if these are determined under the assumption of independence of the default events. This can happen easily for otherwise well-calibrated rating systems. As a consequence, on the one hand, all tests based on the independence assumption are rather conservative, with even well-behaved rating systems performing poorly in these tests. On the other hand, tests that take into account correlation between defaults will only allow the detection of relatively obvious cases of rating system miscalibration". 4

The WP 14 arrives at rather undetermined conclusions: "Therefore, statistical tests alone will be insufficient to adequately validate an internal rating system. Nevertheless, banks should be expected to use various quantitative validation techniques, as they are still valuable tools for detecting weaknesses in rating systems." "In conclusion, at present no really powerful tests of adequate calibration are currently available. Due to the correlation effects that have to be respected there even seems to be no way to develop such tests. Existing tests are rather conservative - such as the binomial test and the chi-square test - or will only detect the most obvious cases of miscalibration as in the case of the normal test. As long as validation of default probabilities per rating category is required, the traffic lights testing procedure appears to be a promising tool because it can be applied in nearly every situation that might occur in practice. ... Nevertheless, it should be emphasised that there is no methodology to fit all situations."

The WP 14 has assessed the quality of PD estimates by using methodologies based on the Binomial test, Chi-square test, Normal test, Traffic lights approach. These are still considered the "standard" reference framework for validating rating models' calibration.

\footnotetext{
${ }^{2}$ Studies on validation of internal rating systems, The Basel Committee on Banking Supervision, WP n. 14, May 2005, page 29.

${ }^{3}$ Idem, page 2.

${ }^{4}$ Idem, page 3 .

${ }^{5}$ Ibidem.

${ }^{6}$ Idem, page 34.
} 
A large number of scholars' and practitioners' papers are devoted to the issue of ratings calibration. After having examined these contributions, the AIFIRM work group on calibration has also prepared a questionnaire submitted to participant banks in order to assess current practices and future developments in the industry. The conclusions of the overall analyses and discussions have led to the proposal included in this position paper.

\section{Key question}

Supervisors and the industry are searching for new frameworks for validating rating models' calibration. The key question is: can calibration tests be discussed per se? The findings of WP 14 in year 2005 already suggested that the answer is no.

In fact, the impacts of rating philosophy and default correlations are many (Figure 1) and they also appear to be interconnected ${ }^{7}$.

Figure 1 Rating philosophy impacts.

\begin{tabular}{|l|c|c|c|c|c|c|}
\hline $\begin{array}{c}\text { Migration } \\
\text { rates }\end{array}$ & $\begin{array}{c}\text { Ratings } \\
\text { stability }\end{array}$ & $\begin{array}{c}\text { STD DEV of } \\
\text { forecasted } \\
\text { PDp }\end{array}$ & $\begin{array}{c}\text { Stability of } \\
\text { forecasted } \\
\text { PDp }\end{array}$ & $\begin{array}{c}\text { Observed DR } \\
\text { volatility per } \\
\text { rating class }\end{array}$ & $\begin{array}{c}\text { Asset or } \\
\text { default } \\
\text { correlation }\end{array}$ \\
\hline PIT & High & Low & High & Low & Low \\
\hline TTC & Low & High & Low & High & High & High \\
\hline
\end{tabular}

Legend: PDp is the portfolio probability of default.

\section{The role of the degree of pitness}

On one hand, the possibility that rating systems and estimated PD comply with standard calibration tests strongly depends on their degree of pitness. ${ }^{8}$ On the other hand, we still lack an universal definition of the degree of pitness of a rating system ${ }^{9}$.

In general, it is assumed that PIT ratings and/or associated PDs are focused on the current conditions of the borrower, observed in the current conditions of the business sector and/or of the economy as a whole. During a recession, PIT ratings migrate towards worse rating classes; therefore, in PIT ratings, the increase in the portfolio riskiness is reflected in a rating drift towards worse rating classes; hence, default rates for a given rating class are less volatile over time, that is in good and bad times. On the contrary, TTC ratings are (more) stable over time, and therefore default rates for a given rating class are more volatile (because in good and bad times the different portfolio default rates are factored in default rates volatility per rating class and not in ratings migrations).

However, a better understanding of the PIT/TTC ratings requires to distinguish two technically different concepts of degree of pitness: one is referred to ratings assignment processes, the other is referred to PDs calibration processes. The final overall degree of pitness of "the system" depends on both.

Many rating model building choices affect the "ratings degree of pitness" (in terms of migration rates, rating reversal rates,...), among which are the following:

1. Datasets time span (single-period, multi-period)

2. Observation period (the target time of prediction: $3 \mathrm{~m}, 1 \mathrm{y}, 2 \mathrm{y}, 3 \mathrm{y}, 5 \mathrm{y}$ )

3. Nature of independent variables (behavioral versus strategic)

4. Structure of independent variables (last data, averaged data)

\footnotetext{
${ }^{7}$ See Rosch. D. An empirical comparison of default risk forecasts from alternative credit rating philosophies, International Journal of Forecasting, 2005, page 47: "due to the lower asset correlation, the default distributions are in any case narrower under the Point in Time Rating scheme".

${ }^{8}$ We will use the terms rating philosophy, degree of pitness and degree of cyclicality of ratings as synonyms.

${ }^{9}$ Positions on this issue are still dispersed. See for instance Rosch. D., 2005, page 38: "an exact definition of a Point in Time Rating is possible - it reflects a borrower's 1-year probability of
} 
5. Perimeter of default definition (from past dues to filing bankruptcy petition)

6. Model estimation techniques and expert-judgment role.

Many calibration choices affect the "PDs degree of pitness" (in terms of closeness to actual DRs), among which are the following ways to associate PDs to ratings:
1. Downturn PDs
2. Last year DRs
3. Recent years DRs
4. Predicted PD central tendency

The degrees of pitness of rating assignment processes and rating quantification processes strongly impact on the suitability and accessibility of calibration tests (Figure 2) such as those indicated by the WP n.14 (the Binomial test, the Chi square test, the Normal test and the Traffic light approach). Suitability concerns the theoretical coherence of the test with the rating model philosophy. Accessibility concerns the probability to pass the test by rating models having different degrees of pitness.

Figure 2 Degree of pitness and suitability/accessibility of calibration tests.

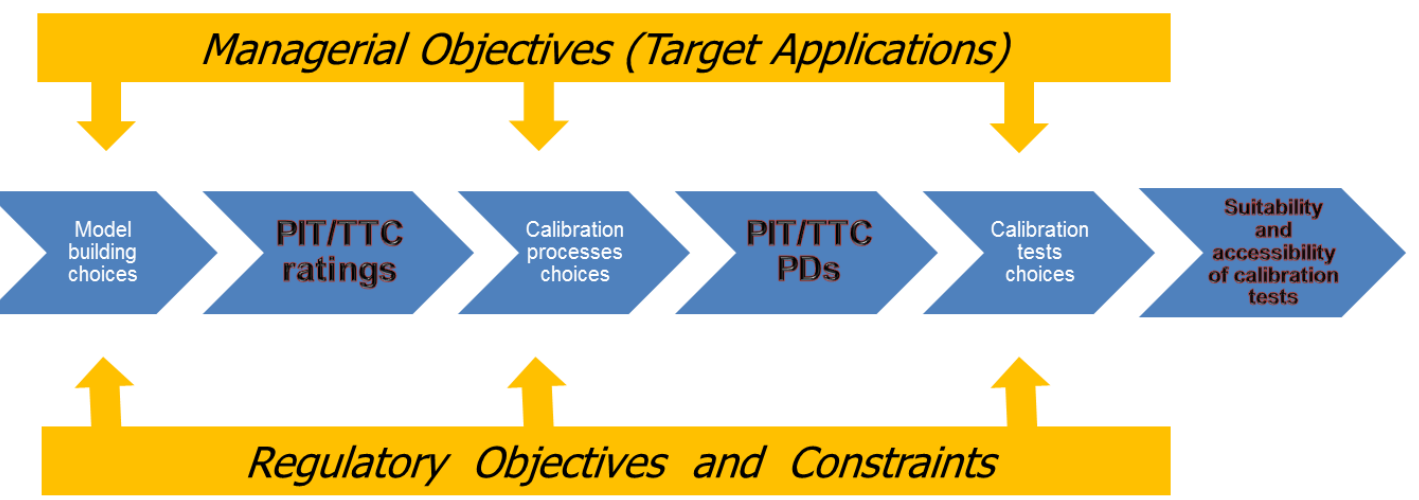

Therefore the following questions arise:

1. Have we to adjust rating assignment processes in order to meet calibration tests requirements?

2. Have we to adjust rating calibration processes in order to meet calibration tests requirements (whereas rating assignment processes are considered exogenous)?

3. Have we to adjust rating assignment processes and rating calibration processes in order to meet calibration tests requirements?

4. Have we to adjust calibration tests in order to take into account the degree of pitness of the rating system (that is considering rating assignment and calibration processes as exogenous)?

Theoretically we can have different possible combinations of choices related to rating assignment and calibration. Let's consider the four extreme cases (the four corners of Figure 3). Some choices, in either rating assignment or rating quantification processes cannot lead to pass current standard calibration tests.

The problem is twofold:

a) in order to pass calibration tests, specific choices on rating assignment and calibration are required,

b) some of the possible combinations are restricted by the regulation.

default-, while a definition of a Through the Cycle Rating is not as clear-cut". 


\begin{tabular}{|c|c|c|c|c|c|}
\hline \multicolumn{6}{|c|}{ Figure 3 Combinations of the degree of pitness of rating assignment and rating quantification processes. } \\
\hline & & \multicolumn{4}{|c|}{ Rating quantification processes } \\
\hline & & Pure TTC & TTC oriented & PIT oriented & Pure PIT \\
\hline \multirow{4}{*}{ 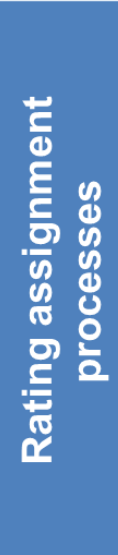 } & Pure TTC & $\begin{array}{c}\mathrm{PDp}=\mathrm{DR} \mathrm{R}_{\mathrm{LT}} \\
\text { No rating drift } \\
\text { Calibration tests failed } \\
\text { at } \mathrm{PDp} \text { level and at } \\
\text { each rating class level }\end{array}$ & & & $\begin{array}{c}\mathrm{PDp}=\mathrm{DR}_{\mathrm{BT}} \\
\text { No rating drift } \\
\text { Calibration tests } \\
\text { passed }\end{array}$ \\
\hline & TTC oriented & & & & \\
\hline & PIT oriented & & & & \\
\hline & Pure PIT & $\begin{array}{c}\mathrm{PDp}=\mathrm{DR} \mathrm{R}_{\mathrm{BT}} \\
\text { Strong rating drift } \\
\text { Calibration test passed }\end{array}$ & & & $\begin{array}{c}\text { Possible } \\
\text { overshooting in } \\
\text { PDp prediction } \\
\text { Calibration tests } \\
\text { may be failed }\end{array}$ \\
\hline
\end{tabular}

First of all, many paragraphs of Basel 2 regulation ${ }^{10}$ are influencing the PIT or TCC orientation of rating assignment and/or calibration processes (we use PIT/TTC combined with assignment/calibration after the symbol $>$ to give our view of the orientation implied in the rule):

a) 414. "Although the time horizon used in PD estimation is one year (as described in paragraph 447), banks are expected to use a longer time horizon in assigning ratings" $>$ TTC assignment

b) 415. "A borrower rating must represent the bank's assessment of the borrower's ability and willingness to contractually perform despite adverse economic conditions or the occurrence of unexpected events. For example, a bank may base rating assignments on specific, appropriate stress scenarios." $>$ TTC assignment

c) 444. "Internal ratings and default and loss estimates must play an essential role in the credit approval, risk management, internal capital allocations, and corporate governance functions of banks using the IRB approach" Undetermined, given that different applications may be better suited by systems with different degree of pitness

d) 447. "PD estimates must be a long-run average of one-year default rates for borrowers in the grade" calibration

e) 452. "A default is considered to have occurred with regard to a particular obligor when either or both of the two following events have taken place.... . The obligor is past due more than 90 days on any material credit obligation to the banking group" PIT assignment and calibration

f) 461. "Banks must use information and techniques that take appropriate account of the long-run experience when estimating the average PD for each rating grade" TTC calibration

g) 463. "Irrespective of whether a bank is using external, internal, or pooled data sources, or a combination of the three, for its PD estimation, the length of the underlying historical observation period used must be at least five years for at least one source. If the available observation period spans a longer period for any source, and this data are relevant and material, this longer period must be used." - TTC calibration

Let's consider now the CRR (Capital Requirement Regulation, EU 2013), art. 180, Requirements specific to PD estimation: "institutions shall estimate PDs by obligor grade from long run averages of one-year default rates" calibration.

Eventually, let's consider the Draft Regulatory Technical Standards On the specification of the assessment methodology for competent authorities regarding compliance of an institution with the requirements to use the IRB Approach in accordance with Articles 144(2), 173(3) and 180(3)(b) of Regulation (EU) No 575/2013, EBA Consultation Paper of 12

${ }^{10}$ Short for the document "International Convergence of Capital Measurement and Capital Standards. A Revised Framework, Basel Committee on Banking Supervision, June 2004". 
November 2014. At page 77 in the box with "text for consultation purposes": "the PD estimates should reflect the long run average of one-year default rates in order to ensure that they are relatively stable over time and extensive cyclicality of own funds requirements is avoided. It means that the PD estimates should be based on a period representative of the likely range of variability of default rates in that type of exposures in a complete economic cycle". TTC calibration.

In summary, the regulation tends to push towards a TTC orientation for rating quantification (calibration), whereas more contradictory requirements are set for rating assignment processes. Reconsidering now Figure 3 , we can conclude that none of the 4 extreme cases is at the same time:

- Compliant with regulatory requirements in terms of TTC rating assignment processes

- Compliant with regulatory requirements in terms of rating calibration processes based on a TTC approach

- Capable to pass calibration tests typically used in validation processes

In addition, a fourth perspective has not yet taken into consideration: the usefulness of "the system" for bank management processes. In fact, given the "use test" rule, the same rating system should be used for both regulatory and management purposes.

In conclusion, what we currently observe is:

a) an explicit TTC orientation of regulation for rating quantification processes,

b) a mixed PIT/TTC kinds of requirements in the regulation as far as rating assignment processes are concerned, and an (up to now) softer application by supervisors of TTC-oriented assignment processes regulatory requirements (this seems to be the case of supervisory validation processes for IRB banks).

As a consequence, the current situation is as in Figure 4, where red indicates systems not allowed by the regulation, olive indicates systems we are not yet capable to build (because of pure TTC or pure PIT assignment processes), green indicates what regulation wants (but standard calibration test will fail), and blue indicates what banks have done (but standard calibration test often fail for the insufficient degree of pitness of rating assignment processes).

Figure 4 An helicopter view of the situation.

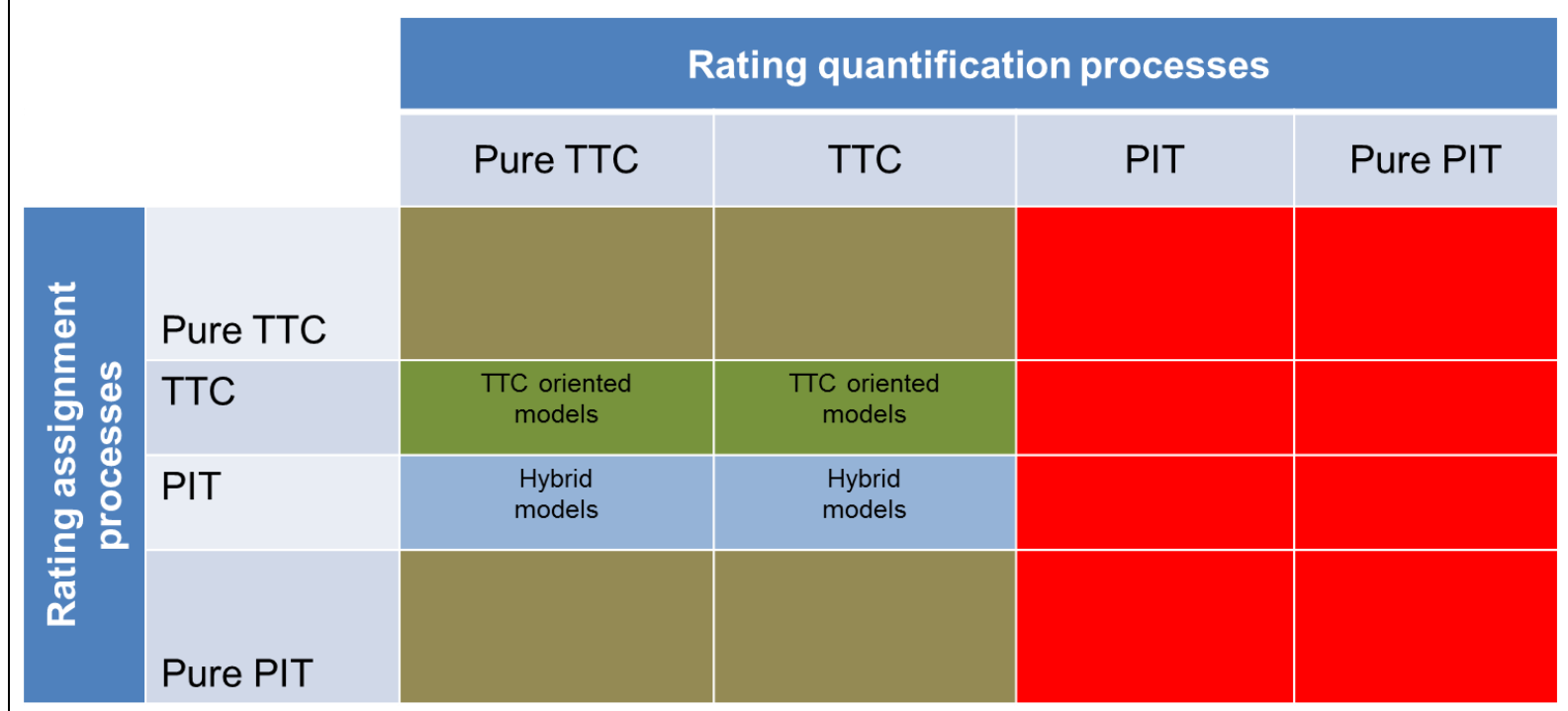

\section{Degree of pitness of rating assignment processes of current models}

In order to assess some relevant characteristics of rating systems currently used by Italian banks, the AIFIRM group on Validation of models' calibration has launched in 2015 a survey among its members. The following institutions has answered: Banca Popolare di Bari, Banca Popolare di Vicenza, Banco Popolare, Bmw Bank, Bper, Cariparma, Che Banca!, Compass, Credem, Intesa Sanpaolo, Unicredit.

What is the importance of different Information types in the model? The answers for rating models for corporate segments have outlined that financial statements, internal behavioural data and credit register data have a relevant role, whereas other qualitative pieces of information, both objective and subjective only have a minor role. In the case of 
models for SMEs, internal behavioural data and credit register data have an even more relevant role. In the case of models for the retail segment, the role of internal behavioural data and credit register data are of the utmost importance. See Figure 5.

Figure 5 Importance of different information types in rating assignment processes.

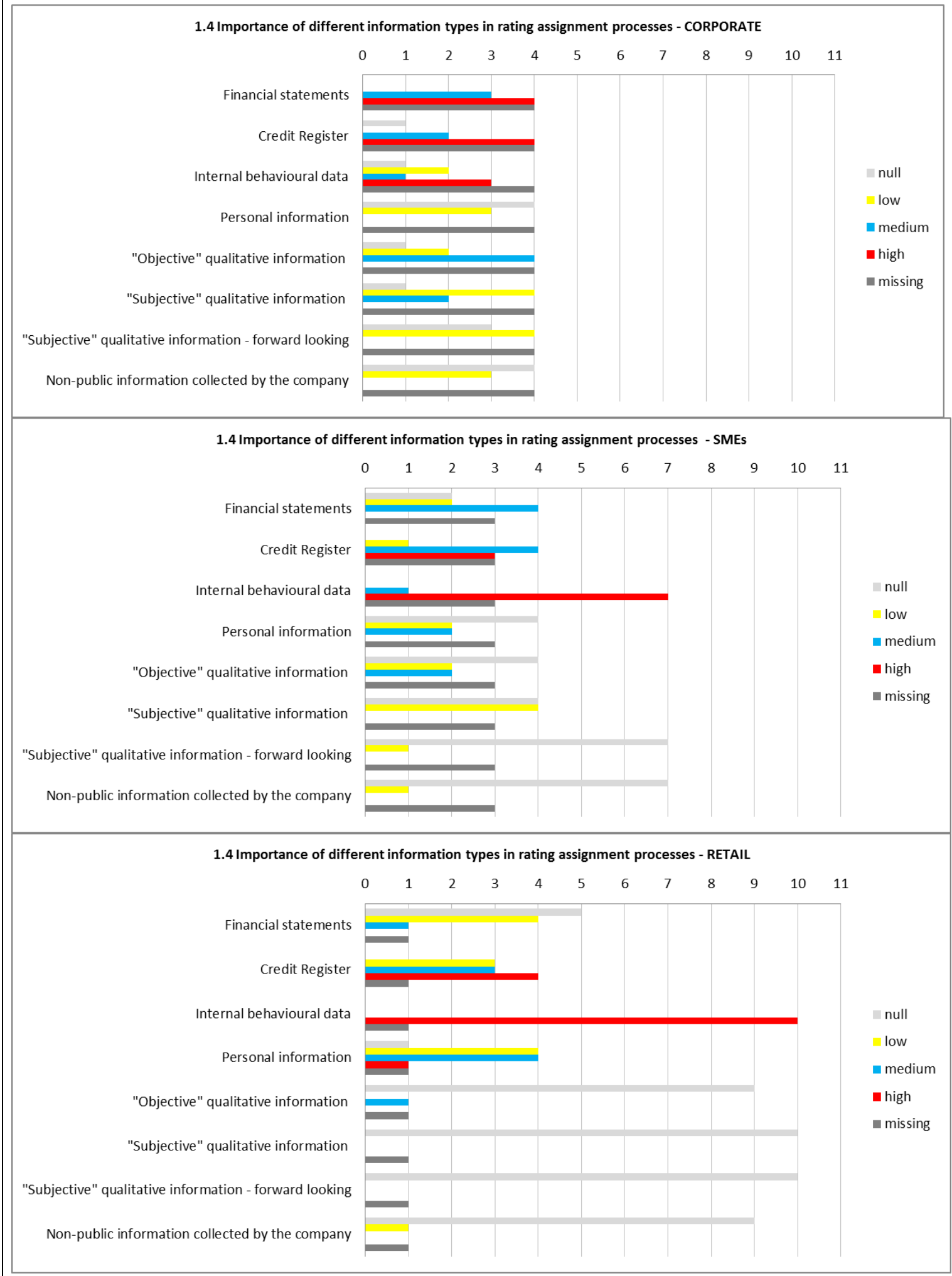


This qualitative perception of relative importance of different types of information is confirmed quantitatively by the ratio of the AuRoc of the individual module of the rating system (financial statement module, behavioral module, credit register module, "other information" module) and the final AuRoc of the system (Figure 6).

Figure 6 AuRoc ratios (individual module AuRoc divided by final model AuRoc.

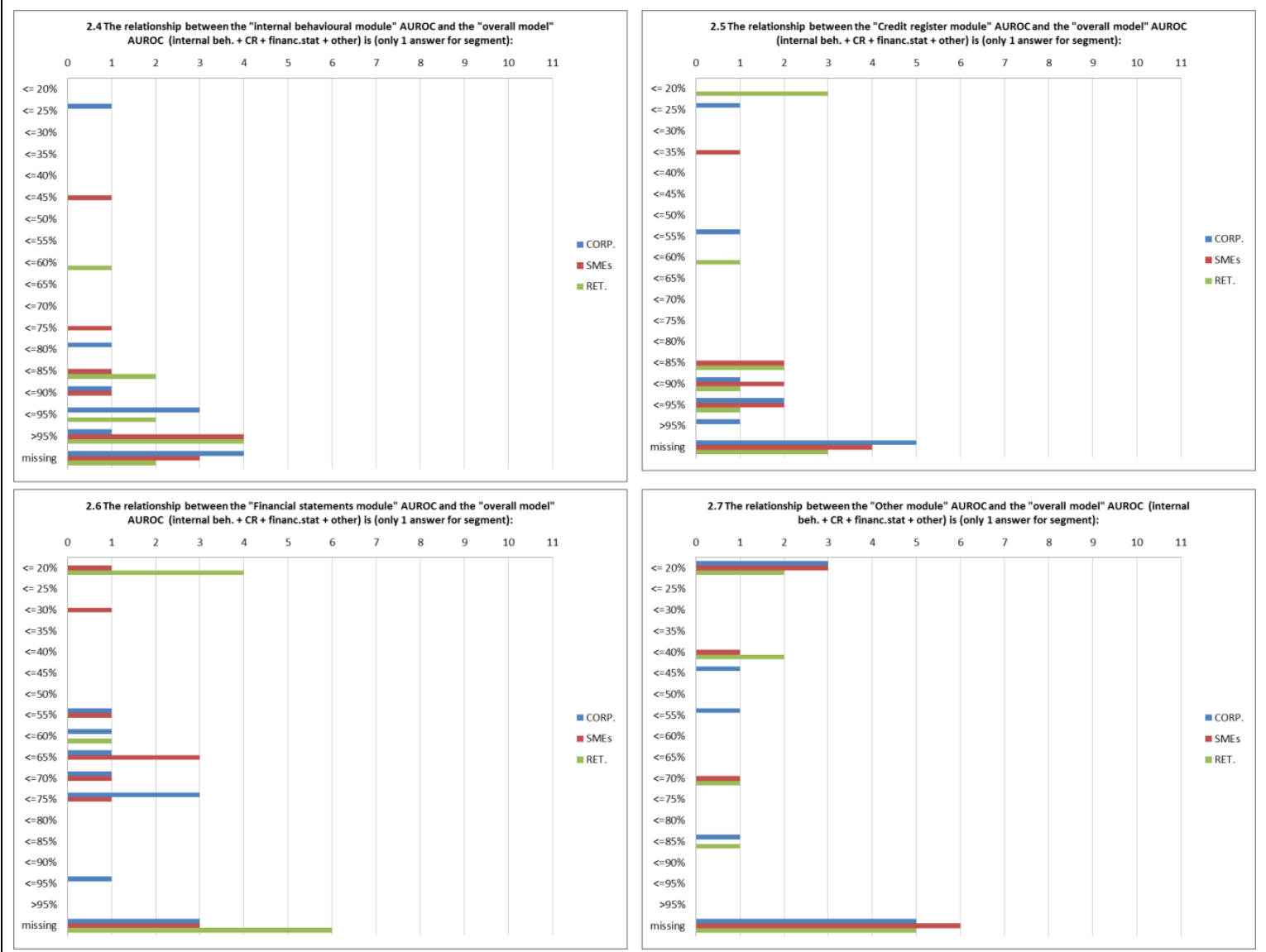

In practice, in banks that have communicated these ratios, the modules based on behavioral or credit register data are driving the final AuRoc of the model with little contribution of other sources of information.

Behavioral or credit register data have two important features:

a) they lead to PIT ratings as the assignment process focus on data that are timely pieces of information but whose predictive power is short-sighted. In other words, they lead to strong but short-term predictions. When the high frequency updating of these data is also considered, the end result is very high migration rates of assigned ratings;

b) they contribute aggressively to the final model scores and ratings when the observation period in model estimation is one-year; as soon as the observation period is set to a longer time horizon ${ }^{11}$, the estimation of the final model will attribute less relevance to this sort of data.

\section{Rating models' requirements for effective bank management}

In bank management it is evident that different ratings philosophies are desired for different types of applications .

\footnotetext{
${ }^{11}$ The observation period is the time length in which the default/non-default status of borrowers in the development dataset is observed. Let's remember here again that paragraph 414 of Basel 2 regulation requires it should be longer than one year: "Although the time horizon used in PD estimation is one year (as described in paragraph 447), banks are expected to use a longer time horizon in assigning ratings".
} 
Figure 7 Desired degree of pitness of rating systems for different applications.

\begin{tabular}{|l|c|}
\hline \multicolumn{1}{|c|}{ Rating uses } & $\begin{array}{l}\text { Sensitivity to } \\
\text { economic cycle }\end{array}$ \\
\cline { 2 - 3 } a. Underwriting (loan by loan) & High \\
\hline b. Customer relation & Low \\
\hline c. Commercial policy & Very high \\
\hline d. Early warning / watch list & High \\
\hline e. Risk control \& reporting & High \\
\hline f. Provisioning (current IAS) & Average \\
\hline g. Provisioning (IAS after G20 & High \\
\hline$\quad$ recommendations) & Average \\
\hline h. Economic capital & Low \\
\hline i. Regulatory capital (Pillar 1) & \\
\hline j. Capital adequacy (Pillar 2) &
\end{tabular}

Source: De Laurentis G., Maino R., Molteni L., Internal ratings, Wiley, 2010

According to the already mentioned AIFIRM survey, of the five large institutions that answered in full to the question, two banks do not see the need to differentiate rating system PIT/TTC orientation according to the specific applications whereas three banks do differentiate, but with some divergence of opinion (Figure 8).

Figure 8 Desired PIT/TTC orientation of future model developments for different applications

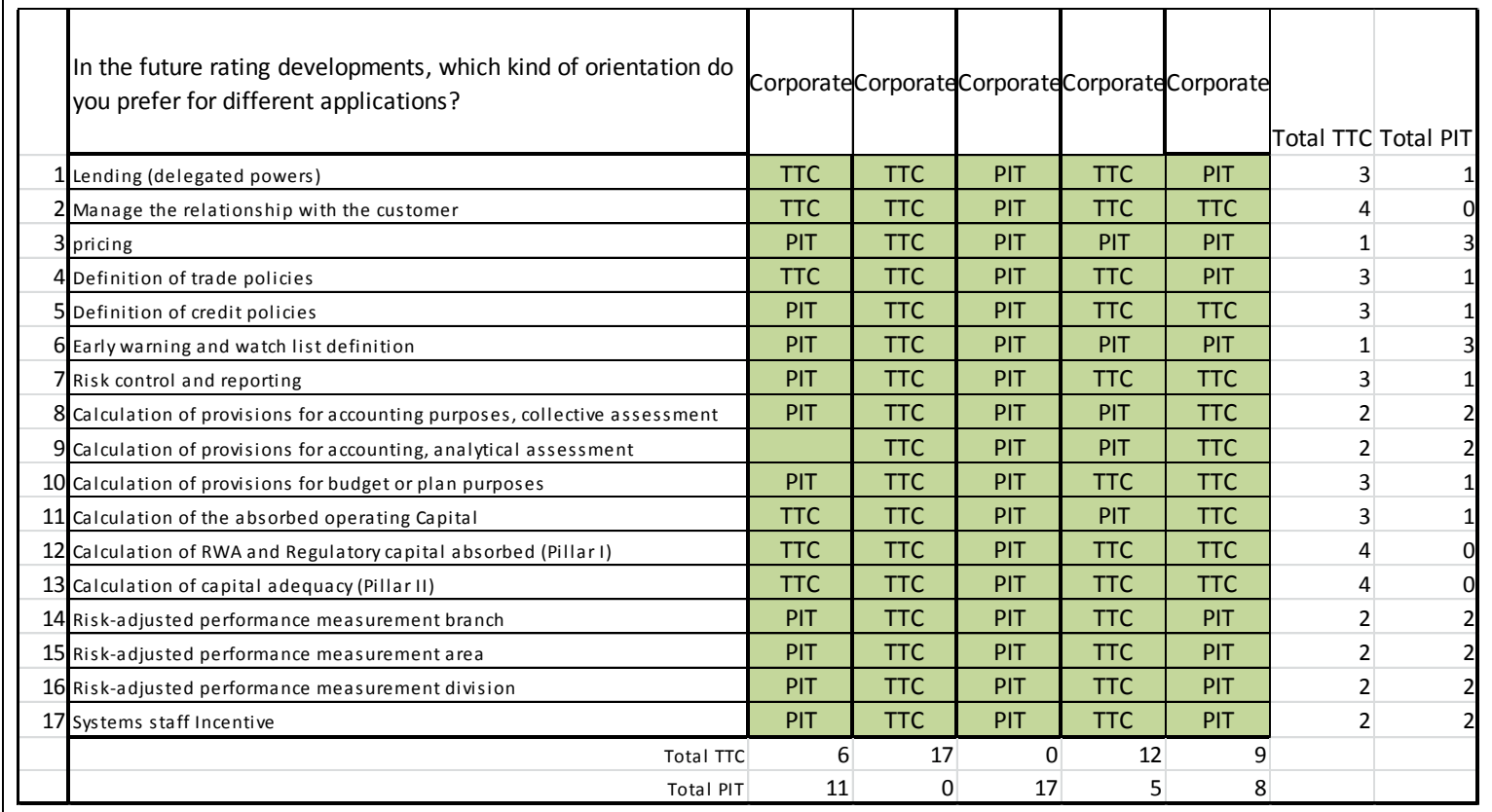

In general, banks are looking forward to having an higher degree of pitness for the retail segment, whereas in the SMEs and corporate segments different banks have different views on the future orientation of rating systems (Figure 9).

The controversial link between the rating philosophy and rating applications in the answers by banks surveyed by AIFIRM is indicative of an uncertain conceptual and regulatory framework. Also the "use test" requirements is possibly a source of this unclear view. It is worth to remember that paragraph 444 of Basel 2 states initially that "Internal ratings and default and loss estimates must play an essential role in the credit approval, risk management, internal capital allocations, and corporate governance functions of banks using the IRB approach", but immediately after it states that 
"It is recognised that banks will not necessarily be using exactly the same estimates for both IRB and all internal purposes. For example, pricing models are likely to use PDs and LGDs relevant to the life of the asset”.

Figure 9 Desired degree of pitness of future rating systems developments per applications and per segments

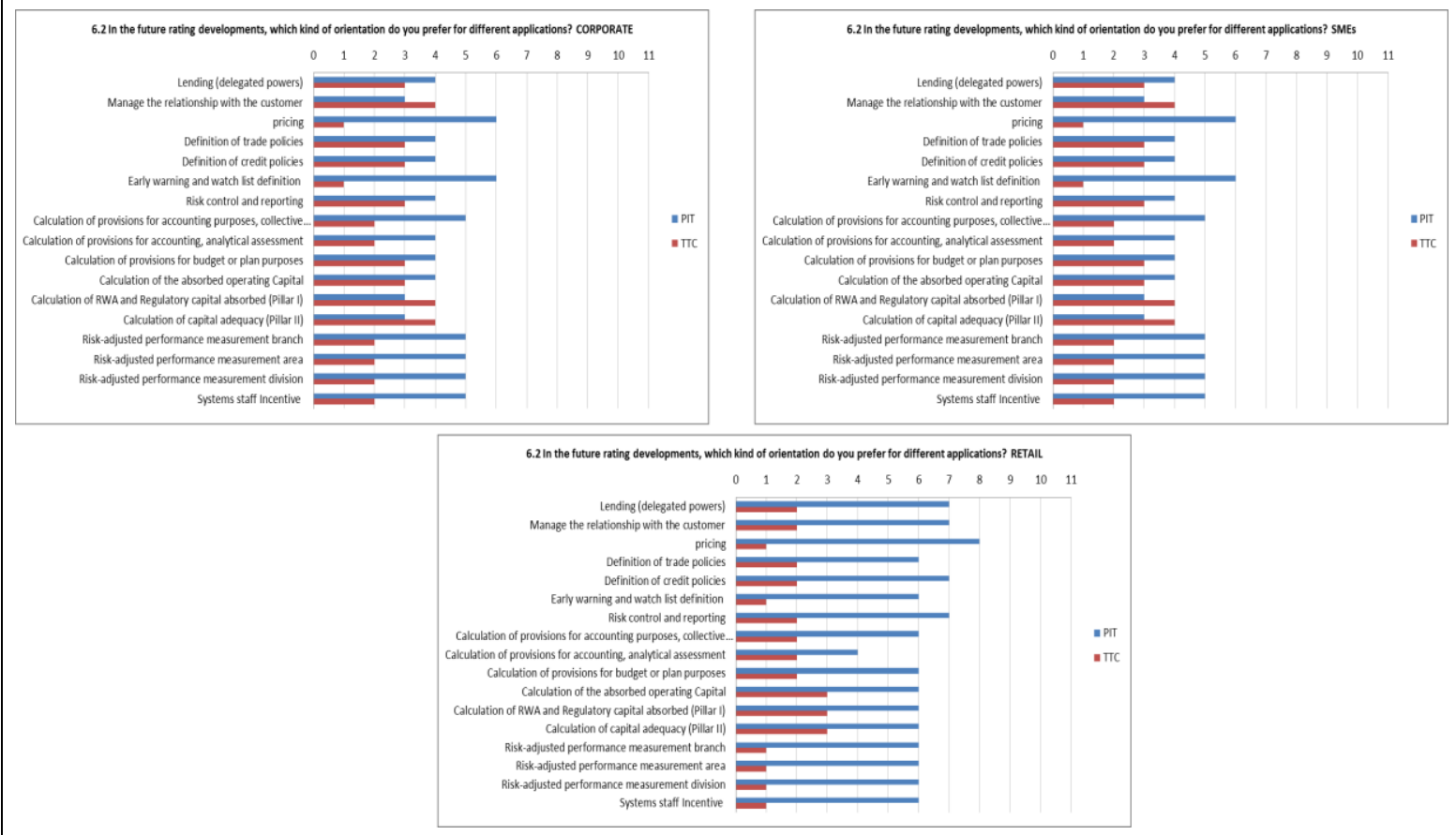

\section{Conclusions}

In conclusion, a simultaneous consideration of required compliancy with

a) regulatory requirements concerning model building (rating assignment processes)

b) regulatory requirements concerning model calibration (rating quantification processes)

c) standard calibration tests suggest by WP 14

d) the use test requirements

leads to an empty set.

Therefore, there are four possible directions to undertake.

Direction 1. Measure the degree of pitness of ratings (assignment and calibration processes) in order to set adequate calibration tests that take account of the degree of pitness of the system. This requires a universally accepted measure of degree of pitness and the development of pitness-sensitive calibration tests in order to allow for the rating cyclicality. This implies that:

a) the Regulators define an agreed degree of pitness measure to apply to Banks' rating models; AND

b) the calibration test(s) is conveniently modified in order to factor in the rating models degree of cyclicality;

AND

c) Banks develop the models with the desired degree of pitness but in compliance with all the other requirements discussed above and perform the annual validation using the pitness-adjusted calibration framework.

This solution preserves the flexibility in model development for Banks, thus ensuring the consistence between business and regulatory purposes and reduce the weight of alleged methodological inconsistency in the regulatory framework. 
Unfortunately, at the moment the methodological framework is not fully consolidated yet: there is not a common agreed degree of pitness measure and consistent tests set. Furthermore, available methodological solutions seem to be quite data intensive compared to the length of credit risk time series.

Direction 2. The industry agrees with Regulators a multidimensional approach to validation test. The idea is to develop a set of tests, each aiming at measuring the performance of the models and their compliance with different (even conflictual) regulatory requirements. Tests to be introduced are based on different hypotheses (correlations, degree of pitness measures, ...). At the moment these tests only play a marginal role in the discussion with Regulators (NCA, ECB, EBA), probably because of absence of an industry best practice. The outcome would be a simultaneous assessment of the several dimensions in a traffic light approach involving both quantitative thresholds and qualitative judgments. The traffic light approach could be implemented with specific threshold for each test, differentiated for the degree of pitness of the model, the target segment (corporate, retail, HDP, LDP,...), number of rating classes of the system,...

Direction 3. Use a calibration test level as a target and let banks choose how to align assignment and calibration processes to the target calibration results. This direction to sort out the conundrum requires to adapt the development process in order to produce rating models able to comply with the calibration test(s) that has been defined by the Regulator. This implies that:

a) The Regulators clearly define the calibration test(s) that the rating model must comply with. Note that this would obviously have some consequences on the rating model properties, namely on its degree of pitness;

b) The Banks accordingly develop the models and perform the annual validation.

It can be argued that this solution, while having the pro of clarity, would impose serious constraints on the methodological choices. Banks may end up with models non satisfactory for their business purposes, unless the use test requirement is relaxed and the use of different rating systems for different purposes are allowed. Of course, the analysis of coherence of different rating systems becomes a part of the validation process.

Direction 4. Propose a new set up of regulation in order to get a better alignment of requirements in terms of rating assignment processes, PD calibration processes, calibration tests, use test. This solution leaves on regulators the entire burden of re-thinking assignment and calibration requirements, use test requirements and calibration tests framework setting. It could be the more comprehensive approach but also the most difficult and time expensive solution.

Silvio Cuneo, Giacomo De Laurentis, Fabio Salis e Fiorella Salvucci

Sono disponibili sul sito www.aifirm.it l'Allegato 1 contenente l'analisi della letteratura realizzata da Valeria Stefanelli e l'Allegato 2 contenente il questionario di indagine realizzato anche con il prezioso contributo di Marco Salemi 


\section{References}

- $\quad$ Aguais S. (2008), Designing and Implementing a Basel II Compliant PIT-TTC Ratings Framework, MPRA Paper No. 7004, posted 6. February 2008

- Alfonsi D. (2010), Informal Expert Working Group on Rating backtesting in a cyclical context Methodological Annex, Main findings and proposals, slides CEBS Consultative panel

- Altman E.I, and A. Rijken H. A. (2006), A Point-in-Time Perspective on Through-the-Cycie Ratings, Financial Analysts Journal, Vol. 62, n.1

- Blochlinger A., Leippold M. (2005), Testing probability calibration validation: application to credit scoring models, PAPER

- Boegelein L. (2005), Validation of Internal Rating and Scoring Models, Global Financial Services Risk Management, Ernst \& Young

- Breinlinger L., Glogova E. and Hoger A. (2003), - Blochlinger A., Leippold M. (2005), Calibrations of rating system: A first analysis, PAPER

- Carlehed M. and Petrov A. (2012), A methodology for point-in-time-through-the-cycle probability of default decomposition in risk classification systems, Journal of Risk Model Validation, Vol. 6, n. 3, Fall 2012

- Castermans G., Martens D., Gestel T. V., Hamers B. and Baesens B. (2007), An Overview and Framework for PD Backtesting and Benchmarking, paper

- Cesaroni T. (2015), Prociclicality of credit rating systems: how to manage it, Working Papers, Banca d'Italia, September 2015

- Cornaglia A. (2010), Ciclicità dei rating e stress test 1. Filosofia e ciclicità nei sistemi di rating, presentazione, Finance Master Class Milano

- Cornaglia A. and Morone M. (2009), Rating philosophy and dynamic properties of internal rating systems: A general framework and an application to backtesting, MPRA Paper No. 14711, posted 19 April 2009

- $\quad$ EBA (2014), On the specification of the assessment methodology for competent authorities regarding compliance of an institution with the requirements to use the IRB Approach in accordance with Articles 144(2), 173(3) and 180(3)(b) of Regulation (EU) No 575/2013, EBA/CP/2014/36 (published November 12 2014)

- Engelmann B. and Rauhmeier R. (2006), Chapter 14: Statistical approaches to PD validation, in, The Basel II risk parameters: Estimation, Validation, Stress Testing with Applications to Loan Risk Management, Springer, II Edition:

- Fei F., Fuertes A. and Kalotychou E. (2011), Credit Rating Migration Risk and Business Cycles, Journal of Business Finance and Accounting (forthcoming).

- Gobeljic P. (2012), Classification of Probability of Default and Rating Philosophies, paper

- Kauko K. (2010), The feasibility of through-the-cycle ratings, paper

- Kauko K. (2012), Can Credit Risk Be Rated Through-the-Cycle?, Frontiers in Finance and Economics, Vol. 9 N ${ }^{\circ}$, pp. 132

- $\quad$ Kiff J., Kisser M. and Schumacher L. (2013), Rating Through-the-Cycle: What does the Concept Imply for Rating Stability and Accuracy?, IMF Working Paper, WP/13/64

- Moody's, Complementing Agency Credit Ratings with MIR® (Market Implied Ratings), Moody’s Analytics Credit research e risk measurement, http://www.moodysanalytics.com

- Petrov A. (2012), Implementing pit-ttc pd framework in credit risk classification system, presentazione Risk Minds 2012 Amsterdam, (slides)

- $\quad$ Rikkers F., Thibeault A. (2008), The influence of rating philosophy on regulatory capital and procyclicality, paper

- Rosch D. (2005), An empirical comparison of default risk forecasts from alternative credit rating philosophies, International Journal of Forecasting, Vol. 21, pp.37-51

- Standard \& Poor's (2014), Default, Transition, and Recovery: 2013 Annual Global Corporate Default Study And Rating Transitions, Standard\&Poor's Ratings Services, McGraw Hill Financial

- Topp R. and Perl R. (2010), Through-the-Cycle Ratings Versus Point-in-Time Ratings and Implications of the Mapping Between Both Rating Types, New York University Salomon Center and Wiley Periodicals

- Vallés V. (2006), Stability of a "through-the- cycle" rating system during a financial crisis, Financial Stability Institute, BIS, Award 2006 Winning Paper 


\section{Stress test e Rischio sistemico}

\section{Positon Papaer di Aifirm ${ }^{1}$}

\section{Abstract}

The recent financial crisis has showed the weakness and the lack of prudential supervision instruments to prevent a systemic crisis. The overall framework of Supervision seemed to be inadequate to take up any control actions concerning supranational banks with different regulations or supervision practices. The weakness of internal bank governance, distorted incentives, the lack of macro-prudential supervision have facilitated and amplified the economic crisis. The strong connections among economics and financial institutions have allowed the crisis to spread out with a significant increase of systemic risk.

To ensure a right managing of the systemic risk, new supervision instruments were introduced by the Institutions. This contribution is focused on the response made by the Institutions. This analysis shows how the general response to manage a systemic risk is based on the assumption "more capital, less risk". This assumption couldn't be the right way to reduce the systemic risk considering the existing trade-off between profitability and capital and liquidity adequacy. Capital requirements represents a strong defense against risks, but overcapitalization doesn't seem to be another defense; consequently the impossibility to reach adequate capital remuneration could determinate a general deleveraging or a reallocation toward higher risk assets with a relevant impact on the economy and consequently on the systemic risk.

\section{Introduzione}

La recente crisi finanziaria ha evidenziato come la Vigilanza Prudenziale e gli strumenti a disposizione dei Supervisori non fossero adatti a fronteggiare le crisi sistemiche. Il modello di Vigilanza pre-esistente, incentrato sulle Autorità Nazionali, si è rilevato inadeguato ad intraprendere le necessarie azioni di controllo, anche in considerazione della nascita di imprese bancarie sovra nazionali operanti in più Stati e soggette a regolamentazioni o prassi di vigilanza spesso molto diverse tra loro. I dibattiti successivi sulle regole e sulla struttura di vigilanza hanno portato ad un indebolimento delle Autorità Nazionali con cessioni di sovranità dagli Stati nazionali a strutture sovra nazionali. Congiuntamente si è assistito ad un ampio processo di armonizzazione e rafforzamento delle regole di Vigilanza prudenziale e alla nascita di ulteriori strumenti.

Le nuove regole di Vigilanza hanno posto particolare enfasi sul rischio sistemico ovvero "il rischio che il fallimento di un partecipante nell'adempiere ai suoi obblighi contrattuali possa a sua volta causare il fallimento di altri partecipanti", ampliando fortemente la gamma di strumenti a disposizione dei Supervisori per mitigare tale rischio. In linea di massima è possibile classificare gli strumenti di Vigilanza in:

1. misure volte alla riduzione della probabilità che si verifichi un evento sistemico in forma forte, quindi inteso come riduzione della probabilità di fallimento di una banca che potenzialmente può generale un effetto contagio;

2. misure finalizzate a rafforzare la capacità della singola banca di resistere a un evento sistemico, di fatto per farsi che tale evento sia in forma debole e non in forma forte e, pertanto, maggiormente fronteggiabile.

Possiamo quindi individuare misure che riguardano soltanto le banche che possono originare un evento sistemico, e misure che riguardano tutte le banche e che sono finalizzate a rafforzarne le capacità di resilienza nel caso in cui l'evento sistemico si verifichi.

Tra le misure classificabili tra quelle finalizzate alla riduzione della probabilità che si verifichi un evento sistemico è possibile annoverare: il TLAC ed il MREL, il G-SIIBs buffer, ed i piani di recovery e resolution. Tutte le altre misure, ivi inclusi i requisiti di capitale, possono essere annoverate nella seconda categoria.

Alla base delle scelte adottate dai Regolatori, è tuttavia possibile affermare che il criterio che ha maggiormente inciso nella selezione degli strumenti è stato l'assioma "maggior patrimonio implica minor rischio".

I recenti fallimenti bancari, allorché avvenuti con le vecchie regole di vigilanza prudenziale "Basilea II", hanno dimostrato che non sempre il patrimonio rappresenti una garanzia di minor rischiosità dell'Ente. I livelli di patrimonializzazione di molte delle banche entrate in dissesto o fallite nel corso della recente crisi finanziaria erano tutt'altro che contenuti, spesso notevolmente al di sopra della media del settore e dei minimi regolamentari.

Il position paper ha come scopo iniziale quello di definire il rischio sistemico nel quadro regolamentare attuale (primo e secondo capitolo).

Nel terzo capitolo provvederemo ad enuclearne i differenti strumenti di vigilanza prudenziali finalizzati alla mitigazione del rischio sistemico. Come si vedrà, gli strumenti predisposti dai Regulators sono estremamente variegati e spesso non direttamente esplicitati nella normativa di riferimento. Sono soggetti ad ampie discrezionalità a livello di applicazione e non esiste attualmente un criterio di priorità nella selezione degli strumenti. Come si vedrà il criterio guida risulta essere quello di dotare le Banche di requisiti patrimoniali sempre più onerosi.

Nel quarto capitolo vedremo come le dotazioni patrimoniali risultino ad oggi ampiamente sovrastimate rispetto ai rischi verso i quali gli Enti sono effettivamente esposti. Il focus di intervento è prevalentemente sul rischio di credito. Si dimostrerà quantitativamente che le correlazioni delle funzioni di ponderazioni risultano sovrastimate rispetto all'effettivo livello di

\footnotetext{
1 Aifirm Ringrazia: Fabio Salis (Banco Popolare) - Coordinatore della Commissione; Pietro Penza (PWC) - Coordinatore della Commissione; Pasquale Costa (Banco Popolare); Nicola Mariano (PWC); Ester Inserra Imparato (Cariparma - Credit Agricolé); Ruggiero Gorgoglione (Ernst \& Young); Francesco D'Avanzo (Cariparma - Credit Agricolé); Camillo Gilberto (MPS); Marco Rocchi (Cariparma Credit Agricolé); Andrea Consonni (Intesa San Paolo); Agostino Grua (Banca Popolare di Bari); Francesco Mazza (Credito Valtellinese); Claudio Barberi (Banco Popolare); Antonio Castagna (Iason); Giorgio Canobbio (Concentric); Marco Salemi (Banca del Mezzogiorno Medio Credito Centrale);
} 
rischio generando così assorbimenti patrimoniali eccessivi. L'eccesso di requisito patrimoniale può, pertanto, già essere inteso come quell'add-on richiesto alle banche come "garanzia" nei confronti del sistema per mitigare il rischio di fallimento dell'ente. Per gli altri rischi si forniranno delle evidenze qualitative finalizzate a dimostrare l'esistenza di un double counting del rischio e, pertanto, di un ulteriore add-on di capitale già disponibile a copertura del rischio sistemico.

\section{Premessa}

E' ormai dal lontano 2007, anno in cui la crisi dei mutui sub-prime americani ha generato lo tsunami che ha investito il sistema bancario e le economie di mezzo mondo, che ci si pone la stessa domanda: perché l'accordo di Basilea II non è stato in grado di evitare la crisi? Perché nonostante le regole, nonostante le evoluzioni dei protocolli di capitalizzazione delle Banche non si è stati in grado di evitare il collasso di molti istituti e i rilevanti costi ricaduti sull'economia e sul sistema finanziario pubblico e privato?

La risposta è sicuramente complessa, come è complesso individuare un unico punto in cui il meccanismo si è inceppato. Bisogna comunque tener conto che, probabilmente, l'esistenza del protocollo e delle misure di capitalizzazione ha evitato un disastro di proporzioni molto maggiori di quanto si sia verificato.

Per semplicità è possibile suddividerle le cause della crisi finanziaria in due categorie, ossia cause microeconomiche e cause macroeconomiche.

Le cause microeconomiche potrebbero a loro volta essere classificate nei seguenti ambiti: distorsione degli incentivi; problemi di misurazione e gestione del rischio; lacune nella regolamentazione e nella vigilanza.

Le cause macroeconomiche potrebbero essere ascritte alle seguenti due categorie: problemi legati all'accumulo di squilibri nelle posizioni creditorie internazionali e difficoltà create dal prolungato periodo di bassi tassi di interesse reali.

Le cause della crisi sono prioritariamente da rintracciare nell'andamento dell'economia nei primi anni del 2000, caratterizzata da bassi tassi di interesse ed un abbondante liquidità a livello di sistema. Questo contesto economico ha favorito negli Stati Uniti d'America una eccessiva crescita del credito, nonché del valore reale degli immobili. A sua volta, le garanzie reali (che aumentavano) riducendo il loan to value degli immobili posti a garanzia contribuivano a rafforzare l'offerta di credito. I bassi rendimenti conseguenti l'elevata liquidità ha spinto gli operatori di mercato ha ricercare opportunità che hanno conseguentemente rafforzato l'offerta di strumenti molto complessi, difficilmente valutabili e caratterizzati da una eccessiva leva finanziaria. Questi prodotti hanno rappresentato lo strumento per il contagio e la propagazione dei rischi una volta che il rafforzarsi dell'economia e delle aspettative di inflazione ha determinato un incremento del costo del denaro. Tassi di interesse elevati hanno determinato una significativa riduzione della liquidità ed un calo generalizzato del valore degli immobili e conseguentemente una contrazione del credito; hanno inoltre indebolito la capacità di rimborso del credito ottenuto da parte dei clienti. Le maggiori sofferenze bancarie hanno infine causato un crollo del valore dei prodotti finanziari che avevano come sottostante proprio gli asset creditizi.

Quanto accaduto è stato certamente possibile anche grazie ad una errata comprensione dell'interazione tra rischio di credito e rischio di liquidità, nonché dall'omessa verifica della leva finanziaria degli istituti di credito. La gestione del rischio ha certamente rappresentato uno degli aspetti maggiormente critici, spesso caratterizzato dalla presunzione del mercato di valutare correttamente i rischi assunti nonostante la mancanza di regolamentazione di interi comparti (c.d. sistema bancario ombra) e l'assenza di trasparenza e comparabilità tra Istituti di credito spesso caratterizzati da regolamentazioni troppo poco omogenee e/o da discrezionalità nazionali troppo eterogenee tali da non garantire una reale armonizzazione agli standard di Basilea.

Un altro punto che ha giocato a sfavore del protocollo di Basilea II è stata la pesante introduzione dell'utilizzo di rating esterni per la definizione della rischiosità delle controparti, in particolare per quelle appartenenti ai settori bancari. Tale pratica ha, di fatto, consegnato la possibilità degli Istituti di rifornirsi di liquidità sul mercato interbancario alle decisioni della agenzie di rating. Tale problematica si è manifestata durante la crisi (dal 2007 in avanti) quando le ripetute revisioni al ribasso da parte delle agenzie di rating internazionali hanno causato una serie di anomalie di liquidità sul mercato dei capitali ampliando gli effetti della crisi finanziaria. Da questo punto di vista il ricorso ai rating esterni ha rappresentato sicuramente un fattore di debolezza di Basilea II non consentendo alle Banche, nel momento di crisi, di approvvigionarsi a costi ragionevoli di liquidità sul mercato interbancario e favorendo ancora di più il "credit crunch".

Gli errori i valutazione sono stati inoltre favoriti da un sistema di Governance Societaria troppo incentrato su aspetti legati alla sola redditività degli investimenti. La carenza dei sistemi di controllo era favorita anche da policy di remunerazione che incentivavano l'assunzione dei rischi, con investimenti mirati a garantire la redditività nel breve periodo a discapito della sostenibilità del business nel medio e lungo termine.

In questo contesto le politiche e le pratiche di vigilanza sono state carenti nella prevenzione e nella gestione della crisi. Emblematica è l'insufficiente o assente attenzione accordata al rischio di liquidità, o l'eccessiva fiducia riposta nelle Agenzie di Rating, o la sottostima dei requisiti patrimoniali sui modelli interni spesso determinata da prassi di Vigilanza molto diverse tra i Paesi membri.

Tra tutto questo, probabilmente, le maggiori carenze regolamentari sono da imputare all'assenza di un quadro di vigilanza macroprudenziale, in grado di valutare i rischi sistemici, di prevenirli e gestirli dotando le Istituzioni di strumenti efficaci ed efficienti che prevengano l'accumulo di squilibri finanziari e la probabilità di contagio tra gli Istituti ed il sistema a causa delle pesanti interconnessioni presenti tra le Banche, il mercato dei capitali e quello dei titoli finanziari e dei derivati. 


\section{Rischio Sistemico e crisi del sistema bancario}

\subsection{Vigilanza Micro e Macro Prudenziale e il Rischio Sistemico}

Uno dei principali insegnamenti della recente crisi finanziaria è che le politiche di regolamentazione devono avere un più spiccato orientamento macroprudenziale per affrontare in modo compiuto i rischi finanziari sistemici.

Le prima definizione di politica macroprudenziale risale al Comitato Cooke, precursore dell'attuale Comitato di Basilea, che avrebbe coniato tale termine nel $1979^{2}$. Il termine oggi è ampiamente utilizzato per definire genericamente un plateau di misure e strumenti che abbracciano diversi ambiti (politiche monetarie, fiscali e valutarie) finalizzate anche alla stabilità finanziaria. La riserva obbligatoria o le operazioni di LTRO possono essere intese come strumento di politica macroprudenziale allorché consentano di mitigare il rischio di liquidità.

In questo ambito, è utile ascrivere l'oggetto dell'analisi a quel set di strumenti che hanno come obiettivo primario esplicito la stabilità del sistema finanziario nel suo complesso, ovvero gli strumenti di vigilanza prudenziale.

Essenzialmente la politica macroprudenziale amplia la prospettiva della politica prudenziale tradizionale integrando e rafforzandone gli strumenti a disposizione. E' immediato comprendere lo stretto legale tra la vigilanza micro e quella macroprudenziale. Il pieno conseguimento della prima è di per se la principale garanzia per la stabilità finanziaria nel suo complesso.

Probabilmente volendo categorizzare gli strumenti di vigilanza al fine di ricondurli nell'una o nell'altra categoria è necessario riferirsi all'obiettivo cui lo strumento dovrebbe riferirsi, pertanto:

- gli strumenti di vigilanza microprudenziale garantiscono la solvibilità e la liquidità dei singoli intermediari finanziari, ovvero sono finalizzati ad assicurare la sana e prudente gestione mediante l'adozione di prassi ottimali di vigilanza, limitando l'assunzione di rischi da parte degli intermediari e rafforzandone le dotazioni patrimoniali;

- gli strumenti di vigilanza macroprudenziale mirano a garantire la stabilità del sistema finanziario nel suo complesso rafforzandone la resilienza mediante una riduzione chiara e realizzabile del rischio sistemico.

La sana e prudente gestione dei singoli intermediari e la stabilità del sistema finanziario nel suo complesso sono obiettivi complementari finalizzati a prevenire il fallimento di uno o più intermediari, sia a causa dell'esposizione verso rischi comuni (es. eccessiva crescita dei valori immobiliari), sia perché uno di essi ha dimensioni o interconnessioni tali da determinare fallimenti bancari a catena e conseguentemente un dissesto di portata sistemica.

L'analisi macroprudenziale deve, pertanto, individuare tutti quei fattori di rischio e quelle vulnerabilità cui il sistema finanziario nel suo complesso è esposto o potrebbe essere esposto al fine di prevenire la formazione di minacce i cui effetti, in ultima istanza, andrebbero ad intaccare l'economia reale. L'analisi macro prudenziale deve prestare particolare attenzione ai fattori di rischio comuni o correlati e soprattutto a quei istituti di credito che, a causa della loro "rilevanza sistemica", potrebbero causare o amplificare un rischio contagio. La vigilanza macroprudenziale, conseguentemente, consiste in un insieme di attività, analisi, segnalazioni e raccomandazioni, regolamenti e ispezioni il cui scopo è mitigare il rischio sistemico.

\subsection{Governo del Rischio Sistemico - La nuova Governance di Vigilanza}

A seguito della crisi finanziaria, è apparso chiaro come sia le regole di vigilanza prudenziale sia i meccanismi di vigilanza non fossero più adatti a fronteggiare un mercato finanziario sempre più complesso ed integrato. Inoltre, la struttura della Vigilanza non prevedeva specifici strumenti di Vigilanza Macroprudenziale con Regolatori e Supervisori.

Come noto, l'approccio adottato è stato guidato dalla volontà di integrare maggiormente l'Unione europea, portando alla realizzazione di una vera e propria Banking Union. La Governance di vigilanza ha previsto maggiori poteri delegati dagli stati membri alle autorità sovranazionali, il rafforzamento degli ex comitati di terzo livello in vere e proprie Agenzie, la creazione del Consiglio europeo per il rischio sistemico e l'istituzione dell'European System of Financial Supervision.

Il Sistema Europeo di Vigilanza Finanziaria (ESFS) è stato creato al fine di garantire una vigilanza finanziaria armonizzata ed integrata a livello di Unione Europea garantendo così reali condizioni di parità per tutti gli attori e rispecchiando l'integrazione sempre maggiore dei mercati finanziari europei.

Questo in ultima analisi, ha portato alla creazione di un sistema di vigilanza micro e macroprudenziale composto da autorità di vigilanza europee e nazionali. Il pilastro microprudenziale a livello europeo è formato dall'Autorità bancaria europea (EBA), dall'Autorità europea degli strumenti finanziari e dei mercati (ESMA) e dall'Autorità europea delle assicurazioni e delle pensioni aziendali e professionali (EIOPA), che insieme al Comitato congiunto delle autorità europee di vigilanza (Joint Committee) formano l'Autorità di vigilanza Europea (ESA). La vigilanza macroprudenziale è effettuata dal Comitato europeo per il rischio sistemico (ESRB). Le autorità di vigilanza nazionali competenti (NCA) dei rispettivi Stati membri sono anch'esse parte dell'ESFS.

${ }^{2}$ Origini ed evoluzione del termine macroprudenziale - Rassegna stampa BRI - P. Clement. 


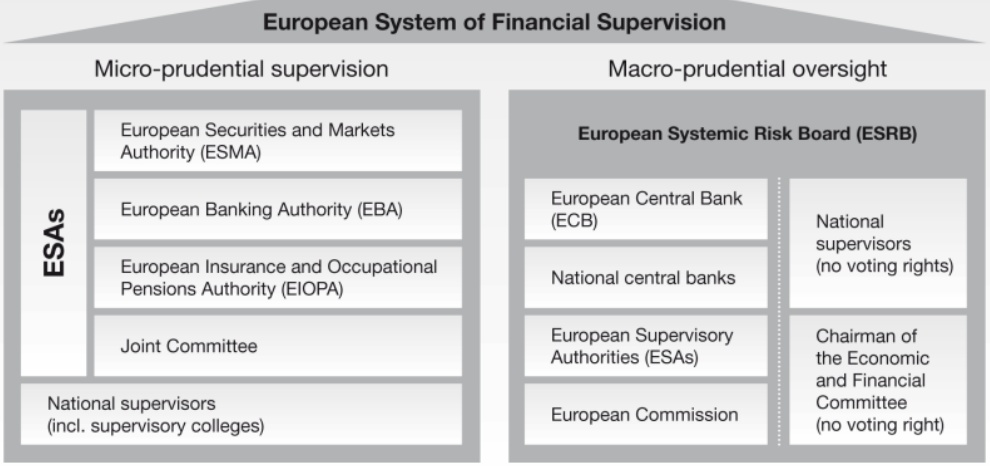

\section{Il Rischio Sistemico}

\subsection{Il Rischio Sistemico: possibili definizioni}

Il rischio sistemico non è univocamente definito a livello di letteratura e di regolamentazione. Esistono, infatti, diverse possibili accezioni del rischio sistemico, tutte ugualmente valide e condivisibili, che rendono la misurazione e la mitigazione di questo tipo di rischio complessa anche da un punto di vista teorico tanto che si potrebbe dire che per il rischio sistemico, più che per altre tipologie di rischio, vale il brocardo secondo cui è difficile definirlo, ma quando si verifica tutti concordano che si è verificato.

Le diverse possibili definizioni fanno riferimento a due macro-accezioni:

- un'accezione "ampia" che definisce il rischio sistemico secondo logiche potenzialmente applicabili anche a contesti estranei ai sistemi economici e finanziari;

- un'accezione "ristretta", che circoscrive il rischio sistemico a fenomeni di contagio interni al sistema bancario e finanziario, che si materializzano nel ben noto "effetto domino" secondo il quale il fallimento di un'istituzione finanziaria che abbia una sufficiente dimensione e livello di interconnessione col sistema può condurre al fallimento a catena di altre istituzioni.

Alla prima accezione possono appartenere manifestazioni del rischio sistemico che nulla hanno a che vedere col sistema finanziario, ad esempio la Grande Peste del Medioevo ${ }^{3}$ o altri eventi di tipo catastrofali per le conseguenze su larga scala che possono comportare.

Il caso Lehman ha indiscutibilmente fatto emergere la tematica del rischio sistemico con specifico riferimento al settore bancario e finanziario, attirando anche l'attenzione dei regolatori, rendendo evidente come il fallimento di un'istituzione grande e altamente interconnessa possa generare una crisi di tipo globale, provocando fallimenti a catena, evidenziando una delle modalità attraverso le quali il rischio sistemico può manifestarsi, che dà origine all'accezione "ristretta" di rischio sistemico.

Anche a causa di questi eventi, la definizione di rischio sistemico si è quindi indirizzata verso l'accezione "ristretta", al centro della quale c'è il concetto di contagio. Tuttavia, sebbene il rischio sistemico sia stato da quel momento oggetto di specifiche misure regolamentari è mancata e manca una definizione comunemente accettata.

Il Comitato di Basilea, nella sua revisione dell'Accordo del 2010 (la c.d. "Basilea 3"), ha indirizzato in maniera specifica i temi del rischio sistemico attraverso una serie di misure (in primis, ma non esclusivamente, i c.d. "buffer di capitale") ma non ne fornisce una definizione puntuale. Nel testo dell'Accordo si fa riferimento al rischio sistemico come:

- "derivante dall'interconnessione fra banche e altre istituzioni finanziarie tramite i mercati dei derivati"4;

- "presente nel settore finanziario, il Comitato innalza i fattori di ponderazione applicati alle esposizioni verso le istituzioni finanziarie rispetto a quelle verso imprese non finanziarie, essendo le prime maggiormente correlate rispetto a quelle non finanziarie con il fattore di rischio sistematico utilizzato per la stima dei requisiti dello schema di Basilea 2",

- connesso con il livello di interconnessione del sistema bancario e finanziario; ${ }^{6}$

- $\quad$ segnalato da ${ }^{7}$ un eccessivo incremento del credito erogato;

ma non ne fornisce una definizione. Nell'approccio del Comitato di Basilea, tuttavia, è evidente come il rischio sistemico sia direttamente legato all" "effetto domino" che si genera tramite un meccanismo di contagio che viene favorito dal grado di

${ }^{3}$ De Bandt, Hartman, European Central Bank, WORKING PAPER NO. 35 SYSTEMIC RISK: A SURVEY, 2000

${ }^{4}$ Comitato di Basilea per la vigilanza bancaria Basilea 3 - Schema di regolamentazione internazionale per il rafforzamento delle banche e dei sistemi bancari, Dicembre 2010 (d'ora in poi "Basilea 3"), paragrafo 14, comma c)

${ }^{5}$ Ibidem, paragrafo 14 , comma d)

${ }^{6}$ Ibidem, paragrafo 32

${ }^{7}$ Ibidem, paragrafo 138 
interconnessione del sistema bancario e finanziario. In altri termini, viene utilizzata una definizione "stretta" del rischio sistemico, che è anche alla base delle determinazioni del G-20 di Pittsburgh e delle iniziative del Financial Stability Board.

Altre fonti regolamentari adottano una definizione più ampia di quella del Comitato di Basilea, pur limitando il rischio sistemico al settore finanziario. Ad esempio, il Regolamento (UE) n 1092/2010 del Parlamento europeo e del Consiglio del 24 novembre 2010 definisce il rischio sistemico come: "un rischio di perturbazione del sistema finanziario che può avere gravi conseguenze negative per il mercato interno e l'economia reale. Tutti i tipi di intermediari, mercati e infrastrutture finanziari sono potenzialmente importanti in certa misura per il sistema".

Molto simile è la definizione contenuta nella Direttiva 2013/36/UE del Parlamento Europeo e del Consiglio del 26 giugno 2013 (d'ora in poi "CRD IV") che definisce il rischio sistemico come "un rischio di disordine del sistema finanziario che può avere gravi conseguenze negative per il sistema finanziario e l'economia reale".

\subsection{Rischio sistemico: una definizione operativa}

De Bandt e Hartman ${ }^{9}$ distinguono tra:

- evento sistemico in forma forte;

- evento sistemico in forma debole.

La differenza risiede negli effetti che l'evento sistemico, una volta verificatosi, è in grado di provocare.

Si parla di evento sistemico in forma forte se gli effetti dello stesso comportano il fallimento di istituzioni o il crash dei mercati che erano rispettivamente solventi e sani prima dell'evento stesso, e per i quali il fallimento o il crash non si sarebbero verificati senza l'evento stesso.

Si parla di evento sistemico in forma debole se l'effetto è di minore intensità rispetto ad un fallimento delle istituzioni o a un crash dei mercati.

Il rischio sistemico viene quindi definito come il rischio che accadano eventi sistemici in forma forte. Una crisi sistemica è quella generata dall'accadimento di un evento sistemico in forma forte che impatta un numero elevato di istituzioni e mercati, minando significativamente la funzionalità del sistema finanziario.

In base a queste definizioni, il fallimento di Lehman può essere considerato un evento sistemico in forma forte che ha generato una crisi sistemica. Non tutti gli eventi sistemici in forma forte generano necessariamente una crisi sistemica. Infatti, un evento sistemico in forma forte può provocare il fallimento di una o più istituzioni ma non necessariamente estendersi a un vasto numero di istituzioni o mercati.

Si parla, in questo caso di rischio di contagio, che può generare una crisi sistemica solo in presenza di determinate condizioni di interconnessione del sistema. Una similitudine efficace è quella di un'epidemia: questa può trasformarsi in una pandemia o estinguersi naturalmente dopo aver contagiato un numero limitato di individui in ragione della densità della popolazione del livello di contatto/interscambio tra individui (che corrisponde al concetto di interconnessione in ambito bancario/finanziario).

Per rendere la tematica trattabile da un punto di vista pratico oltre che concettuale, è necessario adottare una definizione di rischio sistemico coerente col contesto regolamentare e con gli obiettivi di questo position paper.

Pertanto, definiamo come rischio sistemico il rischio che il verificarsi di un evento sistemico in forma forte determini una crisi sistemica, come precedentemente definita.

Contestualizzata in ambito bancario/finanziario, questo significa che per rischio sistemico si intende il rischio che il fallimento di una o più istituzioni generi il fallimento a catena di un grande numero di altri istituzioni e/o il malfunzionamento dei mercati finanziari, tramite un meccanismo di contagio che potremmo definire di tipo "pandemico".

Questa definizione presenta l'indubbio vantaggio di essere coerente con l'approccio utilizzato dai regolatori che pur non definendo esplicitamente il rischio sistemico, ne dettano criteri di mitigazione che sono coerenti con la definizione adottata in questa sede.

\section{Gli strumenti di mitigazione del Rischio Sistemico}

Il rischio sistemico è per sua natura un rischio che può essere mitigato con misure di natura macro-prudenziale, che hanno come obiettivo la salvaguardia del sistema finanziario nel suo insieme e non con specifico riferimento ad un singolo istituto.

Lo European Systemic Risk Board (ESRB) ha identificato quattro potenziali fonti di rischio sistemico ${ }^{10}$ :

- eccessiva crescita del credito ed eccessiva leva finanziaria (A);

- eccessivo mismatch di scadenza e illiquidità dei mercati (B);

- eccessiva concentrazione (diretta o indiretta) (C);

- sistema di incentivi che favoriscono un "moral hazard" (con particolare riferimento alle istituzioni definite "a rilevanza sistemica") (D).

E' bene sin da subito evidenziare come la mitigazione di queste potenziali fonti di rischio sistemico abbia già comportato l'adozione di misure prudenziali nell'ambito di Basilea 3, siano esse già implementate o in corso di implementazione.

Rimandando alla seconda parte del presente position paper un'analisi di dettaglio delle misure intraprese nell'attuale framework, sia già implementate che in corso di implementazione, riportiamo sinteticamente quanto pubblicato dall'ESRB relativamente alle misure di mitigazione del rischio sistemico.

${ }^{8}$ CRD IV, Art. 3, comma 10

${ }^{9}$ De Bandt, Hartman, European Central Bank, WORKING PAPER NO. 35 SYSTEMIC RISK: A SURVEY, 2000

${ }^{10}$ The ESRB Handbook on Operationalising Macro-prudential Policy in the Banking Sector, pag. 7. 


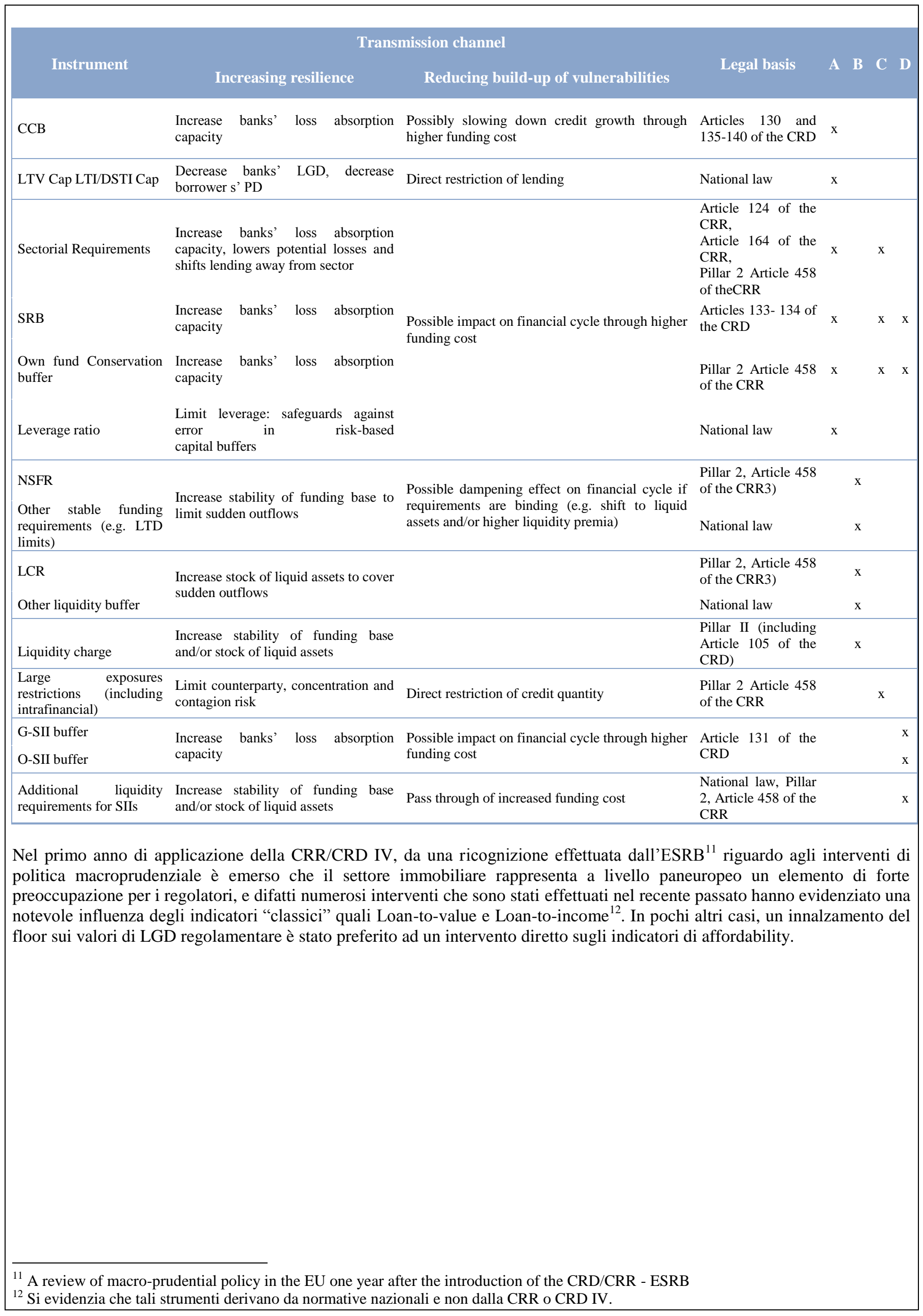




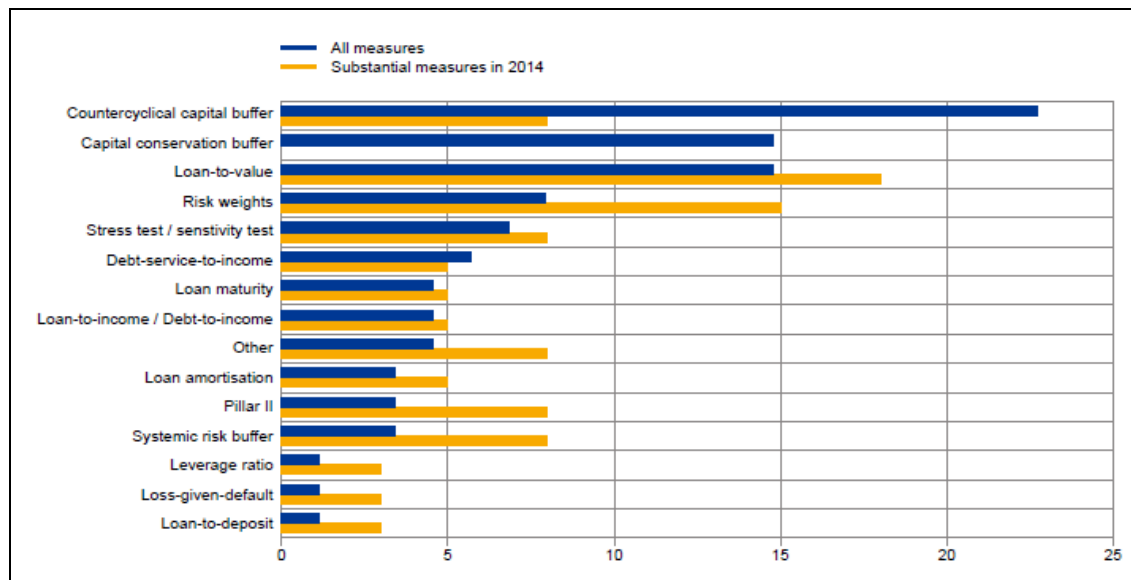

I policy tools come detto sono molteplici, e possono essere modulati per ottenere gli effetti desiderati sul livello complessivo di rischio sistemico.

\subsection{I fondi propri}

I Fondi Propri sono il principale strumento a disposizione della Banca per far fronte alle perdite sia in situazioni di "going concern" sia in situazioni di "gone concern"; rappresentano un presidio a fronte dei rischi legati alla normale attività bancaria e sono finalizzati a garantire la "viability" dell'Ente anche in condizioni avverse; rappresentano una garanzia che gli Enti offrono al mercato e, più in generale, al sistema economico al fine di tutelare dall'evento di default dell'Ente stesso e dal conseguente contagio.

Il Regulator ha posto molta enfasi ai fondi propri nel passaggio dalle regole di Basilea II a quelle di Basilea III, al fine di accrescere sia la quantità che la qualità stessa del capitale detenuto per fronteggiare i rischi tipici dell'attività bancaria. I fondi propri sono, pertanto, il primo strumento a copertura dei rischi bancari e conseguentemente a mitigazione del rischio sistemico, poiché contribuiscono sia alla riduzione della probabilità di default dell'Ente stesso sia a limitare la possibilità che il fallimento di un Ente si propaghi all'intero sistema economico e finanziario.

La quantità e la qualità dei Fondi Propri rappresentano, pertanto, uno dei fattori cardine della legislazione Europea e della Vigilanza Prudenziale. La quantità del Capitale detenuto dalle Banche dovrebbe essere sufficiente da ridurre al minimo la probabilità di insolvenza dell'Ente, mentre la sua qualità dovrebbe garantire la minimizzazione dei costi derivanti dagli impatti della crisi bancaria.

Il legislatore ha individuato due macro tipologie di strumenti di Capitale:

- Tier 1 finalizzati a garantire la continuità aziendale al di fuori di una situazione di crisi, ovvero in condizioni in cui la Banca può operare senza interventi esterni (Loss-absorbency on a going concern). Il Tier 1 è ulteriormente suddiviso in Common Equity Tier 1 ed Additional Tier 1;

- Tier 2 finalizzati a fronteggiare le perdite in situazione di crisi nelle quali è necessario ricorrere ad interventi esterni di gestione dello stato di crisi (Loss-absorbency on a gone concern).

L'ammissibilità degli strumenti finanziari tra i Fondi Propri dipende da quattro fattori: subordinazione, permanenza, flessibilità della remunerazione, capacità di assorbimento delle perdite. Le caratteristiche proprie del singolo strumento di capitale rispetto ai quattro fattori sopra riportati consentono la sua classificazione tra gli strumenti di Tier 1 e gli strumenti Tier 2.
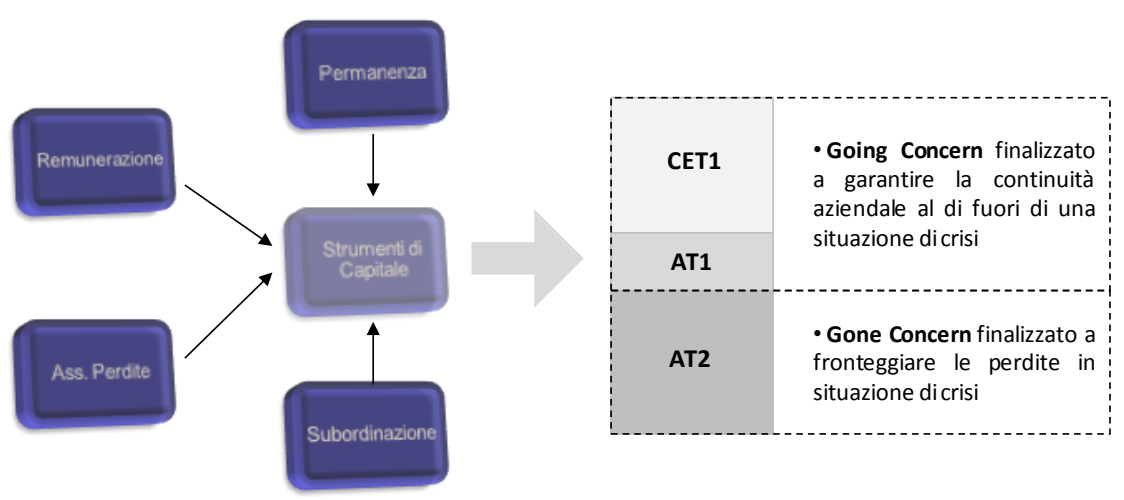

Le autorità competenti hanno il potere di richiedere agli enti di detenere un ammontare di Fondi Propri superiori ai requisiti minimi (art. 104 Direttiva 2013/36). La valutazione da parte delle Autorità di Vigilanza è effettuata a partire dei rilievi derivanti dal processo di verifica condotto dalle Autorità di Vigilanza (SREP). Ulteriori strumenti come il Macro stress testing potranno determinare requisiti superiori. 


\subsection{Capital buffers}

L'accordo di Basilea III introduce ulteriori capital buffers, ovvero cuscinetti aggiuntivi di capitale di elevata qualità (CET1) che la banca dovrà detenere, sopra ai minimi regolamentari, con molteplici finalità. La Direttiva 2013/36 introduce cinque capital buffers sintetizzati nella seguente tabella:

\begin{tabular}{|l|l|l|l|l|}
\hline \multicolumn{2}{|c|}{ CRD IV } & \multicolumn{2}{c|}{ Obiettivo finale } \\
Capital conservation buffer (CCB) & Art.129 & $\begin{array}{l}\text { Preservare i requisiti nei periodi } \\
\text { di stress }\end{array}$ & $\begin{array}{c}\text { Individuale e } \\
\text { consolidata }\end{array}$ & Note \\
\hline Countercyclical buffer (CCyB) & Art.130 & $\begin{array}{l}\text { Fronteggiare la componente } \\
\text { ciclica di rischio sistemico (pro } \\
\text { ciclicità requisisti capitale) }\end{array}$ & $\begin{array}{c}\text { Individuale e } \\
\text { consolidata }\end{array}$ & \\
\hline $\begin{array}{l}\text { Global systemically important } \\
\text { institution buffer (G-SII) }\end{array}$ & Art.131 & Ridurre l'effetto contagio e \\
quindi il rischio di “fallimenti a \\
catena"
\end{tabular}

La combinazione dei diversi buffer aggiuntivi determina, così come da Articolo 128 comma 6, il cosiddetto requisito combinato di riserva di capitale.

Nel caso la banca non rispetti i sopra menzionati vincoli potrà incorrere in misure sanzionatorie di vigilanza, come esplicitate dall'Articolo 141, quali il divieto alla distribuzione degli utili o al pagamento di bonus di gestione al personale. L'articolo 141 si applica al Capital Conservation Buffer, al Countercyclical Buffer e al Systemic Risk buffer, nonché al requisito combinato di riserva di capitale.

\subsection{Requisiti finanziari}

La recente crisi finanziaria ha reso evidenti non solo rilevanti fattori di fragilità sistemica insiti nella struttura, nella composizione e nei modi di operare dei sistemi finanziari (mercati e intermediari), ma anche alcune importanti criticità dell'impianto regolamentare di Basilea II. Il rischio di liquidità, prima della crisi finanziaria, non è mai stato collocato al centro degli standard vincolanti internazionali, sulla base della convinzione che le esigenze degli intermediari finanziari potessero essere fronteggiate utilizzando i mercati interbancari. La protratta fase di recessione ha richiamato l'attenzione delle autorità di vigilanza verso la necessità di un'adeguata gestione del rischio di liquidità per garantire la stabilità degli intermediari e del sistema nel suo complesso.

Il Comitato di Basilea ha inteso correggere alcuni punti della regolamentazione prudenziale messi in evidenza dalla crisi, successivamente recepiti all'interno della Regolamentazione Europea (parte VI del Regolamento UE 575/2013).

Per dare una decisiva impronta di rivoluzionamento ai sistemi di liquidity risk management e rafforzare la capacità del settore bancario di resistere a crisi di liquidità il Comitato di Basilea 3 ha elaborato due requisiti minimi obbligatori di liquidità:

- $\quad$ Liquidity Coverage Ratio (LCR);

- Ne Stable funding Ratio (NSFR);

Il primo indicatore di liquidità impone alla banca di avere sufficienti "attivi liquidi di alta qualità" (HQLA) non vincolati, composti da contanti e attività che possano essere convertiti in contante con una perdita modesta o nulla, per soddisfare il fabbisogno di liquidità nell'arco di 30 giorni in uno scenario di stress predefinito.

$$
L C R=\frac{\text { High Quality Liquid Assets }}{\text { Net Cash Outflows over a } 30-\text { day period }} \geq 100 \%
$$

Il secondo indicatore di liquidità, il NSFR, mira a garantire una struttura equilibrata tra poste attive e passive di bilancio nell'orizzonte temporale di un anno. Il NSFR vuole scongiurare il cosiddetto "effetto precipizio" dopo il mese, che si potrebbe manifestare considerando unicamente la regola di breve termine.

$$
\text { NSFR }=\frac{\text { Available Stable Funding }}{\text { Required Stable Funding }} \geq 100 \%
$$




\subsection{Add-on di capitale e di liquidità}

L'Autorità di Vigilanza può richiedere agli Enti di detenere requisiti di Capitale e di Liquidità superiori a quelli minimi previsti (art. 104 e 105 della Direttiva 2013/36) al fine di coprire tutti i rischi ai quali un Ente sia o possa essere esposto, tenendo conto dei seguenti elementi:

- Per quanto attiene il Capitale:

$\checkmark$ gli aspetti quantitativi e qualitativi del processo di valutazione di un ente (capitale interno);

$\checkmark \quad$ i dispositivi, i processi e i meccanismi di un ente (Governance interna e piani di risanamento e risoluzione);

$\checkmark$ il risultato della revisione e della valutazione effettuate (SREP);

$\checkmark$ la valutazione del rischio sistemico.

- Per quanto attiene la Liquidità:

$\checkmark$ il modello imprenditoriale dell'ente;

$\checkmark$ i dispositivi, i processi e i meccanismi dell'ente (rischio di liquidità);

$\checkmark$ il risultato della revisione e della valutazione (SREP);

$\checkmark$ il rischio di liquidità sistemico che minaccia l'integrità dei mercati finanziari dello Stato membro interessato.

Gli add-on di capitale e di liquidità sono pertanto determinati in funzione sia degli esiti dello SREP che di ulteriori valutazioni effettuate dal Supervisors in merito al rischio sistemico dell'Ente.

Per quanto attiene quest'ultimo aspetto, non esistono metodologie univoche e ben specifica teda parte dei Supervisors. A giudizio della Commissione è tuttavia possibile annoverare almeno i seguenti strumenti: il CISS e il Macro Stress Testing.

\subsubsection{ICAAP, ILAAP e SREP}

In merito allo SREP, come noto l'EBA ha pubblicato, conformemente all'articolo 107 della CRD, una Guidelines ${ }^{13}$ con il compito di uniformare le revisioni e le valutazioni prudenziali e le misure di vigilanza.

Lo SREP si basa su quattro macro aree:

- Valutazione del business model;

- Governance e sistemi di controllo;

- ICAAP;

- ILAAP;

Per ciascun ambito è espresso un giudizio da 1-4 o un giudizio negativo $(\mathrm{F})$ nel caso in cui si ritenga che la banca sia classificabile tra quelle "Failing or likely to fail". Nel caso di giudizio pari a F si attiva l'articolo 32 della BRRD (condizioni di risoluzione)

Una corretta implementazione dello SREP risulta, quindi, fondamentale come strumento di mitigazione del rischio sistemico, andando, in ultima istanza, a fornire, attraverso un sistema di scoring, una valutazione sull'overall risk dell'istituto e prescrivendo, interventi correttivi ove necessario. In particolare, per quanto riguarda l'assessment dell'adeguatezza patrimoniale lo SREP procede a fornire una quantificazione dei cosiddetti Total Srep Capital Requirements. In tal senso è auspicabile un'attenta valutazione da parte del Regulator ai fini di evitare fenomeni di double counting tra le varie misure del suddetto processo di Pillar II, ossia add-on di fondi propri, della CRD IV e di altre prescrizioni macro-prudenziali.

In quest'ottica ben si comprende come lo SREP costituisca un ulteriore strumento nelle mani del Regulator per procedere verso quell'auspicata armonizzazione di misure micro e macro-prudenziali di mitigazione del rischio sistemico.

\subsubsection{CISS}

A livello complessivo uno strumento utilizzabile dal Regulators può essere rappresentato dal CISS, ovvero un indicatore sintetico di rischio sistemico creato dalla BCE che analizza l'andamento complessivo del sistema finanziario ${ }^{14}$. Nei grafici successivi sono riportati l'andamento del CISS a livello di Unione Europea (grafico di sinistra) e è per singoli paesi UE (grafico di destra):
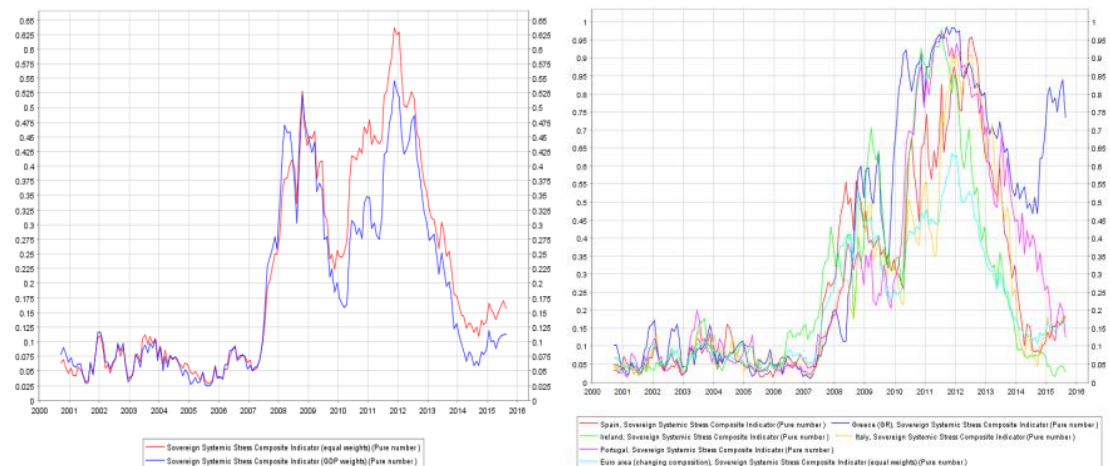

${ }^{13}$ Guidelines on common procedures and methodologies for the supervisory review and evaluation process (SREP). EBA 19 Dicembre 2014.

${ }^{14}$ CISS - A Composite Indicator of Systemic Stress in the Financial System - BCE 
E' verosimile attendersi un comportamento dei Regulators maggiormente aggressivo nei confronti di quegli istituti appartenenti ad uno Stato il cui rischio sistemico risulti in aumento o comunque al di sopra della media dell'Unione Europea.

\subsubsection{Il Macro Stress Testing}

A livello di singolo istituto, il principale strumento di analisi del rischio sistemico è il Macro Stress Testing condotto dalle Autorità di Vigilanza, finalizzato a valutare la resilienza del singolo istituto ad eventi macro economici avversi particolarmente severi in ottica forward looking: "Macro stress tests are a tool to measure the resilience of the financial system or its key components to various stress factors, based on the quantification of the link between macroeconomic variables and the health of either individual financial institutions or the financial sector as a whole",15.

Gli esercizi di stress testing sono oramai annoverati tra i tools che le Autorità di Vigilanza hanno per valutare le condizioni del sistema finanziario e la sua tenuta a fronte di eventi di stress.

Il FMI utilizza regolarmente, da anni, gli stress testing nell'ambito del proprio Financial Sector Assessment Programs (FSAPs). Le autorità di Vigilanza Europee e Americane hanno negli anni scorsi avviato diversi stress testing sui principali istituti bancari. Come noto, il recente EU wide Stress Testing condotto dalla BCE nell'ambito del Comprehensive assessment ha comportato richieste specifiche di add-on di capitale (capital decision) da parte delle Autorità di Vigilanza.

\subsection{I piani di Recovery e Resolution plan}

La direttiva BRRD (direttiva 2014/59 UE c.d. Bank Recovery and Resolution Directive) introduce in tutti i paesi europei regole armonizzate per prevenire e gestire le crisi delle banche e delle imprese di investimento in modo ordinato attraverso strumenti efficaci e risorse provenienti dal settore privato al fine di spezzare il legame esistente tra crisi bancarie e crisi del debito sovrano. La BRRD completa, come detto, l'Unione Bancaria con il Meccanismo unico di risoluzione (SRM).

L'SRM è un sistema articolato che si compone delle autorità di risoluzione nazionali e di un'autorità accentrata, il Comitato Unico di Risoluzione (Single Resolution Board, SRB), cui partecipano rappresentanti delle autorità di risoluzione nazionali e alcuni membri permanenti e a cui spetta il compito di individuare le modalità di gestione della crisi per le banche soggette all'SSM.

La procedura è gestita dalle Autorità di risoluzione delle crisi ai quali sono affidati i seguenti compiti:

- pianificare la gestione delle crisi prevedendo misure di prevenzione della medesima, che devono essere predisposte nella fase di normale svolgimento dell'attività delle imprese finanziarie quali:

$\checkmark$ i piani di risanamento (recovery plan) redatti a cura degli Enti stessi, ma validati dall' autorità di supervisione;

$\checkmark \quad$ i piani di risoluzione (resolution plan), preparati dalle Autorità preposte alla risoluzione delle crisi;

- intervenire per tempo, prima della completa manifestazione della crisi, per assicurare azioni tempestive da parte delle autorità di risoluzione, una volta che si siano manifestati i primi segnali di rischio per la stabilità degli enti creditizi e delle imprese di investimento;

- gestire la fase di "risoluzione". Tra gli strumenti annoverati rientrano:

$\checkmark$ cessione di una parte dell'attività a un acquirente privato;

$\checkmark$ trasferire temporaneamente le attività e passività a un'entità (bridge bank) costituita e gestita dalle autorità per proseguire le funzioni più importanti, in vista di una successiva vendita sul mercato;

$\checkmark$ trasferire le attività deteriorate a un veicolo (bad bank) che ne gestisca la liquidazione in tempi ragionevoli;

$\checkmark$ applicare il bail-in, ossia svalutare azioni e crediti e convertirli in azioni per assorbire le perdite e ricapitalizzare la banca in difficoltà o una nuova entità che ne continui le funzioni essenziali;

$\checkmark$ le perdite non assorbite dai creditori esclusi in via discrezionale possono essere trasferite al fondo di risoluzione (alimentato negli anni da contributi versati dalle banche dei paesi partecipanti e progressivamente mutualizzati) che può intervenire nella misura massima del 5 per cento del totale del passivo, a condizione che sia stato applicato un bail-in minimo pari all's per cento delle passività totali.

$\checkmark$ intervento pubblico (anche mediante nazionalizzazione temporanea dell'Istituto) da attivarsi solo dopo che il bail-in degli investitori privati abbia raggiunto l' $8 \%$ del totale del passivo.

Come noto il bail-in consente alle autorità di risoluzione di disporre della riduzione del valore delle azioni e di alcuni crediti o la loro conversione in azioni per assorbire le perdite e ricapitalizzare la banca in misura sufficiente a ripristinare un'adeguata capitalizzazione e a mantenere la fiducia del mercato.

Il bail-in si applica ad azionisti e creditori in funzione di uno specifico ordine di priorità:

1. azionisti;

2. detentori di altri titoli di capitale;

3. altri creditori subordinati;

4. creditori chirografari;

5. persone fisiche e le piccole e medie imprese titolari di depositi per l'importo eccedente i 100.000 euro;

6. fondo di garanzia dei depositi, che contribuisce al bail-in al posto dei depositanti protetti (cioè fino a 100.000 euro).

${ }^{15}$ Financial Stability Review, December 2010, Special Feature A for a discussion of the use of stress tests during a crisis - BCE 
E' prevista la possibilità da parte dell'Autorità di escludere, previa approvazione da parte della Commissione Europea, alcune passività qualora l'applicazione dello strumento comporti un rischio per la stabilità finanziaria o comprometta la continuità di funzioni essenziali.

\subsection{TLAC E MREL}

Nel framework normativo europeo sempre più caratterizzato dall'attenzione verso una maggiore armonizzazione degli interventi regolamentari micro e macro-prudenziali, vanno certamente ad occupare un posto di rilievo la Direttiva 2014/59/UE, emanata dall'EBA e contente il cosiddetto Minimum Requirement for Eligible Liabilities, e lo strumento del Total Loss Absorbing Capacity, predisposto quest'ultimo dal Financial Stability Board.

Analizzando i due strumenti proposti, rispettivamente, dall'EBA e dal FSB emergono alcune differenze, che attraverso questo paragrafo si è tentato di riassumere. Innanzitutto, se MREL si rivolge a tutti gli istituti e banche d'investimento, il requisito di TLAC sarà vincolante solo, almeno per il momento, per le c.d. G-SIBs. Ad un approccio case by case per il calcolo del MREL, basato su considerazioni relative, ad esempio, alla resolvability, al risk assessment ed al risk profile, si contrappone l'applicazione a tutti gli istituti di credito della medesima soglia di TLAC, per quanto concerne la parte di requisito relativa al Pillar I, mentre un approccio firm-specific viene seguito per la prescrizione della parte di TLAC relativa al requisito di Pillar II. Per MREL ci si attende, perciò, una calibrazione ad-hoc da parte delle Autorità di Vigilanza nazionali, che vada a considerare i fattori idiosincratici della singola banca. Inoltre, mentre il requisito MREL viene espresso sotto forma di percentuale di propri fondi e passività totali, TLAC è costituito da un requisito di capitale o dal leverage ratio, in particolare il 16-20\% delle RWA più la parte di Pillar II. Infine differenti risultano i processi che avranno luogo nel caso di superamento delle soglie imposte dai medesimi. Uno sforamento dal requisito MREL non implica l'attivazione di automatismi, mentre, dall'altro lato, il mancato rispetto di TLAC è considerato alla stregua di un infrangimento dei requisiti minimi di capitale e, come tale, può attivare un processo di risoluzione.

In particolare, ci si vuole soffermare sulla costruzione dell'indicatore TLAC. Nell'ordine le varie parti che lo compongono sono:

- CET1;

- Additional Tier 1;

- Tier2;

- Long Unsecured Debt;

Quest'ultima parte, in particolare, può esser ridotta grazie a Tier2 o Additional Tier 1 in eccesso, sempre garantendo che l'ammontare di Long Unsecured Debt rimanga almeno il 33\% del TLAC. Come si evince, il Buffer di Conservazione di Capitale viene a formarsi oltre al requisito di TLAC in quanto solo CET1 in eccesso oltre al TLAC può andare a costituire $\mathrm{i}$ buffer di capitale. In altre parole, se il debito in scadenza conteggiato nel TLAC non viene rinnovato, il primo ad essere infranto sarà il rispetto del buffer di capitale, con tutte le conseguenze che ne derivano, dal momento che rispettare il buffer di capitale è condizione necessaria per poter distribuire i dividendi agli azionisti, bonus ai dipendenti e coupon ai possessori di debito AT1.

In ultima istanza MREL e TLAC si differenziano per data di implementazione, dovendo il primo entrare in vigore nel 2016, mentre il secondo nel 2019.

\subsection{Altri strumenti di mitigazione del rischio sistemico}

In questa categoria rientrano un ampia ed articolata serie di strumenti utilizzabili dai Regulators quali:

- Requisiti patrimoniali specifici per settore;

- Limiti obbligatori al loan to value (LTV);

- Limiti obbligatori al rapporto prestiti/redditi o (servizio del) debito/reddito (loan to income, LTI);

- Restrizioni alle grandi esposizioni;

Per quanto attiene i requisiti patrimoniali specifici per settore l'approccio previsto dal Regolatore prevede la modifica del calcolo dei requisiti per specifici settori o categorie di attività lasciando tale facoltà alla Competent Authority che potrà fissare requisiti più elevati qualora si registri un rischio per il sistema finanziario nel suo complesso. A titolo esemplificativo, per le esposizioni garantite da ipoteche su immobili residenziali, l'autorità competente potrà fissare il fattore di ponderazione del rischio in una percentuale variabile tra il $35 \%$ ed il $150 \%$ o tra il $50 \%$ ed il $150 \%$ per le esposizioni garantite da immobili non residenziali. Ulteriori requisiti più stringenti potranno essere fissati anche con riferimento all'LGD (soglie minime superiori al $10 \%$ e al $15 \%)$.

I limiti obbligatori per il LTV e per il LTI possono essere introdotti al fine di moderare il ciclo creditizio e accrescere la capacità di tenuta degli istituti finanziari. Seppure applicati in vari paesi dell'UE, i limiti al LTV ed al LTI non sono armonizzati a livello di Unione Europea.

Come noto le grandi esposizioni sono quelle che hanno un valore pari o superiore al $10 \%$ dei fondi propri dell'ente. La normativa di Basilea III prevede anche che gli Stati membri possano definire soglie specifiche verso settori a rilevanza sistemica in considerazione della loro rischiosità. 


\section{Quantificazione dei requisiti patrimoniali}

Le modifiche introdotte da Basilea III hanno sostanzialmente incrementato l'ammontare dei fondi propri da detenere a fronte dei rischi assunti, sia mediante l'introduzione di buffers sia mediante richieste di ulteriori additional own funds da parte dei Regulators in funzione dell'esito delle verifiche ispettive (SREP) o degli esercizi di stress test. Basilea III non ha, tuttavia, modificato in maniera sostanziale i criteri di calcolo dei requisiti patrimoniali, che già in ottica Basilea II presentavano notevoli criticità per le banche che spesso erano chiamate a sovrastimare il rischio e quindi anche le dotazioni patrimoniali. Emblematiche sono le modalità di calcolo del requisito patrimoniale a fronte del rischio di mercato del trading book o quelle del requisito patrimoniale a fronte del rischio di credito che si basano su stime di correlazione mai aggiornate dall'introduzione di Basilea II e relative a dati storici antecedenti il 2004.

Nel proseguo dell'analisi ci si focalizzerà prevalentemente sui requisiti patrimoniali sul rischio di credito (rappresentativi di circa il $90 \%$ del totale dei requiti patrimoniali), per il quale si è provveduto ad una ristima delle asset correlation mediante un approccio proposto da Fitch Rating ${ }^{16}$. Per gli altri requisiti patrimoniali si è utilizzato un approccio qualitativo in considerazione dello scarso contributo al requisito patrimoniale complessivo.

\subsection{Il rischio di credito}

Nell'ambito dei metodi avanzati, il calcolo degli RWA per il rischio di credito discerne da una serie di funzioni di ponderazione $^{17}$, sviluppate e calibrate a partire dalle metodologie di stima della perdita inattesa (c.d. modelli di Credit VaR alla Merton).

Il Modello di portafoglio regolamentare (ASRF - Asymptotic Single Risk Factor Model) si basa sull'assuzione di invarianza del portafoglio (Portfolio Invariant) che consente di determinare il VaR come somma additiva dei singoli VaR. Questa ipotesti implica che la perdita inattesa di una singola esposizione non dipenda dal contributo marginale della stessa al rischio complessivo del portafoglio. Esso è pertanto funzione dei soli parametri di PD, LGD, EAD e Maturity.

La scelta di assumere l'invarianza di portafoglio consente di:

1. trascurare le correlazioni tra i rendimenti delle attività delle singole controparti (si suppone che le controparti abbiamo la medesima correlazione pari alla radice quadrata del factor loading wi);

2. semplificare il calcolo dei requirement normativi

L'approccio asintotico monofattoriale richiede tuttavia due condizioni:

1. il portafoglio sia infinitamente granulare ovvero:

a. numero di controparti tendente ad infinito;

b. l'esposizione della i-esima controparte sul totale del portafoglio abbia un peso prossimo a zero (tale ipotesi deriva dalla precedente ed implica che in presenza di controparti illimitate, anche qualora vi fosse una disomogeneità nelle esposizioni di portafoglio, il peso percentuale di ogni esposizione sul totale di portafoglio tenda sempre a zero).

2. Il default della controparte dipende da un unico fattore (fattore sistematico) la cui sensitività (correlazione rispetto al fattore sistematico) è costante per tutte le controparti (pertanto le controparti risultano perfettamente correlate tra loro).

Nel modello ASRF, la perdita attesa per unità di esposizione del portafoglio, condizionata al verificarsi di una determinazione $\mathrm{X}=\mathrm{x}$ del fattore sistematico è pari $\mathrm{a}$ :

$$
E\left[L_{n} \mid X=x\right]=\frac{\sum_{i=1}^{n} E A D_{i} \cdot P D_{i}(x) \cdot L G D_{i}}{\sum_{i=1}^{n} E A D_{i}}
$$

dove:

Ln la variabile casuale perdita

$\mathrm{X}$ è il fattore sistematico (l'andamento dell'economia)

Come detto alla base del modello ASRF ci sono le seguenti ipotesi:

1. la perdita attesa è una funzione strettamente monotona in $\mathrm{x}$;

2. la distribuzione delle perdite per unità di esposizione coincide con il suo valore atteso condizionato $L_{n}-E\left[L_{n} \mid X=x\right] \rightarrow 0$

3. per $n \rightarrow \infty$, l'ammontare di ciascuna esposizione è trascurabile rispetto al totale delle esposizioni in portafoglio (infinità granularità del portafoglio).

Solo sotto queste ipotesi vale il seguente teorema limite per il $\mathrm{VaRq}^{18}$ del portafoglio per unità di esposizione:

${ }^{16}$ Basel II Correlation Values - An Empirical Analysis of EL, UL and the IRB Model. Fitch Rating

${ }^{17} \mathrm{Si}$ veda al riguardo Michel B. Gordy (2002) "A Risk-Factor Model Foundation for Ratings-Based Bank Capital Rules".

${ }^{18}$ Calcolato in corrispondenza di un livello di probabilità q e lungo un orizzonte temporale annuo. 


$$
\lim _{n \rightarrow \infty} \operatorname{VaR}_{q}\left[L_{n}\right]=E\left[L_{n} \mid X=x_{q}\right]=\frac{\sum_{i=1}^{n} E A D_{i} \cdot P D_{i}\left(x_{q}\right) \cdot L G D_{i}}{\sum_{i=1}^{n} E A D_{i}}
$$

Il VaR determinato mediante il modello ASRF è pertanto pari al prodotto tra l'LGD e la PD condizionata al verificarsi di un valore estremamente avverso del fattore sistematico X (pari al 99,9\%).

Il calcolo di tale probabilità di insolvenza si basa su un modello alla Merton:

$$
Y_{i}=w_{i} \cdot X+\sqrt{1-w_{i}^{2}} \cdot \varepsilon_{i}
$$

dove:

Yi è il rendimento delle attività del singolo debitore;

$\mathrm{X}$ è il fattore sistematico;

$\varepsilon_{i}$ è il fattore idiosincratico;

wi è il coefficiente di sensitività al rischio sistematico ("factor loading").

Quando il valore delle attività del debitore Yi scendono al disotto di una certa soglia Z scatta l'evento di default del debitore $(\mathrm{Yi}<\mathrm{Z})$. Analiticamente:

$$
P\left\{Y_{i}<Z \mid X=x_{q}\right\}=P\left\{w_{i} X+\sqrt{\left(1-w_{i}^{2}\right)} \varepsilon_{i}<Z \mid X=x_{q}\right\}=P\left\{\varepsilon_{i}<\frac{Z-w_{i} x_{q}}{\sqrt{1-w_{i}^{2}}}\right\}=N\left(\frac{Z-w_{i} x_{q}}{\sqrt{1-w_{i}^{2}}}\right)
$$

Il modello assume che il rendimento delle attività del debitore si distribuiscano come una normale $(\mathrm{N})$ e, pertanto, $Z=N^{-1}(P D)=G(P D)$

$$
L G D \cdot N\left(\frac{1}{\sqrt{1-w_{i}^{2}}} G(P D)+\frac{w_{i}}{\sqrt{1-w_{i}^{2}}} G(0.999)\right)=L G D \cdot N\left(\frac{1}{\sqrt{1-\rho}} G(P D)+\sqrt{\frac{\rho}{1-\rho}} G(0.999)\right)
$$

Un elemento centrale nella formula utilizzata dal Regolatore e, quindi, nel calcolo dei requisiti patrimoniali è il parametro di correlazione wi. Una elevata correlazione implica anche un maggior assorbimento di capitale. La correlazione fornita dal regolatore varia generalmente tra 1 ' $8 \%-24 \%$ per la curva di ponderazione c.d. Corporate, e nell'intervallo 3\%-16\% per quelle c.d. Retail.

La stima delle correlazioni proposta dal Comitato di Basilea già nel $2004^{19}$ non ha subito mai modifiche con esclusione degli interventi relativi allo Sme Supporting Factor o quelli sulle Asset Value Correlation.

Nel proseguo del presente paragrafo si vuole approfondire il rapporto tra le Pd e le asset correlation, basato sul sistema bancario italiano. L'obiettivo è, pertanto, verificare se le correlazioni fornite dal regolatore consentono una corretta quantificazione dei requisiti patrimoniali.

L'approccio utilizzato prevede la ri-stima delle correlazione regolamentari attraverso una misura di correlazione implicita che eguagli la perdita inattesa empiricamente osservata con i requisiti patrimoniali di vigilanza ${ }^{20}$.

La correlazione implicita è stata ricavata utilizzando una distribuzione Beta. La distribuzione Beta è una distribuzione di probabilità continua definita da due parametri $\alpha \mathrm{e} \beta$ su un intervallo unitario compreso tra 0 ed 1 . I due parametri possono essere interpretati come l'inclinazione della "gobba" (ovvero la concentrazione in termini di probabilità dei tassi di perdita attorno al valore medio) e dello "spessore" (ovvero la probabilità di ottenere tassi di perdita significativamente più elevati di quelli medi).

La distribuzione beta è descritta dalla seguente densità di probabilità:

$$
P(x)=\frac{\Gamma(\alpha+\beta)}{\Gamma(\alpha) \cdot \Gamma(\beta)} \cdot \int_{0}^{x}(1-t)^{\beta-1} \cdot t^{\alpha-1} d t
$$

con:

$$
\begin{aligned}
& \alpha=\mu \cdot\left(\frac{1-\mu}{\sigma^{2}}\right)-1 \\
& \beta=(1-\mu) \cdot\left(\frac{1-\mu}{\sigma^{2}}\right)-1
\end{aligned}
$$

dove:

$\mu=$ media della perdita attesa empirica;

$\sigma^{2}$ deviazione standard della perdita attesa empirica;

${ }^{19}$ International Convergence of Capital Measurement and Capital Standards - BIS, document poi rivisto e confluito nel testo finale dell'accordo di Basilea II del 2006 Basel II: International Convergence of Capital Measurement and Capital Standards: A Revised Framework

${ }^{20}$ Basel II Correlation Values - An Empirical Analysis of EL, UL and the IRB Model. Fitch Rating 
$\Gamma$ è la distribuzione Gamma.

Ipotizzando che la perdita attesa empirica si distribuisca secondo una distribuzione Beta è stato determinato il valore della UL (perdita inattesa) empirica.

L'asset correlation è stata derivata calcolando il valore di correlazione che eguaglia la UL (calcolata secondo le funzioni di ponderazione Basel III) con la UL empirica.

Le strutture di correlazione coinvolte appaiono generalmente più prudenziali di quanto ipotizzabile in prima battuta, con la volontà più o meno esplicita di catturare effetti difficilmente quantificabili legati proprio al rischio sistemico. Il regolatore si prefigge quindi lo scopo di mitigarne il rischio tramite una maggiore quantità del capitale da detenere supponendo correlazioni maggiori di quelle effettivamente registrate.

Per la serie storica della perdita attesa sono stati utilizzate le PD e le LGD disponibili nelle diverse informative al Pubblico.

Le analisi sono state effettuate considerando i seguenti portafogli regolamentari:

1. Esposizioni al dettaglio: Esposizioni garantite da immobili residenziali: PMI

2. Esposizioni al dettaglio: Esposizioni garantite da immobili residenziali: Persone Fisiche

3. Esposizioni al dettaglio: Esposizioni rotative al dettaglio qualificate

4. Esposizioni al dettaglio: Altre: PMI

5. Esposizioni al dettaglio: Altre: Persone Fisiche

6. Esposizioni verso o garantite da imprese - PMI

7. Esposizioni verso o garantite da imprese - Altre Imprese

Per ciascun portafoglio è stata calcolata la correlazione implicita, che successivamente è stata applicata alle diverse funzione di poderazione. La differenza tra l' Unexpected Loss implicita (con correlazione stimata a partire dalla distribuzione beta) e l' Unexpected Loss calcolata con la formula di Gordy consente di ricavare l'extra requisito patrimoniale (delta Unexpected Loss). Tale valore rappresenta un add-on di requisito patrimoniale per ogni euro di EAD.

Per ciascun portafoglio sono state effettuate delle analisi di sensitività (incremento di +50/+100 bps della PD, decremento della PD di -50/-100 bps, LGD fissa al 45\%).

Di seguito si riporta l'esito delle analisi e le relative CDF e PDF della distribuzione beta:

Esposizioni al dettaglio: Esposizioni garantite da immobili residenziali: PMI

\begin{tabular}{|c|c|c|c|c|}
\hline & Dati Effettivi & LGD $45 \%$ & PD - 100bps & $\mathrm{PD}+100 \mathrm{bps}$ \\
\hline PD & $5,60 \%$ & $5,60 \%$ & $4,60 \%$ & $6,60 \%$ \\
\hline LGD & $20,00 \%$ & $45,00 \%$ & $20,00 \%$ & $20,00 \%$ \\
\hline EL & $1,12 \%$ & $2,52 \%$ & $0,92 \%$ & $1,32 \%$ \\
\hline Corr Implicita & $10,93 \%$ & $2,40 \%$ & $13,87 \%$ & $8,91 \%$ \\
\hline Corr Basel & $15,00 \%$ & $15,00 \%$ & $15,00 \%$ & $15,00 \%$ \\
\hline UL Implicita & $4,62 \%$ & $3,56 \%$ & $5,01 \%$ & $4,33 \%$ \\
\hline UL Basel & $5,92 \%$ & $13,32 \%$ & $5,35 \%$ & $6,41 \%$ \\
\hline Delta UL & $1,30 \%$ & $9,76 \%$ & $0,33 \%$ & $2,08 \%$ \\
\hline
\end{tabular}

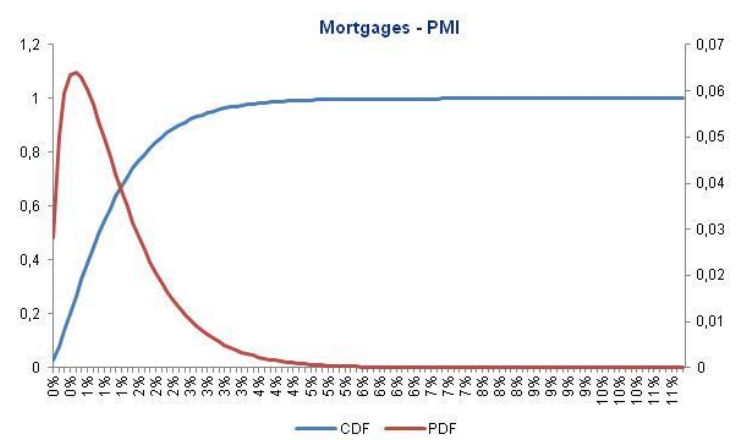




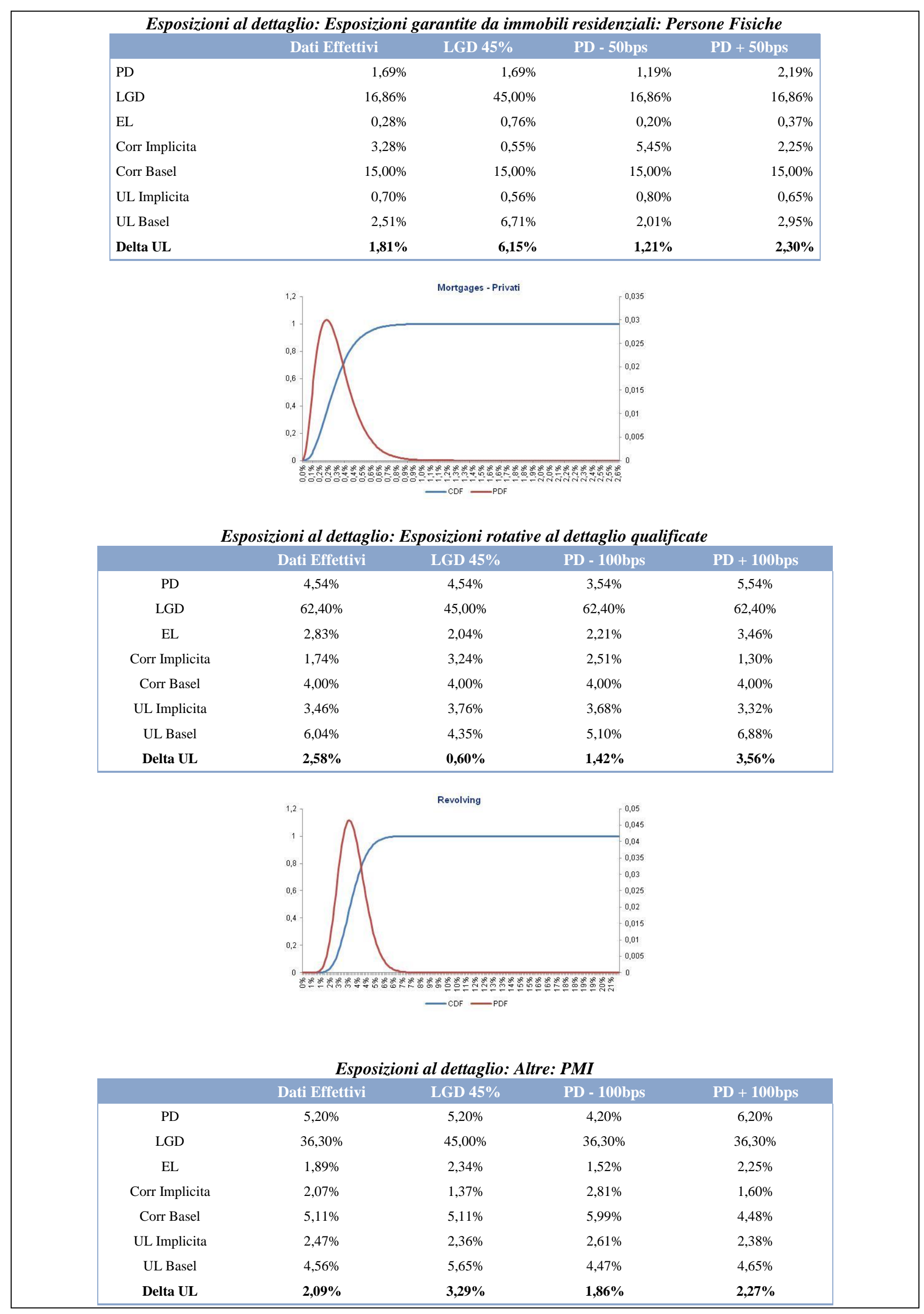




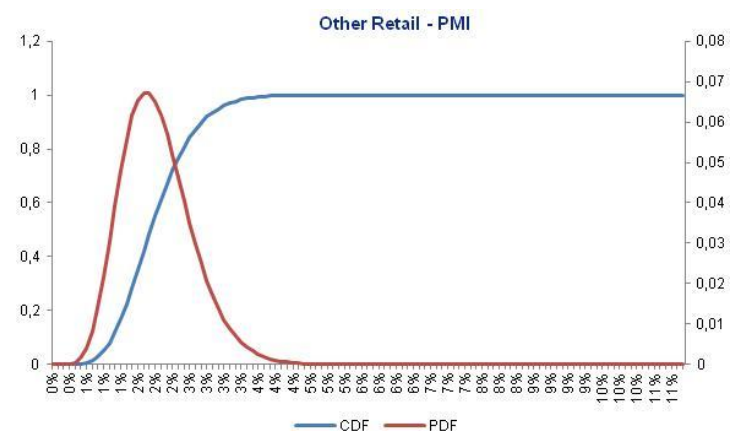

Esposizioni al dettaglio: Altre: Persone Fisiche

\begin{tabular}{|c|c|c|c|c|}
\hline & Dati Effettivi & $\mathrm{LGD} 45 \%$ & PD - 100bps & PD + 100bps \\
\hline $\mathrm{PD}$ & $3,05 \%$ & $3,05 \%$ & $2,05 \%$ & $4,05 \%$ \\
\hline LGD & $40,78 \%$ & $45,00 \%$ & $40,78 \%$ & $40,78 \%$ \\
\hline EL & $1,24 \%$ & $1,37 \%$ & $0,84 \%$ & $1,65 \%$ \\
\hline Corr Implicita & $0,85 \%$ & $0,71 \%$ & $1,57 \%$ & $0,55 \%$ \\
\hline Corr Basel & $7,47 \%$ & $7,47 \%$ & $9,34 \%$ & $6,15 \%$ \\
\hline UL Implicita & $1,07 \%$ & $1,06 \%$ & $1,17 \%$ & $1,03 \%$ \\
\hline UL Basel & $4,84 \%$ & $5,34 \%$ & $4,48 \%$ & $5,00 \%$ \\
\hline Delta UL & $3,76 \%$ & $4,28 \%$ & $3,32 \%$ & $3,98 \%$ \\
\hline
\end{tabular}

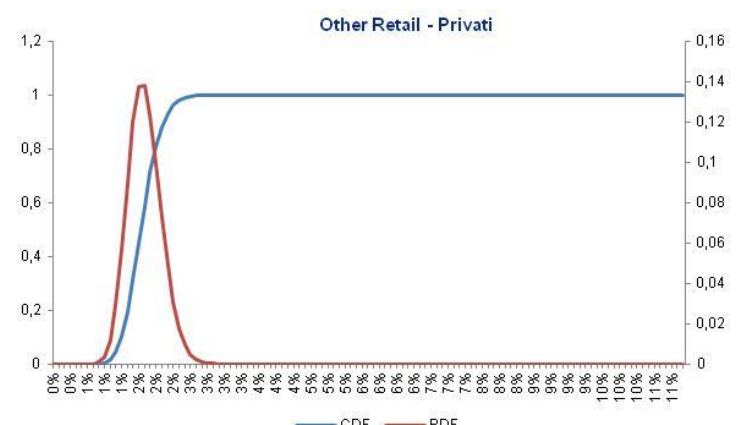

- $\mathrm{CDF}-\mathrm{PDF}$

Esposizioni verso o garantite da imprese - PMI

\begin{tabular}{|c|c|c|c|c|}
\hline & Dati Effettivi & LGD $45 \%$ & PD - 100bps & PD + 100bps \\
\hline $\mathrm{PD}$ & $3,72 \%$ & $3,72 \%$ & $2,72 \%$ & $4,72 \%$ \\
\hline LGD & $32,00 \%$ & $45,00 \%$ & $32,00 \%$ & $32,00 \%$ \\
\hline EL & $1,19 \%$ & $1,68 \%$ & $0,87 \%$ & $1,51 \%$ \\
\hline Corr Implicita & $3,20 \%$ & $1,67 \%$ & $4,91 \%$ & $2,30 \%$ \\
\hline Corr Basel & $10,93 \%$ & $10,93 \%$ & $12,14 \%$ & $10,20 \%$ \\
\hline UL Implicita & $2,67 \%$ & $2,43 \%$ & $2,98 \%$ & $2,49 \%$ \\
\hline UL Basel & $6,75 \%$ & $9,50 \%$ & $6,23 \%$ & $7,25 \%$ \\
\hline Delta UL & $4,08 \%$ & $7,07 \%$ & $3,25 \%$ & $4,75 \%$ \\
\hline
\end{tabular}

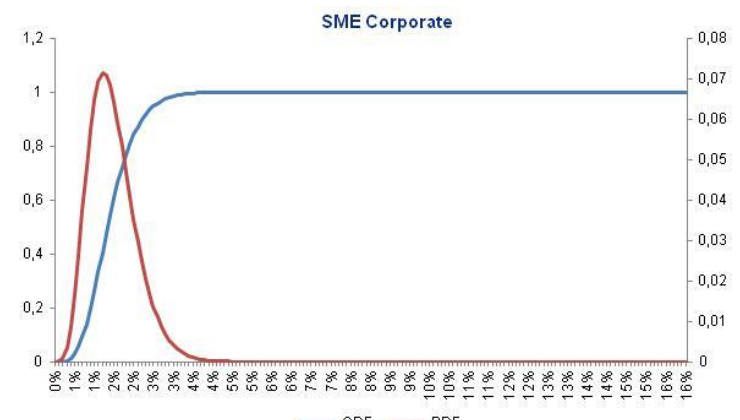

CDF $-\mathrm{PDF}$ 


\begin{tabular}{|ccccc|}
\multicolumn{5}{c}{ Esposizioni verso o garantite da imprese - Altre Imprese } \\
Dati Effettivi & LGD 45\% & PD - 50bps & PD + 50bps \\
\hline PD & $1,28 \%$ & $1,28 \%$ & $0,78 \%$ & $1,78 \%$ \\
LGD & $31,74 \%$ & $45,00 \%$ & $31,74 \%$ & $31,74 \%$ \\
EL & $0,41 \%$ & $0,57 \%$ & $0,25 \%$ & $0,564 \%$ \\
Corr Implicita & $5,84 \%$ & $3,15 \%$ & $10,75 \%$ & $3,74 \%$ \\
Corr Basel & $18,34 \%$ & $18,34 \%$ & $20,14 \%$ & $16,94 \%$ \\
UL Implicita & $2,08 \%$ & $1,81 \%$ & $2,62 \%$ & $1,82 \%$ \\
UL Basel & $6,00 \%$ & $8,51 \%$ & $5,02 \%$ & $6,64 \%$ \\
Delta UL & $\mathbf{3 , 9 2 \%}$ & $\mathbf{6 , 7 0 \%}$ & $\mathbf{2 , 4 0 \%}$ & $\mathbf{4 , 8 2 \%}$ \\
\hline
\end{tabular}

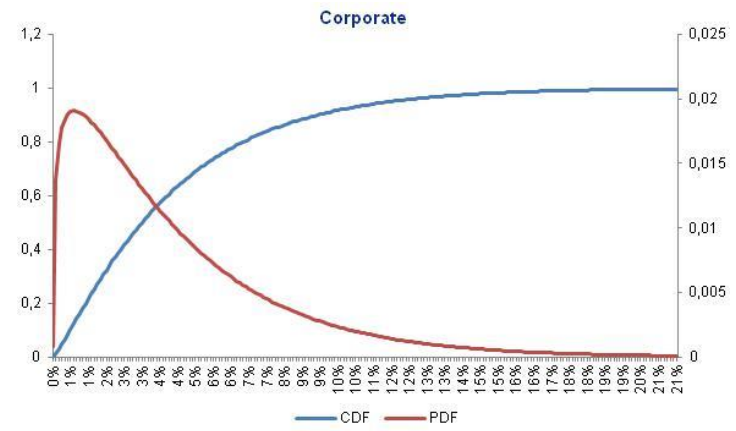

Occorre precisare che l'approccio prudenziale seguito dal Regolatore riflette anche la volontà di considerare un adeguato buffer che tenga in considerazione sia le assunzioni alla base del modello di Gordy (in primis quello di distribuzione normale delle perdite), sia la necessità di dover applicare le correlazioni a realtà molto differenti tra loro (sistemi bancari Giapponesi, Europei e Americani) e, pertanto, la "taratura" delle correlazioni è stata verosimilmente basata su valori più alti.

A fronte di questo, è necessario, tuttavia, evidenziare che la sostanziale sovrastima delle correlazioni ha determinato assorbimenti patrimoniali di molto superiori a quanto effettivamente utile per garantire la copertura dei rischi. Si ritiene che in sede di valutazione del rischio sistemico le Autorità di Vigilanza dovrebbero quantificare questo "excess" di patrimonio partendo dalle specificità dei diversi sistemi nazionali ed integrando le valutazioni del rischio sistemico (sia in sede SREP che in sede di esercizi di stress testing) al fine di considerare questo excess come una garanzia fornita dall'Ente al mercato per mitigare il rischio di contagio.

\subsection{Gli altri requisiti patrimoniali}

Per quanto attiene gli altri requisiti patrimoniali e di liquidità si è proceduto ad effettuare una valutazione qualitativa delle eventuali componenti che genererebbero un requisito patrimoniale o di liquidità eccessivamente oneroso rispetto al rischio sottostante:

\begin{tabular}{|c|c|c|c|c|}
\hline Richiesta Normativa & Normativa & Rischio & Tipo Requisito & Descrizione \\
\hline $\begin{array}{l}\text { Margine di conservatività } \\
\text { nella stima della } \text { CT di } \\
\text { ancoraggio }\end{array}$ & CRR & Credit Risk & Rwa & Il margine determina stime di PD maggiormente elevate \\
\hline $\begin{array}{l}\text { Floor allo } 0,03 \% \text { sulla PD } \\
\text { regolamentare }\end{array}$ & CRR & Credit Risk & Rwa & $\begin{array}{l}\text { Il floor sulla PD determina assorbimenti patrimoniali aggiuntivi } \\
\text { per le controparti migliori }\end{array}$ \\
\hline $\begin{array}{l}\text { Stima della LGD che } \\
\text { incorpori l'effetto downturn }\end{array}$ & CRR & Credit Risk & Shortfall & $\begin{array}{l}\text { La downturn determina un requisito patrimoniale sempre in } \\
\text { condizioni di stress test }\end{array}$ \\
\hline $\begin{array}{l}\text { Floor del } 10 \%-15 \% \text { sui } \\
\text { valori di LGD regolamentare } \\
\text { per i i mutui } \\
\text { residenziali/commerciali }\end{array}$ & CRR & Credit Risk & Shortfall & $\begin{array}{l}\text { Il floor dell'LGD determina assorbimenti patrimoniali } \\
\text { aggiuntivi per le controparti verso cui si hanno maggiori } \\
\text { garanzie }\end{array}$ \\
\hline AVC & CRR & Credit Risk & Rwa & $\begin{array}{l}\text { L'AVC determina un incremento dei requisiti patrimoniali verso } \\
\text { soggetti di grandi dimensioni del settore finanziario pari al } 25 \% \\
\text { a titolo di rischio sistemico. }\end{array}$ \\
\hline Beta & Art. 366 CRR & Market Risk & Rwa & $\begin{array}{l}\text { Il beta comporta la moltiplicazione del rischio effettivo per un } \\
\text { multiplo ad oggi pari ad almeno } 3 \text { volte il requisito. }\end{array}$ \\
\hline
\end{tabular}




\begin{tabular}{|c|c|c|c|c|}
\hline Richiesta Normativa & Normativa & Rischio & Tipo Requisito & Descrizione \\
\hline Stressed VaR & Art. 365 CRR & Market Risk & Rwa & $\begin{array}{l}\text { Lo stressed VaR oltre alla moltiplicazione del punto precedente } \\
\text { prevede un ulteriore double-counting del medesimo rischio }\end{array}$ \\
\hline Controparte & CRR & Counterparty Risk & Rwa & $\begin{array}{l}\text { Il Cva risk calcolato col metodo semplificato, sovrastima il } \\
\text { rischio rispetto ad un calcolo con un modello interno }\end{array}$ \\
\hline $\begin{array}{l}\text { Raccolta operational da } \\
\text { controparti wholesale }\end{array}$ & $\begin{array}{l}\text { CRR/Atto } \\
\text { Delegato }\end{array}$ & Liquidity Risk & LCR & $\begin{array}{l}\text { E' spesso impossibile identificare la quota parte di raccolta } \\
\text { effettivamente utilizzata per esclusive finalità operative (nel } \\
\text { qual caso poniamo prudentemente a zero la parte operational) }\end{array}$ \\
\hline $\begin{array}{l}\text { Raccolta da collocamento } \\
\text { certificates da classificare tra } \\
\text { strumenti derivati }\end{array}$ & BCBS & Liquidity Risk & NSFR & $\begin{array}{l}\text { La raccolta da certificates non solo non contribuisce al totale } \\
\text { ASF ma, al contrario, può richiedere finanziamento stabile in } \\
\text { quanto strumento derivato }\end{array}$ \\
\hline $\begin{array}{l}\text { Previsione di un ASF factor } \\
\text { dello } 0 \% \text { per le poste passive } \\
\text { senza scadenza predefinita }\end{array}$ & BCBS & Liquidity Risk & NSFR & 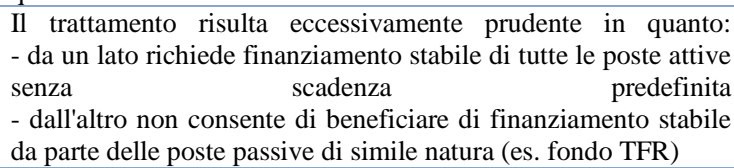 \\
\hline $\begin{array}{l}\text { Asimmetria } \quad \text { trattamento } \\
\text { derivati }\end{array}$ & BCBS & Liquidity risk & NSFR & $\begin{array}{l}\text { Il trattamento dei derivati è diverso a seconda del segno del } \\
\text { MTM: se positivo, non dà nessun contributo; se negativo, va } \\
\text { finanziato }\end{array}$ \\
\hline
\end{tabular}

Alle componenti sopra elencate si potrebbero aggiungere ulteriori elementi derivanti da normative in corso di discussione, come la Fundamental Review, che potrebbero generare ulteriori assorbimenti patrimoniali eccessivi rispetto ai rischi effettivamente detenuti:

\begin{tabular}{|c|c|c|c|c|c|}
\hline Richiesta Normativa & Normativ & & Rischio & Tipo Requisito & Descrizione \\
\hline Lambda & $\begin{array}{l}\text { Fundamental } \\
\text { review of } \\
\text { trading book }\end{array}$ & the & Market risk & Rwa & $\begin{array}{l}\text { Nell'ambito di un "Revised standardised approach", il Fuller } \\
\text { Risk Factor Approach prevede di mappare i singoli strumenti } \\
\text { in funzione di risk factor. L'Hedging può essere riconosciuto } \\
\text { come espressione di fattori di rischio sistematico (systematic } \\
\text { risk). }\end{array}$ \\
\hline Tutti i risk factor sottostanti & $\begin{array}{l}\text { Fundamental } \\
\text { review of } \\
\text { trading book }\end{array}$ & the & Market risk & Rwa & $\begin{array}{l}\text { Definizione di uno standardised framework comune in } \\
\text { funzione del quale stimare obbligatoriamente i requisiti di } \\
\text { capitale il quale possa costituire un indicatore di early } \\
\text { warning e, al tempo stesso, mitigare il rischi connessi al } \\
\text { sistema }\end{array}$ \\
\hline VaR based capital rules & $\begin{array}{l}\text { Fundamental } \\
\text { review of } \\
\text { trading book }\end{array}$ & the & Market risk & Rwa & $\begin{array}{l}\text { Le metodologie } \mathrm{VaR} \text { di stima dei requisiti di capitale hanno } \\
\text { mostrato assumere un andamento prociclico e non si osserva } \\
\text { un'adeguata gestione del rischio sistematico }\end{array}$ \\
\hline $\begin{array}{l}\text { Systematic factor } \mathrm{Z} \text { and } \\
\text { Rho(z) }\end{array}$ & $\begin{array}{l}\text { Fundamental } \\
\text { review of } \\
\text { trading book }\end{array}$ & the & Market risk & Rwa & $\begin{array}{l}\text { Nell'ambito della stima di P\&L di uno strumento finanziario, } \\
\text { attraverso il Partial Risk Factor approach, il rendimento di } \\
\text { uno strumento finanziario è espresso in funzione di un fattore } \\
\text { di sistema Z. }\end{array}$ \\
\hline Baseline correlations & $\begin{array}{l}\text { Fundamental } \\
\text { review of } \\
\text { trading book }\end{array}$ & the & Market risk & Rwa & $\begin{array}{l}\text { Nell'ambito della stima di P\&L di uno strumento finanziario, } \\
\text { attraverso il Partial Risk Factor approach, in un bucket di } \\
\text { strumenti finanziari, ciascuno di essi è correlato agli altri, in } \\
\text { quanto il rendimento di ogni strumento è correlato al fattore } \\
\text { sistemico } \mathrm{Z} \text { e individualmente tra di loro }\end{array}$ \\
\hline
\end{tabular}

\section{Considerazioni conclusive}

Le precedenti considerazioni portano a considerare che il rischio sistemico, pur essendo per sua natura macroprudenziale, è già stato abbondantemente indirizzato nell'attuali normative, attraverso una pluralità di interventi a carattere idiosincratico.

Ad un'attenta analisi possiamo dividere gli interventi in due macrocategorie che fanno riferimento a:

- interventi finalizzati a evitare l'insorgere di un evento sistemico potenzialmente in grado di generare una crisi sistemica. Si tratta di interventi che hanno l'obiettivo di ridurre le probabilità di un fallimento di una banca che, per dimensioni, interconnessione e altre caratteristiche si ritiene tale da poter generare in caso di fallimento, una crisi sistemica. E' questo il caso del G-SIB capital buffer;

- interventi finalizzati a rendere un evento sistemico meno probabile attraverso il rafforzamento delle capacità della singola banca di assorbire perdite o di resistere a situazioni di stress di liquidità.

Le misure della prima tipologia hanno l'obiettivo di ridurre la probabilità che un evento sistemico si verifichi. Le misure del secondo tipo hanno l'obiettivo di rafforzare la capacità della singola banca di resistere ad una crisi sistemica nel caso in cui venga innescata. In un certo senso, le prime sono misure di difesa "attiva", le seconde di difesa "passiva".

La nuova normativa di Vigilanza ha rafforzato gli strumenti pre-esistenti per la copertura dei rischi ed ha individuato ulteriori strumenti finalizzati a ridurre il rischio sistemico; sono state introdotte molte novità nei diversi ambiti, dai rischi di liquidità, al capitale, al ruolo dello SREP, e questo in un contesto di armonizzazione delle normative europee volto a cogliere l'evoluzione dei mercati finanziari sempre più integrati e globali. 
Alla base delle scelte adottate dai Regolatori, è tuttavia possibile affermare che il criterio che maggiormente ha inciso sulla selezione degli strumenti, sulle finalità sulle quali si basano, nonché sui loro meccanismi di attivazione, è stato guidato dalla necessità/volontà di dotare le banche di maggiori dotazioni patrimoniali secondo l'assioma "maggior patrimonio = minor rischio sistemico".

Da un punto di vista economico, non è possibile ridurre a zero la probabilità di insolvenza di una banca, anche di una G-SIB, quindi il rischio sistemico non è eliminabile. Certamente, l'incremento della loss absorption capability da parte delle G-SIB rappresenta un caposaldo della mitigazione del rischio sistemico, dato il presupposto che il fallimento di una G-SIB può innescare una crisi sistemica. In quest'ottica, il prospettato ulteriore rafforzamento della capacità di assorbire perdite attraverso l'introduzione del TLAC per le G-SIB rappresenta un indubbio passo nella direzione auspicata di ridurre le probabilità di fallimento, ma non potrà mai impedirlo. Pertanto, la strada di richiedere continui aumenti delle dotazioni di capitale alle G-SIB non è perseguibile all'infinito.

Per le altre banche, che sono soggetti "passivi" nei confronti del rischio sistemico, il rafforzamento di capitale e di liquidità rappresenta anch'esso un passo nella giusta direzione; anche in questo caso, poiché lo svilupparsi degli eventi non è prevedibile ex ante e le caratteristiche di una crisi sistemica sono spesso di magnitudo talmente elevata da non essere realmente mitigabili, non è percorribile la strada di un continuo e generalizzato aumento delle dotazioni di capitale, perseguite spesso attraverso add-on o misure più restrittive di misurazione del capitale richiesti a fronte dei singoli rischi. Ulteriori incrementi potrebbero giustificarsi solo alla luce di una dimostrata evidenza empirica dell'inadeguatezza delle misure interne o standardizzate richieste (come è stato dimostrato per i rischi di mercato, di controparte e di liquidità nel framework Basilea 2).

Va inoltre considerato che la stabilità del sistema passa anche - e forse soprattutto - attraverso la capacità delle banche di generare organicamente il capitale necessario a sostenere il proprio business. E' questo il principio alla base della Business Model Analysis, che costituisce uno dei quattro pilastri del nuovo modello di supervisione (SREP). Un eccessivo appesantimento dei requisiti di capitale, datele condizioni di mercato e il business model tipico delle banche italiane, renderebbe sempre più complicato remunerare adeguatamente il capitale, generando instabilità e aumentando la probabilità se non fallimenti certamente di situazioni di crisi a meno di non dover compensare i rendimenti attraverso modelli di business e attività ad alto rischio, ottenendo quindi il risultato opposto a quello desiderato.

Il regolatore - e il supervisore - dovranno quindi valutare il trade off tra l'aumento dei requisiti di capitale a parità di rischi, e il rischio generato da una cronica incapacità di generare utili adeguati, col rischio di un moral hazard verso attività con un miglior rapporto rendimento/capitale, e quindi più rischiose.

Le evidenze della sovrastima dei requisiti patrimoniali, a nostro giudizio, rimettono in discussione l'assioma maggior patrimonio $=$ minor rischio sistemico e porterebbero a ritenere che il patrimonio possa rappresentare solo uno dei presidi a fronte del rischio e che l'eccesso di patrimonio non possa costituire un ulteriore presidio, ma, al più, comporterebbe una maggiore difficoltà nel reperire capitale e nel remunerarlo, determinando non un potenziamento della patrimonializzazione delle Banche, ma un ricorso al deleveraging o alla rimodulazione dell'asset allocation bancaria, tipicamente creditizia, verso strumenti di mercato del banking book assoggettate a requisiti patrimoniali meno stringenti.

Fabio Salis; Pietro Penza; Pasquale Costa; Nicola Mariano; Ester Inserra Imparato; Ruggiero Gorgoglione; Francesco D'Avanzo; Camillo Gilberto; Marco Rocchi; Andrea Consonni; Agostino Grua; Francesco Mazza; Claudio Barberi; Antonio Castagna); Giorgio Canobbio; Marco Salemi 


\section{Bibliogafia}

- $\quad$ Bank of International settlment - P.Clement - Origini ed evoluzione del termine macroprudenziale

- Basel Committee on Banking Supervision - Basel II: International Convergence of Capital Measurement and Capital Standards: A Revised Framework

- Basel Committee on Banking Supervision - Basilea 3 - Schema di regolamentazione internazionale per il rafforzamento delle banche e dei sistemi bancari

- Basel Committee on Banking Supervision - Global systemically important banks: updated assessment methodology and the higher loss absorbency requirement

- Basel Committee on Banking Supervision - Proposal to ensure the loss absorbency of regulatory capital at the point of nonviability

- Basel Committee on Banking Supervision - An Explanatory Note on the Basel II IRB Risk Weight Functions

- European Central Bank - Systemic Risk a Survey

- European Central Bank - Special Feature A for a discussion of the use of stress tests during a crisis

- $\quad$ European Central Bank - Predicting Distress in European Banks

- European Central Bank - CISS - A Composite Indicator of Systemic Stress in the Financial System

- European Central Bank - A macro stress testing framework for assessing systemic risks in the banking sector

- European Central Bank - Systemic risk diagnostics coincident indicators and early warning signals

- European Central Bank - Macro-financial vulnerabilities and future financial stress assessing systemic risks and predicting systemic events

- $\quad$ ESRB - The ESRB Handbook on Operationalising Macro-prudential Policy in the Banking Sector

- $\quad$ ESRB - A review of macro-prudential policy in the EU one year after the introduction of the CRD/CRR

- $\quad$ ESRB - ESRB Recommendation on the macro-prudential mandate of national authorities

- ESRB - Principles for the development of a macro-prudential framework in the EU in the context of the capital requirements legislation

- $\quad$ ESRB - Raccomandazione -sugli obiettivi intermedi e gli strumenti di politica macroprudenziale

- $\quad$ ESRB - The ESRB and national macro-prudential measures - its role and first experiences

- $\quad$ EBA - Guidelines on common procedures and methodologies for the supervisory review and evaluation process (SREP)

- EBA-GL-2015-02 GL on recovery plan indicators

- $\quad$ EBA-GL-2015-03 Guidelines on Early Intervention Triggers

- $\quad$ EBA-GL-2015-07+GL+on+failing+or+likely+to+fail

- $\quad$ EBA-RTS-2014-15 (Final draft RTS on Resolution Plan Contents)

- $\quad$ EBA-RTS-2015-05 RTS on MREL Criteria

- $\quad$ EBA - GDL - assessment of other systemically important institutions O-SIIs

- $\quad$ EBA - RTS - Art.131 CRD - global systemically important institutions

- EBA-Op-2014-02_Opinion_on_measures_to_address_macroprudential_or_systemic_risk

- $\quad$ Fitch Rating - Basel II Correlation Values - An Empirical Analysis of EL, UL and the IRB Model

- $\quad$ Michel B. Gordy - A Risk-Factor Model Foundation for Ratings-Based Bank Capital Rules

- Direttiva 2013/36/UE del parlamento europeo e del consiglio del 26 giugno 2013 sull'accesso all'attività degli enti creditizi e sulla vigilanza prudenziale sugli enti creditizi e sulle imprese di investimento, che modifica la direttiva 2002/87/CE e abroga le direttive 2006/48/CE e 2006/49/CE

- Regolamento (UE) N. 575/2013 del parlamento europeo e del consiglio del 26 giugno 2013 relativo ai requisiti prudenziali per gli enti creditizi e le imprese di investimento e che modifica il regolamento (UE) n. 648/2012

- Direttiva 2014/59/UE del parlamento europeo e del CONSIGLIO del 15 maggio 2014 che istituisce un quadro di risanamento e risoluzione degli enti creditizi e delle imprese di investimento e che modifica la direttiva 82/891/CEE del Consiglio, e le direttive 2001/24/CE, 2002/47/CE, 2004/25/CE, 2005/56/CE, 2007/36/CE, 2011/35/UE, 2012/30/UE e 2013/36/UE e i regolamenti (UE) n. 1093/2010 e (UE) n. 648/2012, del Parlamento europeo e del Consiglio 


\title{
Linee guida sulla prudent valuation ${ }^{1}$ \\ di Marco Bianchetti ( Intesa San Paolo), Umberto Cherubini (Università di Bologna) e Roberto Tubaldi (Swiss Finance Institute)
}

\begin{abstract}
The EU Capital Requirement Regulation (CRR) [1] and EBA Regulatory Technical Standard for prudent valuation [2], published on Jan. 1st, 2014 and Jan. 28th, 2016, respectively, constitute the EU Prudent Valuation Framework. The CRR, art. 34, requires to Institutions a prudent valuation of positions measured at fair value and the deduction of the resulting Additional Valuation Adjustments (AVAs) from the Common Equity Tier One (CET1) capital. The art. 105 disciplines the AVAs intended to achieve an appropriate level of certainty in prudent value. The EBA RTS [14] allow two approaches to prudent valuation. The simplified approach, applicable by small financial institutions (with total absolute fair-valued assets and liabilities below EUR 15 billions), prescribes a very simple total AVA equal to $0.1 \%$ of the total fair value. The core approach, compulsory for institutions above the EUR 15 billion threshold, prescribes the calculation of 9 AVAs, referring to different sources of valuation uncertainty, as the excess of valuation adjustments required to achieve the prudent value with $90 \%$ level of confidence. Five out of nine AVAs include a 50\% weight to take diversification into account and avoid double counting effects. Those positions for which a change in their fair value affects only partially the CET1 may be partially excluded from the AVAs calculation.
\end{abstract}

The EU Prudent Valuation Framework represents a challenge for financial institutions, both from a methodological and practical point of view. In this work, we seek an ambitious fourfold result. First, we work out a practical realization of the regulatory requests, smoothing the difficulties arising from the detailed interpretation of the rules. Second, we give a comprehensive methodological analysis of the different sources of valuation risk (ambiguity, liquidity, correlation, concentration and model risks) that bring about the need of computing valuation adjustments. Third, we explore a wide range of possible individual AVA calculation approaches, providing many practical case studies mimicking the actual valuation scenarios that Institutions might face. Finally, we design a comprehensive example of the entire Prudent Valuation process, covering both organizational and IT issues.

In conclusion, this work provides a set of guidelines and sound practices that could be used as a starting point and, possibly, a benchmark for Prudent Valuation. On one side, Institutions will benefit of the rules explained and applied into a variety of case studies. On the other side, regulators will benefit to see how the rules are interpreted and applied by Institutions. These guidelines do not aim either to give any authentic interpretation of the regulations, which ultimately belongs to its corresponding owners (EU and EBA), or to question the principles behind the regulations, since real challenges may come only from practical applications of the principles to real financial portfolios, and comparisons across different financial institutions.

\section{Introduzione}

La Capital Requirement Regulation (CRR) [1] dell'Unione Europea e i Regulatory Technical Standards (RTS) per la prudent valuation [2] dell'EBA, pubblicati rispettivamente l'1 Gennaio 2014 e il 28 Gennaio 2016, costituiscono la cornice europea alla regolamentazione della Prudent Valuation. L'essenza della normativa va ricercata nel combinato disposto degli artt. 34 e 105 della CRR. Da un lato, l'articolo 34 richiede agli istituti finanziari di provvedere alla valutazione prudenziale delle posizioni misurate al fair value e la deduzione dal capitale di tipo Common Equity Tier One (CET1) di un insieme di Additional Valuation Adjustment (AVA) che rappresentano possibili fonti di incertezza valutativa; dall'altro, l'art. 105 disciplina gli AVA, con lo scopo di raggiungere un certo livello di accuratezza nel calcolo del prudent value. Tale analisi delle fonti di incertezza valutativa costituisce il cosiddetto core approach, obbligatorio per gli istituti il cui valore assoluto di attività e passività calcolate al fair value e rientranti nel perimetro di applicazione della normativa supera i 15 miliardi. Gli istituti che non rientrano in questa categoria possono applicare il simplified approach, che prescrive semplicemente di calcolare l'AVA totale come lo $0,1 \%$ del fair value totale. La definizione del perimetro di applicazione è cambiata progressivamente nel corso della definizione dell'assetto regolamentare e rimanda a considerazioni tecniche piuttosto complesse.

Le ragioni economiche di questo intervento regolamentare derivano dalla rilevanza che il rischio valutativo ha assunto nella recente crisi. In primo luogo, infatti, gli aspetti di incertezza sul contenuto informativo dei prezzi di mercato (Market Price Uncertainty, MPU), sui costi di chiusura di una posizione (Close-out Cost, CoCo) e sui modelli utilizzati per la valutazione di prodotti non lineari (Model Risk, MORI) risultano accentuati in periodi di particolare tensione e di scarsa liquidità dei mercati. In secondo luogo, negli ultimi anni il processo valutativo è diventato progressivamente più sofisticato. Oggi, la valutazione di un prodotto finanziario è un processo composito che tiene in considerazione non solo i principali fattori di rischio intrinsechi

${ }^{1}$ Il presente articolo rappresenta una sintesi del lavoro della Commissione "Prudent Valuation", pubblicato sul sito di AIFIRM $\underline{w w w . a i f i r m . i t}$ 
dello strumento, ma anche di quei fattori addizionali che possono avere un impatto nella valutazione, quali il rischio di default della controparte, il funding risk, le garanzie, il costo del capitale, etc.

Tali fattori addizionali sono generalmente racchiusi in un'unica categoria denominata X-Valuation Adjustment (XVA) e in generale rappresentano un elemento di non linearità nella valutazione. Data questa non linearità e il fatto di essere in larga misura legati a rischi non direttamente replicabili sui mercati, questi elementi di aggiustamento sono essi stessi esposti a problemi di incertezza valutativa, sia per l'opacità del prezzo e del valore di chiusura, sia per il rischio di modello. Il fine di questo articolo è quello riportare i tratti essenziali del problema del rischio valutativo, sintetizzando il lavoro della Commissione AIFIRM sul tema. Maggiori approfondimenti possono essere ricavati dal rapporto stilato a conclusione dei lavori di tale commissione.

\section{La "prudent valuation" come risposta al rischio contabile durante le crisi}

La regolamentazione sulla "prudent valuation" nasce come risposta alla necessità di far fronte al rischio contabile che emerge nei periodi di stress, quando il concetto di fair value diventa opaco e difficile da applicare a causa dell'eccessivo "rumore" presente nei valori di mercato. Sotto questo profilo, il nuovo approccio europeo sulla Prudent Valuation rappresenta una sfida per gli istituti finanziari di grandi dimensioni, sia da un punto di vista metodologico che pratico. Per capire la motivazione all'origine della normativa europea in materia di valutazione prudenziale, occorre analizzare uno dei problemi principali della crisi dei "subprime" del 2008. In questa occasione, il tema della valutazione degli strumenti finanziari e il concetto di fair value sono stati messi alla prova, per la prima volta, in una situazione di crisi sistemica. Si noti che il concetto di fair value era stato introdotto nei principi contabili internazionali con l'obiettivo di avvicinare il più possibile i valori registrati in bilancio e i prezzi osservati nei mercati. Tuttavia, questo principio non ha superato la prova della crisi e proprio il sistema contabile si è rivelato uno dei maggiori fattori di contagio. Il problema è stato che, quando la liquidità degli strumenti finanziari si esaurisce, anche il concetto di fair value, basato sugli exit price osservati in normali transazioni tra investitori, mostra i suoi limiti. In assenza di liquidità, dunque, occorre distinguere tra gli effetti dovuti ai movimenti nei valori fondamentali degli strumenti e gli effetti derivanti dallo stato del mercato, che possono rivelarsi transitori. È stato proprio questo meccanismo a far sì che, durante la crisi dei mutui "subprime", grandi istituti finanziari andassero in "default", non per aver registrato perdite non nei suddetti mutui, ma per il crollo del fair value delle attività presenti nel loro book di negoziazione. La ragione di ciò risiede nel fatto che nei periodi critici i prezzi sono più vicini a valori di saldo, che in un normale giorno di negoziazione. Condizione necessaria per il rischio contabile sopra descritto è la mancanza di liquidità, qui intesa non come perdita materiale nella liquidazione di una posizione, ma come impatto che la prospettiva di questi prezzi di liquidazione può avere sui prezzi. Questa diversa concezione di liquidità fa sì che l'effetto sia indiretto, da un lato, ma ancora più intenso, dall'altro. È indiretto perché non si tratta di illiquidità strettamente legata al così detto "exit price", ma al modo in cui tale prezzo è registrato nella valutazione della posizione. Inoltre, l'effetto è ancora più forte perché dipende dal livello di liquidità e trasparenza del mercato nel suo insieme, nel senso che va tenuto presente non solo il mercato dove il prodotto è scambiato, ma anche informazioni di mercato di tipo generale, quali la struttura a termine dei tassi di interesse, la volatilità implicita e così via.

Tuttavia, definire e misurare la liquidità è un compito assai arduo e mostra aspetti generalmente qualitativi, in particolare quando si vuole analizzare il legame tra liquidità e trasparenza. È necessario chiedersi per quale livello di liquidità i prezzi siano pienamente informativi e quanti attori di mercato occorrano per assicurare un efficiente processo di formazione del prezzo (price discovery). Rispondere a domande su come l'informazione viene incorporata nei prezzi delle attività finanziarie e riportata in bilancio non è mai semplice, specialmente se si considerano le non sempre limpide relazioni tra banche e autorità contabili. In maniera più esplicita, da un lato una banca può essere tentata di definire illiquido un mercato per posticipare il riconoscimento di perdite, mentre dall'altro molte banche, in passato, sono state forzate a riconoscere delle perdite apparenti, relative a prezzi completamente privi di significato.

L'idea di introdurre la Prudent Valuation nasce proprio dalla necessità di assicurare il sistema bancario contro il rischio di valutazione che emerge nelle situazioni sopra descritte. Il principio di fondo è quello di prevedere capitale regolamentare che tenga in considerazione le valutazioni soggette ad incertezza valutativa. Tale principio si riferisce, in modo particolare, alle attività presenti nel "trading book", ma va comunque applicato a tutte le posizioni misurate al fair value e soggette a rischio di valutazione. E' importante distinguere il rischio di valutazione dal rischio di mercato. La differenza è che una perdita derivante dal rischio di mercato viene registrata nel caso in cui uno scenario avverso può materializzarsi in futuro, mentre una perdita legata al rischio di valutazione è tenuta in considerazione nel momento in cui alcuni fattori di rischio non sono incorporati nel prezzo corrente o non possono essere valutati con accuratezza. In termini tecnici, si tratta della stessa differenza tra rischio e incertezza che viene fatta risalire agli anni 20 del secolo scorso e attribuita a Frank Knight. Si definisce rischio il caso in cui siano conosciuti i fattori di rischio e le loro probabilità, e si parla di incertezza quando queste probabilità non sono note con precisione. E' questa forma di incertezza (nota nella letteratura scientifica come "incertezza in senso di Knight") che dà origine al rischio di valutazione e alla necessità di una valutazione conservativa ("prudent valuation").

Per quanto riguarda la teoria della finanza, il concetto di "prudent valuation" richiama come condizione necessaria il concetto dei mercati incompleti. L'argomento è che, in un mercato completo, ogni prodotto finanziario può essere associato ad una strategia di replica costruita su strumenti perfettamente liquidi, da cui deriva un prezzo unico. Per questo motivo, non ci sarebbe spazio per il rischio di valutazione se il mercato fosse completo. Tuttavia, nella realtà i mercati sono strutturalmente incompleti e il portafoglio di replica è soggetto a errore (hedging error). In altri termini, non esistono portafogli di replica di titoli perfettamente liquidi che garantiscano con sicurezza lo stesso risultato di un prodotto finanziario. È la presenza di questo margine di "errore di copertura" che lascia spazio al calcolo di valori più conservativi, come quelli richiesti nella "prudent valuation". 


\section{La storia della prudent valuation}
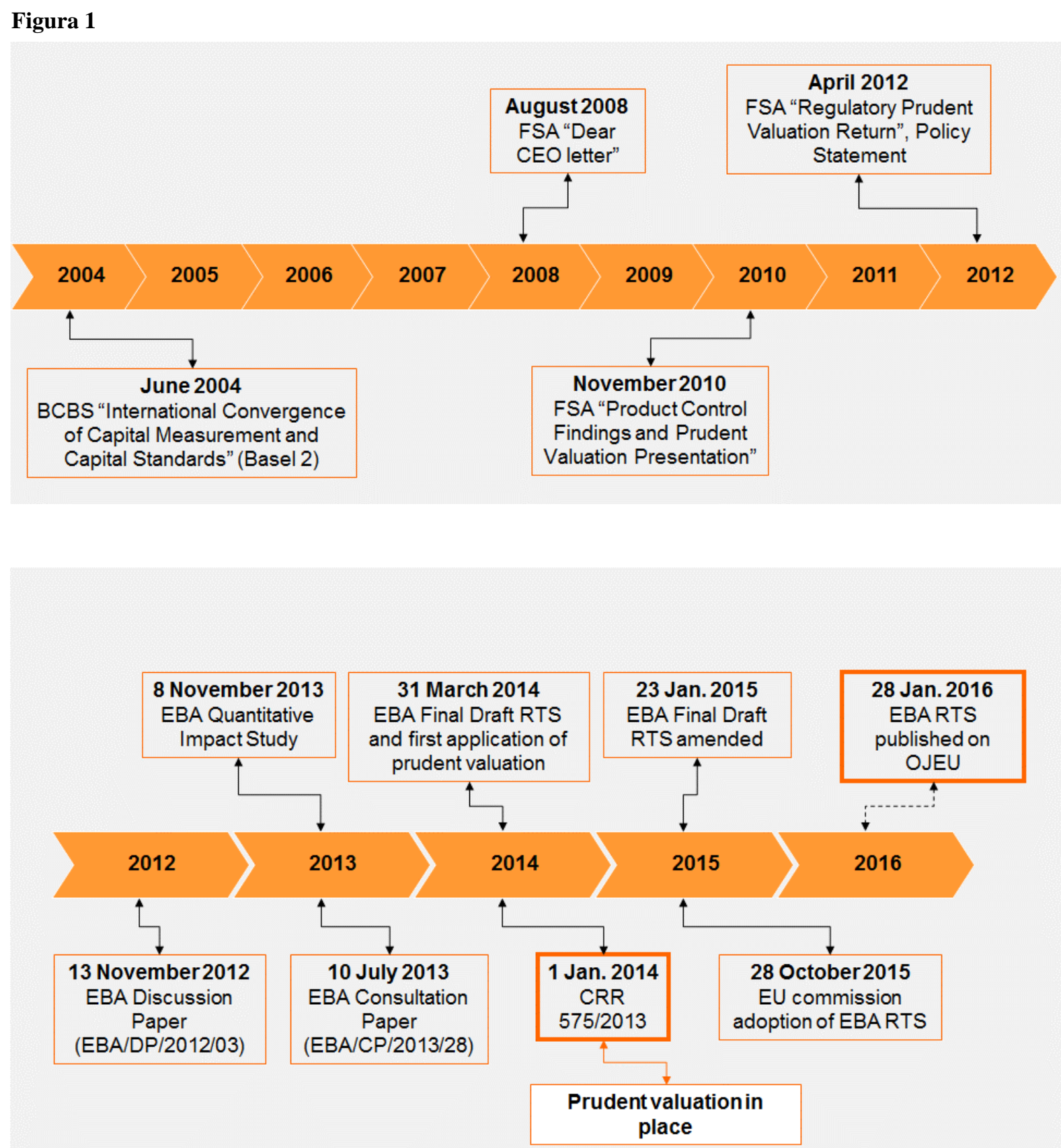

\section{Figura 1: La storia della prudent valuation}

La storia della "prudent valuation" (le cui date fondamentali sono riportate in Figura (1) inizia ben prima della crisi. Già nel 2004, il regolamento noto come Basilea II [3] si occupava dei problemi relativi alla "prudent valuation" nella sezione riguardante il book di negoziazione, con particolare riferimento a sistemi di controllo, metodi di valutazione e aggiustamenti alla valutazione. Negli anni successivi, la prima normativa completa sulla "prudent valuation" viene realizzata in Gran Bretagna dalla Financial Stability Agency (FSA) durante la crisi. Iniziato con una famosa lettera dei banchieri britannici sul tema, il processo normativo si è concluso con la redazione del regolamento vero e proprio nel 2012 (vedi rif. [4][5][6]). Alla fine dello stesso anno il processo normativo inizia anche in Unione Europea attraverso i diversi consultation paper dell'Autorità Bancaria Europea (vedi rif. [7]-[10]) e l'approvazione della CRR. Tutte queste diverse normative condividono lo stesso principio di fondo: le posizioni presenti nel book di negoziazione vanno valutate tenendo presenti ipotesi conservative e la differenza di prezzo va coperta attraverso l'accantonamento di un ammontare adeguato di capitale regolamentare. 


\section{I requisiti prudenziali definiti dall'EBA}

Come accennato nell'introduzione, la normativa sulla "prudent valuation" e sul calcolo degli AVA si può riassumere nel combinato disposto degli artt. 34 e 105 della CRR. Di maggiore importanza, ai fini dell'applicazione della normativa, sono gli standard tecnici (RTS) definiti dall'EBA [2]. A caratterizzare gli EBA RTS è la divisione tra "simplified" e il "core approach", descritta in precedenza. Per quanto riguarda il "core approach", l'AVA totale è una combinazione dei nove AVA descritti nella CRR all'art.105.10-11:

- Market price Uncertainty (MPU)

- Close out Costs (CoCo)

- Model risk (MoRi)

- Unearned credit spread (UCS)

- Investing and funding costs (IFC)

- Concentrated positions (CoPo)

- Future administrative costs (FAC)

- Early termination (EaT)

- Operational risks (OpR)

Ogni AVA è calcolato come differenza tra l'aggiustamento necessario a raggiungere una valutazione prudenziale, data l'esposizione al relativo rischio di valutazione della posizione, e il fair value della posizione stessa, includendo ogni altra possibile correzione già prevista dagli standard contabili.

Dove possibile, il valore della posizione è connesso ad uno spettro di possibili valori e ad uno specifico livello di confidenza (90\%). In tutti gli altri casi, si applica un approccio "expert-based", tenendo conto dei principali fattori di rischio. Anche in questo caso, il livello di confidenza è fissato al $90 \%$.

Agli AVA MPU, CoCo e MoRi, relativi ad una singola valutazione, è attribuito un peso del $50 \%$. Il peso tiene conto delle sovrapposizioni tra AVA ed è inteso ad evitare valutazioni eccessivamente prudenziali (diversification benefit). Gli AVA UCS e IFC, che riguardano gli aggiustamenti per rischio di controparte e costi di finanziamento, sono considerati compositi e quindi suddivisi nelle loro componenti di rischio valutativo elementari: MPU, CoCo e MoRi. I valori di questi AVA vengono poi aggregati agli AVA MPU, CoCo e MoRi corrispondenti. Per quanto riguarda, infine, gli AVA CoPo, FAC, EaT e OpR, il peso attribuito è del 100\%. L'AVA totale è poi calcolato come somma degli AVA individuali.

Nell'ambito del "core approach", quando l'istituto non è in grado di calcolare il singolo AVA per un qualche strumento finanziario, gli EBA RTS suggeriscono una soluzione "fall-back" (art. 7.2b degli EBA RTS), che calcola direttamente l'AVA come somma del $100 \%$ dei profitti netti non realizzati dallo strumento, il $10 \%$ del valore nominale nel caso di strumenti derivati e il $25 \%$ del valore assoluto della differenza tra fair value e $100 \%$ dei profitti netti non realizzati per gli altri prodotti. Tale soluzione è chiaramente dannosa in termini di capitale e, per questo, dovrebbe venire usata, eccezionalmente, solo in ultima istanza.

Infine, la sezione 4, artt. 18-19, degli EBA RTS definisce ulteriori requisiti in termini di documentazione (art. 18), sistemi e controllo (art. 19). Tali articoli richiedono essenzialmente agli istituti di dare attuazione ad una politica interna in due livelli: una per il fair value (Fair Value Policy) e una per il prudent value (Prudent Valuation Policy). La Figura 2 fornisce una rappresentazione di alto livello delle regole per la "prudent valuation" presenti negli EBA RTS. 


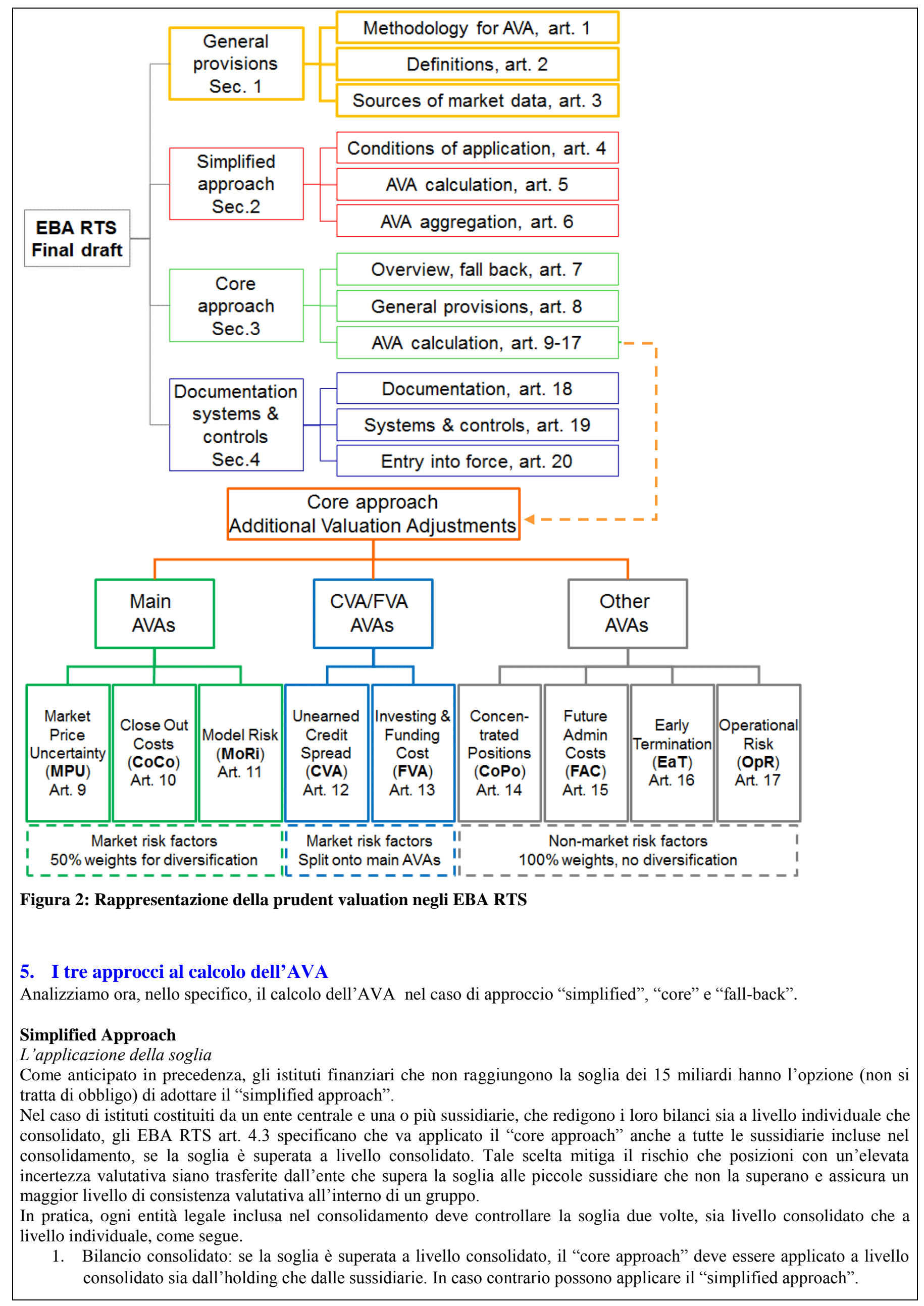


2. Bilancio individuale: se la soglia viene superata, la singola entità legale deve adottare il "core approach". In caso contrario può adottare il "simplified approach".

Calcolo dell'AVA

Gli EBA RTS, artt. 5-6, suggeriscono che, in caso di "simplified approach", l'AVA totale è calcolato come lo $0,1 \%$ del valore assoluto aggregato delle posizioni misurate al fair value. Ciò può essere schematizzato nella seguente formula,

$$
A V A(t)=0.1 \% \times\left[F V_{\text {Assets }}+\left|F V_{\text {Liabilities }}\right|\right]
$$

dove

$$
\begin{aligned}
& F V_{\text {Assets }}:=\sum_{\substack{i=1 \\
\text { Neistilities }}}^{\mathbb{N}_{\text {Assgts }}} F V_{i}(t), \\
& F V_{\text {Liabilities }}:=\sum_{i=1}^{\mathbb{N}_{\text {Liabilitivs }}} F V_{i}(t) \text {. }
\end{aligned}
$$

Nella Tabella 1 viene mostrato un esempio di calcolo dell'AVA seguendo il simplified approach.

Simplified approach

\begin{tabular}{lrrrr}
\hline \multicolumn{1}{c}{ Instrument } & Fair value & $\begin{array}{c}\text { Accounting } \\
\text { reserve }\end{array}$ & $\begin{array}{c}\text { Prudential } \\
\text { filter }\end{array}$ & $\begin{array}{c}\text { Fair Value } \\
\text { for AVA }\end{array}$ \\
\hline Derivatives HFT BTB & $14,000.00$ & 0.00 & $0 \%$ & 0.00 \\
Derivatives HFT & $5,000.00$ & 0.00 & $100 \%$ & $5,000.00$ \\
Bonds AFS & $12,000.00$ & -120.00 & $20 \%$ & $2,400.00$ \\
FV Option & $5,000.00$ & 0.00 & $100 \%$ & $5,000.00$ \\
Bonds AFS EU govies & $2,000.00$ & -4.00 & $0 \%$ & 0.00 \\
O.I.C.R & $1,000.00$ & 0.00 & $100 \%$ & $1,000.00$ \\
\hline Total portfolio & $\mathbf{3 9 , 0 0 0 . 0 0}$ & $-\mathbf{1 2 4 . 0 0}$ & & $\mathbf{1 3 , 4 0 0 . 0 0}$ \\
\hline Data in $€ /$ mln & & \multicolumn{1}{c}{ AVA } & 13.400 \\
\cline { 3 - 5 }
\end{tabular}

Tabella 1: Esempio di calcolo dell'AVA nell'ambito del simplified approach. I dati non si riferiscono a portafogli reali.

Passaggio dal "simplified" al "core approach"

L'art.4.4 degli EBA RTS disciplina le condizioni del passaggio dal "simplified" al "core approach": nel caso in cui gli istituti che applicano il "simplified approach" superino la soglia di €15 miliardi per due quadrimestri consecutivi, sono tenuti a darne notifica all'autorità competente e progettare un piano per il passaggio al "core approach" nell'arco dei due successivi quadrimestri. Data tale disposizione, le linee guida AIFIRM suggeriscono il seguente albero decisionale.

1. Nel caso in cui l'istituto che applica il "simplified approach" superi la soglia di $€ 15$ miliardi:

a. in due quadrimestri non consecutivi, può mantenere il "simplified approach";

b. per due quadrimestri consecutivi, deve passare al "core approach" entro i due quadrimestri successivi.

2. Nel caso in cui l'istituto che applica il "core approach" smetta di superare la sogli di €15 miliardi, può passare al "simplified approach" per quel quadrimestre. Nel caso di passaggio al "simplified", durante i quadrimestri successivi, vanno applicate le condizioni di cui al punto 1 . Chiaramente, in questo caso, l'istituto considererà il valore totale dell'AVA con il "simplified" e il "core approach" e valuterà le possibili opportunità di riduzione delle riserve di capitale.

\section{Core Approach}

Per quanto riguarda il "core approach", gli EBA RTS suggeriscono diversi approcci al calcolo dei differenti gruppi di AVA. In particolare, per il primo gruppo (AVA MPU, CoCo, MoRi) vengono suggerite due metodologie di calcolo (EBA RTS Annex 1 e sezioni 8.4-8.5 degli RTS):

\section{- Metodo \#1}

Il primo metodo può essere descritto come segue. Dato il fair value $F V_{i}^{(t)}$ di una posizione $p_{\mathrm{i}}$ e il suo corrispondente valore prudenziale $P V_{i j k}(t)$ della categoria $\mathrm{k}$ associata all'incertezza valutativa $u_{j}$, gli AVA calcolati secondo il core approach sono definiti come 


$$
\begin{aligned}
A P V A\left(t, p_{i}, u_{j}, k\right): & =0.5\left[F V\left(t, p_{\mathrm{i}}\right)-P V\left(t, p_{\mathrm{i}}, u_{j}, k\right)\right], \\
\operatorname{AVA}(t, k) & :=\sum_{i=1}^{N_{D}} \sum_{j=1}^{\mathbb{N}_{u}} A P V A\left(t, p_{\mathrm{i}}, u_{j}, k\right),
\end{aligned}
$$

dove:

- $A P V A_{\mathrm{ijk}}(t):=\operatorname{APVA}\left(t_{,}, p_{\mathrm{i}}, u_{j_{j}}, k\right)$ e il $k$-esimo AVA della posizione $p_{\mathrm{i}}$ rispetto all'incertezza valutativa $u_{j}$ al tempo $t$, pesato ai fini di aggregare tutti gli AVA;

- $A V A_{k}(t)$ è l'AVA totale della k-esima categoria associata a tutte le fonti di rischio rilevanti $u_{1, n,}, u_{\mathbb{N}_{u}}$ e alle posizioni $p_{1}, \ldots, p_{N_{p}}$.

Si noti che:

- $A V A_{k}(t)$ include sempre ad ogni livello (valutazione dell'esposizione, AVA totale, prudent valuation adjustment (PVA) totale) il peso di aggregazione 0.5 ;

- $A V A_{k}(t) \geq 0 \forall k$ ad ogni livello (valutazione dell'esposizione, AVA totale, PVA totale), sia pre che post aggregazione;

- $A V A_{k}(t)=0$ quando il fair value è di per sé prudenziale con riferimento alla fonte di rischio $A V A_{j}$, $F V_{i}(t)=P V_{i j k}(t)$;

- la precedente equazione vale sia per le attività $(F V(t)>0)$, che per le passività $(F V(t)<0)$.

Per quanto riguarda i derivati, si ricorda che il valore totale può essere suddiviso tra le diverse componenti di rischio. Si fa l'ipotesi che tali componenti non siano strettamente correlate. In particolare, si ipotizza che il valore di mercato non sia strettamente correlato con il credit and funding risk. In questo caso, anche i risultati degli AVA vanno suddivisi nelle loro componenti

$$
A P V A\left(t, p_{i}, u_{j}, k\right):=A P V A_{0}\left(t, p_{i}, u_{j}, k\right)+A P V A\left(t, p_{i}, u_{j}, C V A\right)+A P V A\left(t, p_{i}, u_{j}, F V A\right)+\cdots
$$

\section{- Metodo \#2}

Il secondo metodo è descritto dalla seguente formula:

$$
\begin{gathered}
\operatorname{APVA}\left(t, p_{\mathrm{i}}, u_{j}, k\right):=\max \left\{0,\left[F V\left(t, p_{\mathrm{i}}\right)-P V\left(t, p_{\mathrm{i}}, u_{j}, k\right)\right]-0.5\left[E V\left(t, p_{\mathrm{i}}\right)-P V\left(t, p_{\mathrm{i}}, u_{j}, k\right)\right]\right\} \\
A V A(t, k):=\sum_{\mathrm{i}=1}^{N_{D}} \sum_{j=1}^{N_{u}} \operatorname{APVA}\left(t, p_{\mathrm{i}}, u_{j}, k\right),
\end{gathered}
$$

dove $E V\left(t, p_{\mathrm{i}}\right)$ è il valore atteso al livello della "valuation exposure" calcolato in un intervallo di possibili valori. Si può facilmente vedere come questo secondo metodo sia più generale, dato che si riduce al primo quando $F V\left(t, p_{\mathrm{i}}\right)=E V\left(t, p_{\mathrm{i}}\right)$. Si noti che gli istituti possono applicare indistintamente uno dei due metodi nel calcolare l'AVA. Le linee guida proposte dalla Commissione Rischio di Mercato utilizzano il primo metodo nelle loro applicazioni.

Per quanto riguarda il secondo gruppo di AVA (UCS e IFC), non viene fornita una formula per il calcolo diretto, in quanto questi AVA sono suddivisi nelle loro componenti MPU, CoCo e MoRi e aggregate all'AVA corrispondente.

Si fa notare come tale scomposizione e aggregazione facciano sì che gli AVA UCS e IFC beneficino dello sconto del $50 \%$ relativo agli AVA MPU, CoCo e MoRi. Tuttavia, tale beneficio non è espressamente previsto negli EBA RTS, ma è una diretta conseguenza della loro applicazione. Infatti, l'esclusione degli AVA UCS e IFC da tale beneficio di diversificazione condurrebbe a delle inconsistenze tra il calcolo diretto degli AVA MPU, CoCo e MoRi (pesati al 50\%) e i corrispondenti componenti MPU, CoCo e MoRi derivanti dal calcolo degli AVA UCS e IFC (pesati al 100\%).

Infine, per il terzo gruppo di AVA (CoPo, FAC, EaT e OpR) gli EBA RTS prevedono degli specifici metodi computazionali che analizzeremo in seguito. Si noti che nessun beneficio di diversificazione è previsto per questo terzo gruppo di AVA.

Una volta che sono stati calcolati i 9 AVA previsti dalla normativa, l'AVA totale è calcolato attraverso il seguente algoritmo.

- Gli AVA CoPo, FAC, EaT e OpR sono aggregati ognuno come somma dei componenti individuali e pesati al 100\%.

- Gli AVA UCS e IFC sono scomposti nei loro tre componenti relativi a MPU, CoCo e MoRi, in modo da ottenere 2 MPU + 2 CoCo +2 Mori $=6$ componenti AVA individuali da includere negli AVA MPU, CoCo e MoRi totali.

- Gli AVA MPU, CoCo e MoRi sono aggregati come somma:

- delle componenti individuali a livello di singola posizione,

- delle corrispondenti componenti relative agli AVA UCS e IFC,

- di tutte le componenti di cui ai punti precedenti preventivamente pesati al 50\% ai fini di diversification benefit.

- L'AVA totale è calcolato come somma semplice degli AVA MPU, CoCo, MoRi, CoPo, FAC, EaT, OpR residui. 
In conclusione, l'aggregazione finale include il 50\% degli AVA MPU, MoRi, CoCo, UCS e IFC (5 AVA su 9) e il 100\% degli AVA CoPo, FAC, EaT e OpR (4 AVA su 9).

La Tabella 2 mostra un esempio di calcolo dell'AVA nel "core approach". Gli AVA IFC e UCS sono suddivisi nelle loro rispettive componenti MPU, CoCo e MoRi e preventivamente aggregati agli AVA corrispondenti. L'AVA totale è, infine ottenuto dall'aggregazione degli altri sette AVA. Al fine di presentare un esempio realistico, l'AVA OpR è stato calcolato per un istituto non-AMA. L'ultima riga, definisce un possibile valore dell'AVA fall-back per il rimanente $1 \%$ x $0.1 \%$ del fair value totale.

\begin{tabular}{|c|c|c|c|c|c|c|}
\hline \multicolumn{7}{|c|}{ Core approach } \\
\hline Instrument & Fair Value & $\begin{array}{l}\text { Accounting } \\
\text { reserve }\end{array}$ & $\begin{array}{l}\text { Prudential } \\
\text { filter }\end{array}$ & $\begin{array}{c}\text { Fair Value } \\
\text { for AVA }\end{array}$ & Category level AVAs & Value \\
\hline Derivatives HFT BTB & $14,000.00$ & 0.00 & $0 \%$ & 0.00 & AVA Market price uncertainty (MPU) & 2.91 \\
\hline Derivatives HFT & $12,000.00$ & 0.00 & $100 \%$ & $12,000.00$ & Investing and funding costs MPU & 0.97 \\
\hline Bonds AFS & $12,000.00$ & -120.00 & $20 \%$ & $2,400.00$ & Unearned credit spread MPU & 0.97 \\
\hline FV Option & $5,000.00$ & 0.00 & $100 \%$ & $5,000.00$ & Other MPUs & 0.97 \\
\hline Bonds AFS EU govies & $2,000.00$ & -4.00 & $0 \%$ & 0.00 & AVA Close out costs ( $\mathrm{CoCo})$ & 2.91 \\
\hline O.I.C.R & $1,000.00$ & 0.00 & $100 \%$ & $1,000.00$ & Investing and funding costs CoCo & 0.97 \\
\hline \multirow[t]{13}{*}{ Total portfolio } & $46,000.00$ & -124.00 & & $20,400.00$ & Unearned credit spread CoCo & 0.97 \\
\hline & & & & & Other CoCos & 0.97 \\
\hline & & & & & AVA Model risk (MoRi) & 2.91 \\
\hline & & & & & Investing and funding costs MoRi & 0.97 \\
\hline & & & & & Unearned credit spread MoRi & 0.97 \\
\hline & & & & & Other MRs & 0.97 \\
\hline & & & & & AVA Concentrated positions (CoPo) & 2.91 \\
\hline & & & & & AVA Future administrative costs (FAC) & 2.91 \\
\hline & & & & & AVA Early termination (EaT) & 2.91 \\
\hline & & & & & AVA Operational risk (OpR) & 0.58 \\
\hline & & & & & AVA core & 18.05 \\
\hline & & & & & AVA fall back & 12.61 \\
\hline & & & & & AVA total & 30.66 \\
\hline
\end{tabular}

Data in $€ / \mathrm{mln}$

Tabella 2: Esempio di calcolo dell'AVA nel "core approach"

\section{Fall-back approach}

La presente sezione descrive un esempio pratico di calcolo del fall-back approach, così come definito nella sezione 4 di questo articolo. In particolare, il calcolo dell'AVA fall-back è eseguito tenendo conto della seguente formula,

$$
\begin{gathered}
A V A=100 \% N U P^{+}+10 \% N_{D e y}+25 \%\left|F V-N U P^{+}\right|_{\text {Non }-D e y x} \\
N U P^{+}:=\max \left[\sum_{i=1}^{N_{f b}} N U P_{i}, 0\right] x \\
N_{D e y}=\sum_{i=1}^{N_{f b}}\left|N_{i}\right|, \quad F V=\sum_{i=1}^{N_{f b}} F V_{i},
\end{gathered}
$$

dove $N_{i}$ rappresenta il valore teorico delle posizioni, $N U P_{\mathrm{i}}, i=1_{s, \ldots}, N_{f b}$ sono i "net unrealized profit" individuali delle posizioni, o le variazioni nei fair value dall'inizio delle negoziazioni, determinati secondo una base first-in-first-out. Ogni elemento nell'equazione sopra si riferisce solamente agli strumenti finanziari inclusi nell'approccio fall-back.

Nella Tabella 3 viene presentato un esempio di calcolo dell'AVA ipotizzando una applicazione del fall-back ad una piccola porzione $(0.1 \%)$ del portafoglio presentato in Tabella 2. 


\begin{tabular}{|c|c|c|c|c|}
\hline \multicolumn{5}{|c|}{ Fall-back approach } \\
\hline Instrument & Fair Value & $\begin{array}{c}\text { Accounting } \\
\text { reserve }\end{array}$ & $\begin{array}{c}\text { Prudential } \\
\text { filter }\end{array}$ & $\begin{array}{c}\text { Fair Value } \\
\text { for AVA }\end{array}$ \\
\hline Derivatives HFT ВТВ & 0.00 & 0.00 & $0 \%$ & 0.00 \\
\hline Derivatives HFT & 12.00 & 0.00 & $100 \%$ & 12.00 \\
\hline Bonds AFS & 12.00 & -1.20 & $20 \%$ & 2.40 \\
\hline FV Option & 0.00 & 0.00 & $100 \%$ & 0.00 \\
\hline Bonds AFS EU govies & 0.00 & -0.04 & $0 \%$ & 0.00 \\
\hline O.I.C.R & 0.00 & 0.00 & $100 \%$ & 0.00 \\
\hline Total portfolio & 24.00 & -1.24 & & 14.40 \\
\hline \multicolumn{2}{|c|}{ Accounting data } & & \multicolumn{2}{|r|}{ Value } \\
\hline \multicolumn{2}{|c|}{ Net Unrealised P\&L derivatives } & & \multicolumn{2}{|r|}{0.01} \\
\hline \multicolumn{2}{|c|}{ Net Unrealised P\&L bonds } & & \multicolumn{2}{|r|}{0.00} \\
\hline \multicolumn{2}{|l|}{ Notional derivatives } & & \multicolumn{2}{|r|}{120.00} \\
\hline \multicolumn{2}{|c|}{ Total FV non derivatives } & & \multicolumn{2}{|r|}{2.40} \\
\hline \multicolumn{2}{|l|}{ AVA data } & & $\%$ & Value \\
\hline \multicolumn{2}{|c|}{ Total Net Unrealised Profit (NUP) } & & $100 \%$ & 0.01 \\
\hline \multicolumn{2}{|l|}{ Notional derivatives } & & $10 \%$ & 12.00 \\
\hline \multirow{2}{*}{\multicolumn{2}{|c|}{ FV-NUP non-derivatives }} & & $25 \%$ & 0.60 \\
\hline & & \multicolumn{2}{|r|}{ AVA fall back } & 12.61 \\
\hline
\end{tabular}

Tabella 3: Esempio di calcolo dell'AVA per le posizioni soggette al fall-back approach. Si ipotizza l'applicazione del fall-back approach ad una porzione pari allo 0,1\% dei portafogli Derivatives HFT e Bonds AFS definiti in Tabella 2. Inoltre, si ipotizza che i net unrealized profit \& losses per derivati e bond siano lo $0,1 \%$ dei corrispondenti valori aggregati, con un valore positivo di P\&l (profitto) per i derivati e negativo (perdita) per i bond. Si fa, poi, l'ipotesi che il valore teorico dei derivati sia 10 volte il fair value.

\section{Esempi di calcolo dell'AVA nel "core approach"}

Nella presente sezione viene introdotto il calcolo degli AVA per ognuna delle 9 categorie. Per ognuna di esse viene fornita un'analisi generale insieme ad un esempio concreto di calcolo. Per un'analisi completa si rimanda alle linee guida AIFIRM.

\subsection{AVA Market Price Uncertainty (MPU)}

L'AVA MPU prende in considerazione il rischio di valutazione derivante dall'incertezza in un input di valutazione. Tale rischio è piuttosto comune nella valutazione dei prezzi e può emergere quando:

- lo strumento finanziario è marked-to-market (es. "contributed securities") e diversi intermediari mostrano prezzi diversi, o il prezzo è illiquido per alcuni contributori;

- lo strumento finanziario è marked-to-model (es. derivato OTC o una non-contributed security) usando alcuni input di valutazione (es. curve dei rendimenti multiple basate sulle quotazioni di mercato degli IRS) e ci sono intermediari multipli per tale input (es. nel mercato ci sono diversi market maker per gli IRS).

Il calcolo dell'AVA MPU può essere effettuato seguendo due diverse metodologie:

- Revaluation approach, che richiede una nuova valutazione del fair value della posizione utilizzando il prudent value del fattore di rischio rilevante. L'additional partial valuation adjustment APVA $A_{M P U}$ è poi calcolato come

$$
A P V A_{M P U}\left(t, p_{\mathrm{i}}, u_{j}\right)=w_{M P U}\left[F V_{M P U}\left(t, p_{\mathrm{i}}, u_{j}\right)-P V\left(t, p_{\mathrm{i}}, u_{j}\right)\right]
$$

- Sensitivity approach, basato su un'espansione in serie di Taylor del fair value $F V\left(t, p_{i}, u_{j}\right)$ attorno al fattore di rischio $u_{j}$,

$$
\begin{gathered}
P V_{M P U}\left(t, p_{\mathrm{i}}, u_{j}\right)=F V_{M P U}\left(t, p_{\mathrm{i}}, \hat{u}_{j}\right)=F V\left(t, p_{\mathrm{i}}, u_{j}\right)-\left|\frac{\partial F V}{\partial u_{j}}\left(t, p_{\mathrm{i}}, u_{j}\right)\left(\hat{u}_{j}-u_{j}\right)\right| x \\
A P V A_{M P U}\left(t, p_{\mathrm{i}}, u_{j}\right)=w_{M P U}\left|\frac{\partial F V}{\partial u_{j}}\left(t, p_{i}, u_{j}\right)\left(\hat{u}_{j}-u_{j}\right)\right|
\end{gathered}
$$


Infine, l'AVA totale per la categoria è calcolato come,

$$
A V A_{M P U}(t)=\sum_{i=1}^{N_{D}} \sum_{j=1}^{\mathbb{N}_{u}} A P V A_{M P U}\left(t, p_{i}, u_{j}\right) .
$$

Si noti che il l'AVA totale della categoria dipende fortemente dalla scelta dei due parametri di calcolo del singolo $A P V A_{M P U}\left(t, p_{i}, u_{j}\right)$ (posizioni $\left\{p_{1}, \ldots, p_{N_{D}}\right\}$ e input $\left\{u_{1}, \ldots, u_{N_{L}}\right\}$ ).

La scelta delle posizioni determina il valore dell'esposizione, il quale può essere definito implicitamente a partire dalla posizione stessa (revaluation approach), o implicitamente a partire dalla sensitivity $\frac{\partial F V}{\partial u_{j}}\left(t_{s} p_{\mathrm{i}}, u_{j}\right)($ sensitivity approach).

\section{Case study \#1: Calcolo dell'AVA MPU per titoli quotati}

In questo esempio si considera un titolo per il quale sono disponibili diversi intermediari e si calcola l'AVA tenendo presente un livello di confidenza del $90 \%$ per quanto riguarda la distribuzione del prezzo di mercato. Nella tabella in alto a sinistra della Figura 4 sono mostrati i 12 prezzi "bid" e "ask" di mercato e i corrispondenti mid-price. Si fa l'ipotesi di calcolo del FV come media dei mid-price (162.25). Nella tabella in alto a destra, viene calcolata la distribuzione dei "mid-price", ordinando i prezzi "bid" di mercato e assegnando il percentile corrispondente. Il "prudent value" per le posizioni lunghe (FV positivo) e corte (FV negativo) è il valore più vicino tra il decimo e il novantesimo percentile (161,97 e -162,49, rispettivamente). Infine, si calcola l'AVA MPU come metà della differenza tra il FV e il PV $(0,14$ e 0,12 per la posizione lunga e corta, rispettivamente).

Si fa notare che il calcolo dell'AVA è possibile grazie all'esistenza di diversi price contributor per lo stesso strumento finanziario. La distribuzione dei prezzi è comunque fortemente irregolare (si veda il grafico in basso in Figura 4). Questo mette in dubbio la robustezza del livello di confidenza del $90 \%$.

\begin{tabular}{cccccccc}
\multicolumn{8}{c}{ Bond price distribution } \\
\hline Point & Source & Bid & Ask & Mid & \multicolumn{1}{c}{ Bid-Ask Mid-Bid Ask-Mid } \\
\hline 1 & Contr. 1 & 161.61 & 162.34 & 161.97 & 0.73 & 0.37 & 0.37 \\
2 & Contr. 2 & 162.16 & 163.08 & 162.62 & 0.92 & 0.46 & 0.46 \\
3 & Contr. 3 & 161.84 & 162.60 & 162.22 & 0.75 & 0.38 & 0.38 \\
4 & Contr. 4 & 161.66 & 163.05 & 162.36 & 1.39 & 0.70 & 0.70 \\
5 & Contr. 5 & 161.74 & 162.98 & 162.36 & 1.24 & 0.62 & 0.62 \\
6 & Contr. 6 & 162.02 & 162.48 & 162.25 & 0.46 & 0.23 & 0.23 \\
7 & Contr. 7 & 160.91 & 162.48 & 161.69 & 1.57 & 0.79 & 0.79 \\
8 & Contr. 8 & 160.86 & 163.85 & 162.35 & 2.98 & 1.49 & 1.49 \\
9 & Contr. 9 & 162.18 & 162.79 & 162.49 & 0.60 & 0.30 & 0.30 \\
10 & Contr. 10 & 161.68 & 163.00 & 162.34 & 1.32 & 0.66 & 0.66 \\
11 & Contr. 11 & 160.73 & 163.59 & 162.16 & 2.85 & 1.43 & 1.43 \\
12 & Contr. 12 & 161.85 & 162.60 & 162.23 & 0.75 & 0.38 & 0.38 \\
\hline Average & & 161.60 & 162.90 & 162.25 & 1.30 & 0.65 & 0.65 \\
\hline
\end{tabular}

\begin{tabular}{|c|c|c|c|c|c|c|}
\hline \multicolumn{7}{|c|}{ Market price uncertainty } \\
\hline Point & Mid & Rank & Percent & Bin & Freq. & Cumul. $\%$ \\
\hline 2 & 162.62 & 1 & $100.00 \%$ & 161.69 & 1 & $8.33 \%$ \\
\hline 9 & 162.49 & 2 & $90.90 \%$ & 161.85 & 0 & $8.33 \%$ \\
\hline 5 & 162.36 & 3 & $81.80 \%$ & 162.00 & 1 & $16.67 \%$ \\
\hline 4 & 162.36 & 4 & $72.70 \%$ & 162.16 & 0 & $16.67 \%$ \\
\hline 8 & 162.35 & 5 & $63.60 \%$ & 162.31 & 4 & $50.00 \%$ \\
\hline 10 & 162.34 & 6 & $54.50 \%$ & 162.47 & 4 & $83.33 \%$ \\
\hline 6 & 162.25 & 7 & $45.40 \%$ & 162.62 & 2 & $100.00 \%$ \\
\hline 12 & 162.23 & 8 & $36.30 \%$ & & & \\
\hline 3 & 162.22 & 9 & $27.20 \%$ & & & \\
\hline 11 & 162.16 & 10 & $18.10 \%$ & & & \\
\hline 1 & 161.97 & 11 & $9.00 \%$ & & & \\
\hline 7 & 161.69 & 12 & $0.00 \%$ & & & \\
\hline \multirow[t]{2}{*}{ Long: } & PV & 161.97 & & Short: & PV & -162.49 \\
\hline & AVA & 0.14 & & & AVA & 0.12 \\
\hline
\end{tabular}

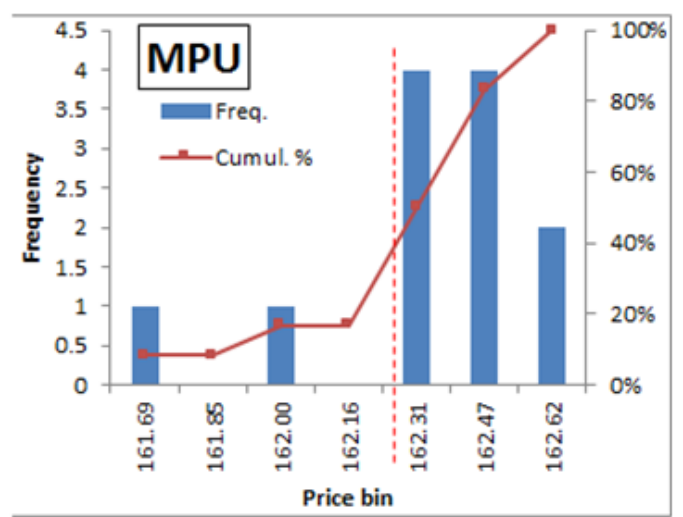

Figura 3: Case study \#1, dati di mercato (in alto a sinistra), distribuzione dell'MPU, prudent value e calcolo dell'AVA MPU (in alto a destra) e grafico della distribuzione (in basso). 
6.2. AVA Close-out costs

L'AVA CoCo si riferisce sia all'incertezza relativa all'"exit price" della posizione, che al costo di liquidità di una certa posizione in una particolare condizione di mercato. Entrambe le situazioni risultano in un aumento del "bid-ask" spread.

Poiché l'illiquidità può essere vista come incertezza circa il mid-price, l'AVA CoCo si sovrappone all'AVA MPU. Dunque, quando l'AVA MPU è basato su prezzi di mercati, l'AVA CoCo deve essere posto uguale a zero.

Anche per l'AVA CoCo sono previsti sia il "revaluation" che il "sensitivity approach". Nel primo caso, si ricalcola il fair value della posizione utilizzando metà del bid-ask spread prudenziale per ogni fattore di rischio; nel secondo caso si utilizza un'espansione in serie di Taylor del fair value attorno al fattore di rischio. Come per l'AVA MPU, valgono le considerazioni circa la dipendenza dell'AVA CoCo dalla scelta delle posizioni e dei fattori di rischio.

Case study \#2: il calcolo dell'AVA CoCo per titoli quotati

Questo case study è basato sullo stesso titolo utilizzato per l'AVA MPU. La tabella in alto a sinistra della Figura 5 mostra le 12 quotazioni dei mid-bid spread di mercato. A destra sono mostrate le corrispondenti quotazioni dell'ask-mid spread. Il FV è dato dalla media dei mid-price $(162,25)$. Inoltre, nelle tabelle in alto sono calcolate le distribuzioni dei bid-mid e ask-mid spread. I prudent bid (per una posizione lunga) e ask (per una posizione corta) sono dati dal valore più vicino al novantesimo percentile (1,43 in entrambi $\mathrm{i}$ casi, poiché $\mathrm{i}$ prezzi bid e ask sono simmetrici). I prudent value sono dati da $162.25-1.43=160.83 \mathrm{e}-162.25-1.43=-163.68$ per le posizioni lunghe e corte, rispettivamente. Infine, l'AVA CoCo è dato da metà della differenza tra FV e PV ( 0,71 sia per long che per short position, sempre per la simmetria). Inoltre, anche in questo caso la distribuzione del prezzo è fortemente irregolare, sollevando gli stessi problemi di robustezza dell'intervallo di confidenza del $90 \%$.

\begin{tabular}{ccccccc}
\multicolumn{7}{c}{ Close Out Costs (long) } \\
\hline Point & Mid-Bid & Rank & Percent & Bin & Freq. Cumul. $\%$ \\
\hline 8 & 1.49 & 1 & $100.00 \%$ & 0.23 & 1 & $8.33 \%$ \\
11 & 1.43 & 2 & $90.90 \%$ & 0.44 & 4 & $41.67 \%$ \\
7 & 0.79 & 3 & $81.80 \%$ & 0.65 & 2 & $58.33 \%$ \\
4 & 0.70 & 4 & $72.70 \%$ & 0.86 & 3 & $83.33 \%$ \\
10 & 0.66 & 5 & $63.60 \%$ & 1.07 & 0 & $83.33 \%$ \\
5 & 0.62 & 6 & $54.50 \%$ & 1.28 & 0 & $83.33 \%$ \\
2 & 0.46 & 7 & $45.40 \%$ & 1.49 & 2 & $100.00 \%$ \\
3 & 0.38 & 8 & $27.20 \%$ & & & \\
12 & 0.38 & 8 & $27.20 \%$ & & & \\
1 & 0.37 & 10 & $18.10 \%$ & & & \\
9 & 0.30 & 11 & $9.00 \%$ & & & \\
6 & 0.23 & 12 & $0.00 \%$ & & \\
\hline Long: & PV & 160.83 & & \\
& AVA & 0.71 & & & \\
\hline \multicolumn{7}{c}{}
\end{tabular}

\begin{tabular}{ccccccc}
\multicolumn{7}{c}{ Close Out Costs (short) } \\
\hline Point & Ask-Mid & Rank & Percent & Bin & Freq. Cumul. \% \\
\hline 8 & 1.49 & 1 & $100.00 \%$ & 0.232 & 1 & $8.33 \%$ \\
11 & 1.43 & 2 & $90.90 \%$ & 0.441 & 4 & $41.67 \%$ \\
7 & 0.79 & 3 & $81.80 \%$ & 0.651 & 2 & $58.33 \%$ \\
4 & 0.70 & 4 & $72.70 \%$ & 0.861 & 3 & $83.33 \%$ \\
10 & 0.66 & 5 & $63.60 \%$ & 1.071 & 0 & $83.33 \%$ \\
5 & 0.62 & 6 & $54.50 \%$ & 1.281 & 0 & $83.33 \%$ \\
2 & 0.46 & 7 & $45.40 \%$ & 1.491 & 2 & $100.00 \%$ \\
3 & 0.38 & 8 & $27.20 \%$ & & & \\
12 & 0.38 & 8 & $27.20 \%$ & & & \\
1 & 0.37 & 10 & $18.10 \%$ & & & \\
9 & 0.30 & 11 & $9.00 \%$ & & & \\
6 & 0.23 & 12 & $0.00 \%$ & & & \\
\hline Long: & PV & -163.68 & & \\
7 & AVA & 0.71 & & & \\
\hline \multicolumn{7}{c}{}
\end{tabular}
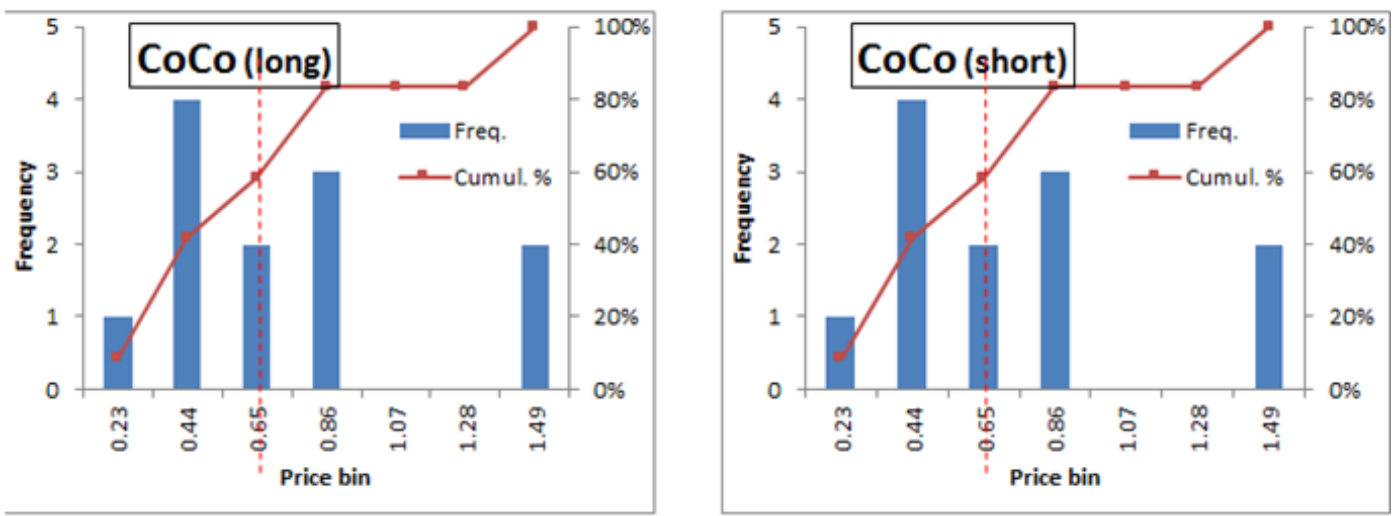

Figura 4: Case study \#1, distribuzione dei dati di mercato CoCo, prudent value e calcolo dell'AVA CoCo (tabelle in alto), grafici delle distribuzioni (in basso). Il FV è dato dalla media dei mid-price $(162,25)$.

\subsection{AVA Model risk (MoRi)}

- L'AVA MoRi si riferisce al rischio derivante dall'incertezza nei modelli utilizzati per la valutazione dei prodotti finanziari non lineari e per la calibrazione di questi su dati di mercato. L'AVA MoRi va calcolato a livello aggregato, includendo tutte le posizioni valutate con il medesimo modello, siano esse plain vanilla o esotiche. Non si considera il singolo deal in modo da sfruttare il beneficio derivante dalle coperture naturali del portafoglio, che possono ridurre il rischio di modello. In generale, poiché i dati e la struttura del problema non permettono di recuperare un livello di informazione tale da raggiungere il livello di confidenza richiesto (90\%), occorre ricorrere ad un approccio expertbased. Tra le possibili alternative, si ricordano: 
- Model risk scenarios: consiste nel generare un insieme di $\mathrm{N}$ scenari alternativi per i dati di mercato disponibili (utilizzando, ad esempio, serie storiche e ricalcolando il valore del portafoglio per ogni scenario). Gli $\mathrm{N}$ valori di portafoglio risultanti sono poi ordinati in una distribuzione di profitti e perdite rispetto al valore dello scenario di base e all'appropriato percentile della distribuzione delle perdite.

- Model stress test: consiste nel far variare direttamente i parametri del modello in modo da estrarne un AVA MoRi con un livello di confidenza del $90 \%$ rispetto alla corrispondente distribuzione degli "stressed price". In realtà, si tratta di un caso particolare dell'approccio a scenari sopra discusso, in quanto fare uno "stress test" sui parametri è circa uguale ad utilizzare diverse alternative di calibrazione. Chiaramente la scelta dei parametri su cui operare richiede un giudizio expert-based e rappresenta un passaggio delicato. I parametri di modello non sono infatti variabili indipendenti, ma sono vincolati dalla procedura di calibrazione al fine di garantire un certo livello di consistenza tra modello e prezzi di mercato.

- Model regression: si tratta di un caso particolare di stress test (e quindi di scenario approach) che consiste nel fissare alcuni parametri del modello in modo da generare un modello più semplice.

- Illiquid model parameters: in certi casi, alcuni parametri di modello possono risultare particolarmente difficili da calibrare in quanto le relative quotazioni sono illiquide o non disponibili. Esempi sono le correlazioni nelle basket option, quanto adjustment, path dependent interest rate payoff, ecc. In questi casi, un possibile approccio alla stima del rischio di modello è quello di utilizzare un'analisi storica.

- Payoff regression: in alcuni casi, si può fare una regressione del payoff dello strumento su payoff meno complessi in modo che il prezzo utilizzato nella regressione funga da vincolo per il prezzo originario. Un caso di questo genere si ha quando uno strumento può essere super/sub replicato utilizzando combinazioni di strumenti plain vanilla. Ad esempio, il prezzo di una Bermudan Swaption è sempre vincolato tra il prezzo della massima European Swaption sottostante e il prezzo del pacchetto di tutte le European Swaption sottostanti.

\subsection{AVA Unearned Credit Spread (UCS)}

L'AVA UCS fa riferimento all'incertezza valutativa che emerge nel credit valuation adjustment (CVA) al fine di includere, date le regole contabili applicabili, il valore calcolato conservativamente delle perdite attese dovute al default della controparte in contratti derivati. Il calcolo di tale aggiustamento corrisponde a un prodotto derivato non lineare di tipo "ibrido". La non linearità dipende dal fatto che l'esposizione rappresenta solo la parte positiva del valore del portafoglio, e la natura "ibrida" è che l'opzione può essere esercitata solo se si manifesta un altro rischio, il default della controparte. Per questo, l'incertezza valutativa di questo prodotto si ripartisce, a sua volta, nelle componenti MPU, CoCo e MoRi. Tali componenti vanno poi aggregate agli AVA corrispondenti.

La formula per il calcolo dell'AVA UCS unilaterale (uCVA) è la seguente,

$$
u C V A_{c}(t) \simeq-L G D_{c}(t) \sum_{\alpha=1}^{N_{T}} q_{c}\left(t, t_{\alpha-1}, t_{\alpha}\right) E P E_{c}\left(t, t_{\alpha}\right),
$$

dove,

- $L G D_{c}(t)=$ Loss Given Default al tempo $t$;

- $E P E_{c}\left(t, t_{\alpha}\right)=$ Expected Positive Exposure al tempo $t_{\alpha}$ atteso al tempo $t<t_{\alpha}$;

- $q_{c}\left(t_{s} t_{\alpha-1}, t_{\alpha}\right)=$ risk neutral marginal default probability.

Nella formula sopra si assume assenza di correlazione tra l'esposizione e il default della controparte: in altri termini, non viene considerato quello che viene chiamato "wrong way risk". Tipicamente, le esposizioni attese sono stimate utilizzando un approccio Monte Carlo, nel quale l'evoluzione dei fattori di rischio sottostante è modellata attraverso dei processi stocastici calibrati sul mercato, mentre le probabilità di default sono estrapolate dai Credit Default Swap (CDS). Dunque, i possibili AVA collegati alla formula sopra definita sono i seguenti:

a. AVA UCS derivante dall'incertezza nelle esposizioni;

b. AVA UCS derivante dall'incertezza nelle probabilità di default;

c. AVA UCS derivante dal model risk, relativo alle ipotesi del modello e ai metodi numerici utilizzati.

Case study \#3: AVA UCS per Interest Rate Swaps e Swaption

Per illustrare la procedura di calcolo e i valori che si ottengono per questo AVA consideriamo i seguenti strumenti finanziari.

1. IRS, payer, ATM, maturità 30Y

2. IRS, payer, ITM, maturità $30 \mathrm{Y}$

3. IRS, payer, OTM, maturità $30 \mathrm{Y}$

4. IRS, payer, ATM, maturità 30Y, con clausola di break-up a 8 anni

5. IRS, payer, ITM, maturità $30 \mathrm{Y}$, con clausola di break-up a 8 anni

6. IRS, payer, OTM, maturità $30 \mathrm{Y}$, con clausola di break-up a 8 anni

7. Swaption, payer, ATM, $5 \mathrm{X} 25 \mathrm{Y}$ 
8. Swaption, payer, ITM, 5X25Y

9. Swaption, payer, OTM, 5X25Y

Completiamo la descrizione dei prodotti con le seguenti informazioni,

- Controparte: Barclays Bank

- Valore nominale: $€ 100 \mathrm{mil}$

- Data di valutazione: $31 / 03 / 2014$

- Garanzie alternative:

- CSA perfetto con soglia zero e "minimum transfer amount" per tutti gli strumenti di cui sopra.

- Nessun CSA per gli strumenti 1, 2, 3, 7, 8, e 9 di cui sopra.

- CSA parziale con soglia di $€ 10$ milioni e "minimum transfer amount" di $€ 1$ milione per gli strumenti 1,2 e 3 di cui sopra.

- Lo scenario base per il calcolo del base value per l' uCVA è il seguente.

- Tassi di interesse: modello Hull-White a un fattore (HW1).

○ Probabilità di default: "bootstrapping" dagli strumenti di calibrazione, ipotizzando un LGD deterministico e costante.

- Strumenti di calibrazione: Swaptions at-the-money (ATM), CDS.

○ Approccio numerico: simulazione Monte Carlo multi-step.

Tutti i calcoli sono stati realizzati utilizzando Numerix CrossAsset XL [11].

I calcoli possono essere riassunti come segue.

L'AVA UCS dovuto all'incertezza nelle esposizioni può essere stimato utilizzando le "stressed exposure", calibrate su scenari di stress della volatilità del mercato swaption. Tale scenario di stress è ottenuto aggiungendo alle volatilità ATM di mercato il $90^{\circ}$ percentile delle variazioni nelle volatilità storiche (orizzonte di due anni, osservazioni bisettimanali). L’AVA corrispondente è dato da

$$
A V A_{1}=\max \left(0, u C V A_{\text {gtressexp }}-u C V A\right)
$$

dove $u C V A_{\text {stressexp }}$ indica il CVA unilaterale con le "stressed exposure".

1. L'AVA UCS dovuto all'incertezza nelle PD può essere stimato utilizzando le "stressed PD" calibrate aggiungendo agli spread CDS il $90^{\circ}$ percentile delle variazioni nei CDS spread (orizzonte di due anni, osservazioni bi-settimanali). L'AVA corrispondente è dato da

$$
A V A_{2}=\max \left(0, u C V A_{s t r e s g}-u C V A\right)_{x}
$$

dove, $u C V A_{\text {stresspD }}$ denota il CVA unilaterale con le stressed PD.

2. L'AVA UCS dovuto al rischio di modello ha due componenti:

- Rischio di modello dovuto all'incertezza del modello utilizzato per stimare le esposizioni,

- Rischio di modello dovuto alle ipotesi nella formula dell'uCVA.

Il primo componente dell'AVA è stimato utilizzando un modello Hull-White (HW2) a due fattori.

$$
A V A_{a}=\max \left(0, u C V A_{M \circ R i}-u C V A\right)_{x}
$$

dove $u C V A_{M \circ R i}$ denota il CVA unilaterale dovuto al rischio di modello.

3. L'AVA UCS è ovviamente influenzato dall'ipotesi che l'esposizione alla controparte sia correlata con il default di quest'ultima, cioè il "wrong way risk" (WWR). Ciò implica la seguente formula,

$$
A V A_{4}=\max \left(0, u C V A_{W W R}-u C V A\right) \text {. }
$$

dove, $u C V A_{W W R}$ è l'uCVA tenendo conto del wrong-way risk.

L'AVA UCS totale è la somma dei quattro AVA calcolati negli step precedenti. Si noti che il peso di 0,5 , presente nella definizione di AVA, non è stato qui considerato. I risultati di tali calcoli sono mostrati nella Tabella 4. 


\begin{tabular}{|c|c|c|c|c|c|c|}
\hline \multicolumn{7}{|c|}{ NO CSA } \\
\hline Instrument & UCVA & $\begin{array}{l}\mathrm{AVA}_{1}-\mathrm{AVA} \\
\text { uncertanty of } \\
\text { exposures }\end{array}$ & $\begin{array}{l}\mathrm{AVA}_{2}-\mathrm{AVA} \\
\text { uncertanty of } \\
\text { PDs }\end{array}$ & $\begin{array}{c}\text { AVA }_{3} \text { - AVA Model Risk: } \\
\text { exposures estimated } \\
\text { using HW2F }\end{array}$ & $\begin{array}{c}\text { AVA }_{4} \text { - AVA Model } \\
\text { Risk: inclusion of } \\
\text { WWR }\end{array}$ & $\begin{array}{c}\text { Total } \\
\text { AVA CVA }\end{array}$ \\
\hline $\begin{array}{c}\text { ATM IRS Pay Fix } \\
30 Y\end{array}$ & 3.259 .456 & 141.254 & 339.644 & $0=\max (0,-701.801)$ & 171.394 & 652.292 \\
\hline $\begin{array}{c}\text { ITM IRS Pay Fix } \\
30 Y\end{array}$ & 4.120 .927 & 133.692 & 439.399 & $0=\max (0,-673.776)$ & 179.032 & 752.123 \\
\hline $\begin{array}{c}\text { OTM IRS Pay Fix } \\
30 Y \\
\end{array}$ & 2.541 .280 & 142.063 & 258.670 & $0=\max (0,-693.465)$ & 154.475 & 555.208 \\
\hline $\begin{array}{c}\text { ATM IRS Pay Fix } \\
30 Y \text { with break } \\
\text { clause at } 8 Y\end{array}$ & 1.242 .162 & 50.361 & 161.081 & $0=\max (0,-189.912)$ & 189.238 & 400.680 \\
\hline $\begin{array}{l}\text { ITM IRS Pay Fix } \\
30 Y \text { with break } \\
\text { clause at } 8 Y\end{array}$ & 1.707 .628 & 45.322 & 224.913 & $0=\max (0,-171.260)$ & 281.077 & 551.312 \\
\hline $\begin{array}{c}\text { OTM IRS Pay Fix } \\
30 Y \text { with break } \\
\text { clause at } 8 Y\end{array}$ & 884.056 & 50.588 & 113.007 & $0=\max (0,-199.830)$ & 102.800 & 266.395 \\
\hline $\begin{array}{c}\text { ATM Swaption } 5 Y \\
\text { to enter into IRS } \\
\text { Pay Fix } 25 Y\end{array}$ & 2.414 .338 & 125.055 & 266.253 & $0=\max (0,-658.330)$ & 149.899 & 541.208 \\
\hline $\begin{array}{c}\text { ITM Swaption } 5 Y \\
\text { to enter into IRS } \\
\text { Pay Fix } 25 Y\end{array}$ & 3.256 .435 & 124.521 & 358.440 & $0=\max (0,-733.575)$ & 111.896 & 594.857 \\
\hline $\begin{array}{l}\text { OTM Swaption } 5 \mathrm{Y} \\
\text { to enter into IRS } \\
\text { Pay Fix } 25 \mathrm{Y}\end{array}$ & 1.718 .934 & 125.736 & 190.173 & $0=\max (0,-606.870)$ & 183.673 & 499.581 \\
\hline
\end{tabular}

\begin{tabular}{|c|c|c|c|c|c|c|}
\hline \multicolumn{7}{|c|}{ CSA with 0 Threshold and 0 Minimum Transfer Amount } \\
\hline Instrument & UCVA & $\begin{array}{c}\text { AVA }_{1} \text { - AVA } \\
\text { uncertanty of } \\
\text { exposures }\end{array}$ & $\begin{array}{c}\text { AVA }_{2} \text { - AVA } \\
\text { uncertanty of } \\
\text { PDs }\end{array}$ & $\begin{array}{c}\text { AVA }_{3} \text { - AVA Model Risk: } \\
\text { exposures estimated } \\
\text { using HW2F }\end{array}$ & $\begin{array}{c}\text { AVA }_{4} \text { - AVA Model } \\
\text { Risk: inclusion of } \\
\text { WWR }\end{array}$ & $\begin{array}{c}\text { Total } \\
\text { AVA CVA }\end{array}$ \\
\hline $\begin{array}{c}\text { ATM IRS Pay Fix } \\
\text { 30Y }\end{array}$ & 194.823 & 10.176 & 21.850 & $0=\max (0,-30.678)$ & $0=\max (0,-5.593)$ & 32.026 \\
\hline $\begin{array}{c}\text { ITM IRS Pay Fix } \\
\text { 30Y }\end{array}$ & 186.114 & 9.719 & 20.810 & $0=\max (0,-30.040)$ & $0=\max (0,-5.391)$ & 30.529 \\
\hline $\begin{array}{c}\text { OTM IRS Pay Fix } \\
\text { 30Y }\end{array}$ & 203.535 & 10.634 & 22.891 & $0=\max (0,-31.313)$ & $0=\max (0,-5.791)$ & 33.525 \\
\hline $\begin{array}{c}\text { ATM Swaption 5Y } \\
\text { to enter into IRS } \\
\text { Pay Fix 25Y }\end{array}$ & 79.006 & 3.459 & 8.737 & $0=\max (0,-9.067)$ & $0=\max (0,-159)$ & 12.196 \\
\hline $\begin{array}{c}\text { ITM Swaption 5Y } \\
\text { to enter into IRS } \\
\text { Pay Fix 25Y }\end{array}$ & 97.510 & 3.325 & 10.753 & $0=\max (0,-9.754)$ & $0=\max (0,-2.302)$ & 14.078 \\
\hline $\begin{array}{c}\text { OTM Swaption 5Y } \\
\text { to enter into IRS } \\
\text { Pay Fix 25Y }\end{array}$ & 58.126 & 3.627 & 6.497 & $0=\max (0,-9.861)$ & 6.600 \\
\hline
\end{tabular}




\begin{tabular}{|c|c|c|c|c|c|c|}
\hline \multicolumn{7}{|c|}{ CSA with 10 mio $€$ Threshold and 1 mio $€$ Minimum Transfer Amount } \\
\hline Instrument & UCVA & $\begin{array}{c}\text { AVA }_{1} \text { - AVA } \\
\text { uncertanty of } \\
\text { exposures }\end{array}$ & $\begin{array}{c}\text { AVA }_{2} \text { - AVA } \\
\text { uncertanty of } \\
\text { PDs }\end{array}$ & $\begin{array}{c}\text { AVA }_{3} \text { - AVA Model Risk: } \\
\text { exposures estimated } \\
\text { using HW2F }\end{array}$ & $\begin{array}{c}\text { AVA }_{4} \text { - AVA Model } \\
\text { Risk: inclusion of } \\
\text { WWR }\end{array}$ & $\begin{array}{c}\text { Total } \\
\text { AVA CVA }\end{array}$ \\
\hline $\begin{array}{c}\text { ATM IRS Pay Fix } \\
\text { 30Y }\end{array}$ & 1.226 .799 & 11.556 & 127.905 & $0=\max (0,-96.712)$ & 53.766 & 193.227 \\
\hline $\begin{array}{c}\text { ITM IRS Pay Fix } \\
\text { 30Y }\end{array}$ & 1.433 .338 & 2.477 & 153.022 & $0=\max (0,-5.376)$ & 41.847 & 197.346 \\
\hline $\begin{array}{c}\text { OTM IRS Pay Fix } \\
\text { 30Y }\end{array}$ & 1.021 .404 & 21.445 & 103.709 & $0=\max (0,-103.166)$ & 64.876 & 190.030 \\
\hline
\end{tabular}

Tabella 4: Risultati del calcolo dell'AVA UCS per diversi strumenti

\subsection{AVA Investing and funding costs (IFC)}

L'AVA IFC si riferisce all'incertezza valutativa nei costi di finanziamento (funding cost) utilizzati nella stima dell'exit price della posizione, tenendo conto degli standard contabili applicabili. Anche l'aggiustamento per i funding cost può essere considerato un prodotto non lineare implicito che modifica il valore del prodotto finanziario, e come tale la sua incertezza valutativa può essere scomposta in MPU, CoCo e MoRi.

Per quanto riguarda il calcolo di questo AVA, occorre distinguere tra la valutazione di posizioni in derivati con un CSA quasi perfetto ("strongly collateralized derivatives"), per cui il costo di finanziamento è già incluso nel FV utilizzando la metodologia di sconto OIS (in questo caso $A V A_{I F C}(t)=0$ ) e tutti gli altri casi, per cui occorre considerare se l'istituto include l'FVA nel calcolo del FV. Se ciò non avviene, l' $A V A_{I F C}(t)$ corrisponde all'aggiustamento FVA complessivo calcolato nell'ipotesi di "prudent value".

Per quanto riguarda la suddivisione, si tiene conto delle seguenti incertezze valutative:

- $\quad A V A_{I F C}(t, M P U)$ : incertezze nei fattori di rischio sottostanti il calcolo dell'FVA, come funding spread, probabilità di default e tassi di recupero, e recovery rates e esposizioni sottostanti;

- $\quad A V A_{I F C}(t, C o C o):$ bid/ask nei fattori di rischio di cui sopra;

- $\quad A V A_{I F C}(t, M o R i)$ : incertezze dovute ai modelli e ai metodi numerici utilizzati nel calcolo dell'esposizione, quali la scelta dell'intervallo di tempo discreto per la simulazione, le diverse dinamiche utilizzate per simulare i fattori di rischio sottostanti, etc.

\section{Case study \#4: AVA IFC per Interest Rate e Swaption}

Riportiamo qui l'esempio precedente dei prodotti per i quali abbiamo calcolato l'AVA UCS, a cui si fa riferimento per la descrizione. Il calcolo dell'AVA IFC è stato qui eseguito tenendo conto delle seguenti formule,

$$
\begin{gathered}
F C A_{c}(t) \simeq-\sum_{\alpha=1}^{N_{T}} \Delta F_{I}^{+}\left(t, t_{\alpha-1}, t_{\alpha}\right) \Delta t_{\alpha} s_{I}\left(t, t_{\alpha-1}\right) s_{c}\left(t_{v} t_{\alpha-1}\right) E P E_{c}\left(t_{v} t_{\alpha}\right) x \\
F B A_{c}(t) \simeq-\sum_{\alpha=1}^{N_{T}} \Delta F_{I}^{-}\left(t, t_{\alpha-1}, t_{\alpha}\right) \Delta t_{\alpha} s_{I}\left(t_{v} t_{\alpha-1}\right) s_{c}\left(t, t_{\alpha-1}\right) E N E_{c}\left(t, t_{\alpha}\right), \\
A V A_{I F C}(t)=\sum_{c=1}^{N_{c t p}}\left[F C A_{c}(t)+F B A_{c}(t)\right],
\end{gathered}
$$

dove,

- $\quad F C A_{c}(t)$ (Funding Cost Adjustment, negativo) è il funding cost generato dallo spread tra il tasso di finanziamento e il tasso sul collaterale (quando la banca deposita fondi in garanzia e riceve degli interessi su di essi);

- $\quad F B A_{e}(t)$ (Funding Benefit Adjustment, positive) è il guadagno generato dallo spread tra il tasso di investimento e il tasso sul collaterale (quando la banca riceve fondi in garanzia e paga degli interessi su di esse);

$\circ E_{c} E_{c}\left(t, t_{\alpha}\right), E N E_{c}\left(t, t_{\alpha}\right)=$ Expected Positive/Negative Exposure al tempo $t$ attese al tempo $t<t_{\alpha}$, rispettivamente.

$\circ s_{x}(t, u)=$ probabilità di sopravvivenza a $t<u$ oltre il tempo $u$.

Inoltre, si ipotizza che l'istituto non includa l'FVA nel calcolo del FV, in modo che il "full FVA" sia incluso nella prudent valuation. 


\begin{tabular}{lc}
\hline \multicolumn{1}{c}{ Instrument } & AVA IFC \\
\hline IRS, payer, ATM, maturity 30Y & $1,888,327$ \\
IRS, payer, ITM, maturity 30Y & $5,087,600$ \\
IRS, payer, OTM, maturity 30Y & $-1,308,850$ \\
IRS, payer, ATM, maturity 30Y, break clause at 8Y & $1,271,040$ \\
IRS, payer, ITM, maturity 30Y, break clause at 8Y & $3,483,938$ \\
IRS, payer, OTM, maturity 30Y, break clause at 8Y & $-940,408$ \\
Swaption, payer, ATM, 5X25Y & $4,623,346$ \\
Swaption, payer, ITM, 5X25Y & $6,461,297$ \\
Swaption, payer, OTM, 5X25Y & $3,672,171$ \\
\hline
\end{tabular}

Tabella 5: Calcolo dell'AVA IFC. I calcoli sono stati eseguiti utilizzando una funding curve costante di 300bp.

\subsection{AVA Concentrated Positions (CoPo)}

L'AVA CoPo si riferisce all'incertezza valutativa nell'exit price per posizioni concentrate. In generale, si considerano concentrate tutte quelle posizioni che, dati gli impatti di mercato di un eventuale smobilizzo, hanno il potenziale di produrre perdite tali da minare la stabilità dell'istituto.

Nel calcolo dell'AVA CoPo si distingue tra titoli e derivati. I derivati generalmente non presentano posizioni concentrate. Se tale eventualità si dovesse presentare, la situazione va documentata e si deve calcolare l'AVA CoPo. Per quanto riguarda, invece, i titoli l'AVA CoPo va calcolato come segue:

○ ricercare le posizioni concentrate comparando la quantità di uno stesso titolo detenuta in portafoglio in rapporto al flottante totale;

- stimare i coefficienti di incertezza relativi a tali grandi quantità;

- calcolare l'AVA CoPo valutando la sensitività dei fattori di rischio di credito e le incertezze.

Case study \#5: AVA CoPo per i titoli

In questo case study, viene presentato un esempio di calcolo dell'AVA CoPo per i titoli. Come mostrato in Tabella 6, si ipotizza la presenza in portafoglio di un ammontare importante di un certo titolo (11,5\% del flottante) e si assegna a tale posizione un'incertezza CoPo pari al 10\%, utilizzando un approccio expert-based. Inoltre, utilizzando gli MPU stimati per gli z-spread e la sensitività CR01, si ottiene l'AVA CoPo mostrato nella cella in basso a destra utilizzando la seguente equazione

$$
A V A_{C O P O}(t)=w_{M P U} n|C R 01| \Delta_{M P U} Z .
$$

\begin{tabular}{ccc}
\hline Field & Symbol & Value \\
\hline ISIN & & XXX123 \\
Name & & YYY \\
Issuer & & ZZZ Bank \\
Nominal (€) & $\mathrm{n}$ & $57,500,000$ \\
Outstanding (€) & $\mathrm{N}$ & $500,000,000$ \\
Size held & $\mathrm{n} / \mathrm{N}$ & $11.50 \%$ \\
Uncertainty CoPo (\%) & $\Delta_{M P U}$ & $10 \%$ \\
Z-spread used for pricing (bps) & $\mathrm{Z}$ & 62 \\
Z-spread MPU (bps) & $\Delta_{M P U} \mathrm{Z}$ & 6.2 \\
CR01 ( $€ /$ bps) & $\mathrm{CRO1}$ & -0.011526667 \\
AVA CoPo weight & $W_{M P U}$ & 1 \\
AVA CoPo $(€)$ & & 41092.57 \\
\hline
\end{tabular}

Tabella 6: Esempio di calcolo di AVA CoPo

\subsection{AVA Future Administrative Costs (FAC)}

L'AVA FAC prende in considerazione l'incertezza valutativa che emerge dai possibili costi amministrativi e di copertura relativi a posizioni per le quali non è applicato un "exit price" di mercato per i costi di chiusura. Tali costi sono dunque complementari ai close-out cost. Se questi ultimi sono misurati sulla base di un exit price di mercato (full exit price), allora, dopo aver eseguito la corrispondente strategia di liquidazione, le posizioni scompaiono e non restano "future administrative cost" da calcolare. Se invece i costi di chiusura sono misurati su una base "cost-to-hedge", come per i portafogli di derivati, allora le posizioni di copertura devono essere portate a scadenza generando costi amministrativi futuri.

Il calcolo dell'AVA FAC può venire effettuato tenendo presente il seguente schema: 
- $\quad$ Identificazione delle posizioni pi per cui l'AVA MPU e CoCo non sono calcolati o non sono calcolati secondo una base di full exit price.

- $\quad$ Per le posizioni calcolate al full exit price si ponga $A P V A_{F A C}\left(t_{s} p_{i}\right)=0$.

- Per le posizioni $p_{\mathrm{i}}$ non calcolate al full exit price, si calcoli l' $A P V A_{F A C}\left(t_{s} p_{i}\right)$ tenendo conto dei seguenti step.

$\circ \mathrm{Si}$ calcoli il valore atteso futuro scontato $V_{F A C}\left(t, T_{k} p_{\mathrm{i}}\right)$ della posizione $p_{\mathrm{i}}$ alle date discrete future $t<T_{1, \ldots x} T_{M x}$

- Si calcolino i costi amministrativi e di copertura attesi in $t$ per i futuri intervalli di tempo [ $\left.T_{k-1}, T_{k}\right]$ per unità di valuta, $C\left(t, T_{k}, p_{i}\right)$, .

- Si calcoli l' $A P V A_{F A C}\left(t, p_{i}\right)$ individuale per ogni posizione $p_{\mathrm{i}}$ utilizzando la seguente formula

$$
A P V A_{F A C}\left(t, p_{i}\right)=\sum_{k=T_{1}}^{T_{M}} V_{F A C}\left(t, T_{k}, p_{\mathrm{i}}\right) C\left(t, T_{k}, p_{\mathrm{i}}\right)\left(T_{k}-T_{k-1}\right)
$$

- Si calcoli l'AVA $A_{F A C}(t)$ totale per la categoria come la somma degli $A P V A_{F A C}\left(t, p_{i}\right)$ individuali per ogni categoria,

$$
A V A_{F A C}(t)=\sum_{i} A P V A_{F A C}\left(t, p_{i}\right)
$$

Una formula più semplice considera direttamente l'AVA totale calcolato come media pesata delle maturity,

$$
A V A_{F A C}\left(t, p_{i}\right) \cong V_{F A C}\left(t, T_{\text {avg }}, p_{i}\right)\left(T_{\text {avg }}-t\right) .
$$

Chiaramente i costi amministrativi $V_{F A C}\left(t_{s} T_{k^{s}} p_{\mathrm{i}}\right)$ rappresentano il dato più difficile da ottenere. Generalmente, si è in grado di ottenere i costi amministrativi presenti e si può ipotizzare che essi siano costanti fino a scadenza.

\subsection{AVA Early Termination (EaT)}

L'AVA EaT prende in considerazione l'incertezza valutativa che emerge da perdite potenziali derivanti da chiusure anticipate dai contratti, non previste dai contratti stessi e concordate con i clienti.

Il calcolo dell'AVA EaT deve basarsi sui dati storici e dovrebbe seguire lo schema qui riportato.

- Identificazione della posizione $p_{i}$ soggetta a possibili EaT non contrattuali.

- Identificazione di un finestra temporale ammissibile $\left[T_{s}, t\right]$, dove $T<t$.

- Identificazione di negoziazioni passate $u_{j} j=1_{s \ldots}, N_{E a T}$, soggette a EaT non contrattuali in periodi passati

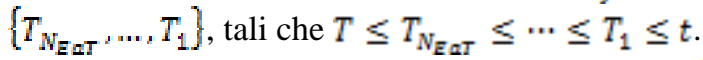

- $\quad$ Recupero dei fair value storici corrispondenti $F V\left(T_{j}, u_{j}\right)$ e i prezzi di liquidazione attuali $P\left(T_{j}, u_{j}\right)$.

- Calcolo delle percentuali di profitti e perdite storiche,

$$
P L\left(T_{j}, u_{j}\right):=\left[P\left(T_{j}, u_{j}\right)-F V\left(T_{j}, u_{j}\right)\right] / F V\left(T_{j}, u_{j}\right)
$$

- Calcolo della distribuzione di profitti e perdite storiche e il $10^{\circ}$ percentile della distribuzione di profitti e perdite, $P L_{1076}$ Il $10^{\circ}$ percentile può essere negativo (Perdita) o positivo (profitto). Il significato è che se si trova che $P L_{1036}=-1 \%$ si può concludere che nel $90 \%$ dei EaT passati la perdita è risultata minore dell $1 \%$ del $\mathrm{FV}$.

- Calcolo dell'APVA EaT individuale attraverso la seguente formula

$$
A P V A_{E a T}\left(t, p_{i}\right)=\left\{\begin{array}{cc}
0, & \text { if } P L_{1036} \geq 0, \\
\left|P L_{1036} \times F V\left(t, p_{i}\right)\right| & \text { if } P L_{1096}<0 .
\end{array}\right.
$$

- Calcolo dell'AVA EaT totale di categoria, come

$$
A V A_{E a T}(t)=\sum_{i} A P V A_{E a T}\left(t, p_{i}\right) .
$$




\subsection{AVA Operational Risk (OpR)}

L'AVA OpR prende in considerazione l'incertezza valutativa che emerge dalle perdite potenziali in cui l'istituto può incorrere a causa di rischi operativi relativi al processo di valutazione. Questo rischio è legato principalmente, ma non solo, alla gestione della riconciliazione contabile e alle possibili dispute legali.

Il principale fattore dell'AVA OpR è legato all'implementazione del rischio operativo adottato dall'istituto. Agli istituti che usano l'Advanced Measurement Approach (AMA) Operational Risk, definito nella CRR, titolo III, Ch. 4, artt.321-324 (istituti AMA), è ammesso il calcolo di un AVA OpR più leggero. Tale facilitazione è atta ad evitare conteggi doppi di riserve di capitale destinate alla stessa fonte di rischio. In particolare, viene posto $A V A_{O p R}(t)=0$ se l'istituto fornisce prova che il rischio operativo relativo al processo di valutazione è pienamente calcolato dall'AMA. In caso contrario, l'istituto deve calcolare l' $A V A_{O p R}$ stimando l'incertezza valutativa derivante dalle perdite potenziali relative al rischio operativo nei processi di valutazione non coperti dall'AMA.

In tutti gli altri casi (istituti non-AMA), l’AVA OpR è dato dal 10\% della somma di AVA MPU e AVA CoCo:

$$
A V A_{O p R}(t)=10 \% \times\left[A V A_{M P U}(t)+A V A_{C o C O}(t)\right]
$$

\section{Il framework operativo della prudent valuation}

Descriviamo un possibile schema operativo per la prudent valuation, tenendo presenti i requisiti regolamentari in termini di ruoli, responsabilità, sistemi e controlli. Considerando la prudent valuation come un processo, si distinguono quattro aree principali: governance, metodologia, documentazione e reporting, tecnologia.

\subsection{Governance}

La governance della prudent valuation richiede che gli istituti finanziari integrino i loro modelli operativi in termini di ruoli, responsabilità e processi di misurazione, gestione e controllo sia delle valutazioni al fair value che di quelle prudenziali. Si possono, dunque, elencare le funzioni che gli istituti devono aggiornarne per adattare la governance di valutazione alla prudent valuation:

- definizione di processi e controlli per la prudent valuation ed implementazione lungo tutta la filiera produttiva;

- definizione del risk appetite per quanto riguarda le esposizioni di posizioni soggette a incertezza valutativa;

- identificazione delle unità coinvolte nel processo di prudent valuation e dei loro rispettivi processi produttivi;

- introduzione dei processi di prudent valuation nel dominio dell'audit interno;

- definizione di documentazione e reporting.

\subsection{Metodologia}

L'art. 18 degli EBA RTS richiede una documentazione appropriata per quanto riguarda le metodologie di calcolo degli AVA. Si rimanda alla lettura della norma per la trattazione completa di questo argomento.

\subsection{Documentazione e reporting}

Per quanto riguarda documentazione e reporting, gli istituti devono costituire, realizzare e mantenere una Prudent Valuation Policy, che includa due sezioni:

- Governance: la documentazione riguardante la governance

- Metodologia: la documentazione riguardante la metodologia

Inoltre, gli istituti devono integrare i loro reporting di valutazione esistenti come segue:

- Regulatory reporting: inclusione degli AVA nella deduzione di CET1 secondo l'art. 34 della CRR.

- Management reporting: le informazioni relative al processo di calcolo degli AVA vanno riportate al senior management, in modo da assicurare un'adeguata comprensione del livello di incertezza valutativa nel portafoglio delle posizioni valutate al fair value dall'istituto (EBA RTS art. 18.2).

\subsection{Tecnologia}

La "prudent valuation" richiede un sistema IT automatizzato per i processi di calcolo e alimentazione. In particolare, il sistema IT deve essere integrato sia ai sistemi contabili che alle piattaforme regolamentari. Inoltre, il sistema IT deve implementare dei dispositivi di calcolo e alimentazione e deve essere in grado di monitorare i dati di input e output. Infine, gli strumenti di management reporting devono essere sviluppati all'interno del sistema di IT.

La costruzione di un sistema di software o di un modulo è basato sulla definizione dei requisiti, che è generalmente abbastanza progressiva e accurata da coprire le scelte importanti nelle prime fasi del progetto e seguire il disegno finale, man mano che i dettagli crescono.

\section{Conclusioni}

In questo articolo abbiamo rfornito una rappresentazione sintetica della regolamentazione sulla Prudent Valuation e delle alternative a disposizione delle banche per l'implementazione. Mentre informazioni di maggiore dettaglio ed esempi di 
applicazione possono essere ricavati dalla consultazione della relazione conclusiva della Commissione Rischio di Mercato dell'AIFIRM, qui abbiamo riportato le domande fondamentali che gli istituti bancari dovranno porsi per rispondere a questo nuovo quesito regolamentare. La scelta sarà agevole per gli istituti bancari di minore dimensione, e di più ridotta esposizione a poste di bilancio valutate al fair value. Per queste sarà sufficiente accantonare a capitale di vigilanza l'uno per mille del valore complessivo del portafoglio a fair value. Per le banche più grandi, e quelle che lo diventeranno a seguito di processi di aggregazione, il calcolo del capitale da accantonare per tener conto del rischio valutativo, il calcolo degli AVA, gli aggiustamenti di valutazione, rappresenteranno un'importante occasione per intraprendere un'analisi analitica degli elementi che determinano questa forma di rischio. In particolare, l'incertezza sui prezzi osservati, e il loro contenuto informativo, e sui prezzi di smobilizzo della posizione, rappresentano le fonti che maggiormente sono collegate all'opacità dei mercati. Il rischio di modello ha poi un impatto rilevante su tutti i prodotti di tipo non lineare, e i prodotti non lineari (noti come XVA, o aggiornamenti di valutazione) che rappresentano il rischio di controparte e di liquidità in contratti derivati e strumenti finanziari. Questi e altri elementi potranno portare gli intermediari a conoscere più da vicino una forma di rischio che ha avuto un ruolo di primo piano in una delle più profonde crisi finanziarie della storia, quella iniziata nel 2008 e che stiamo ancora vivendo.

Marco Bianchetti, Umberto Cherubini e Roberto Tubaldi

Questo articolo è tratto dal documento "Prudent Valuation Guidelines and Sound Practices", Marzo 2016, elaborato a cura della Commissione AIFIRM Rischi di Mercato.

\section{Bibliografia}

[1] Regulation (EU) No 575/2013 of the European Parliament and of the Council of 26 June 2013 on prudential requirements for credit institutions and investment firms and amending Regulation (EU) No 648/2012.

[2] European Banking Authority, "EBA final draft Regulatory Technical Standards Regulatory Technical Standards on prudent valuation under Article 105(14) of Regulation (EU) 575/2013 (Capital Requirements Regulation - CRR)", 23 January 2015, https://www.eba.europa.eu/regulation-and-policy/market-risk/draft-regulatory-technical-standards-onprudent-valuation. Official Journal of the European Union, 28 Jan. 2016, http://eur-lex.europa.eu/legalcontent/EN/TXT/PDF/?uri=OJ:L:2016:021:FULL.

[3] Basel Committee Banking Supervision, "International Convergence of Capital Measurement and Capital Standards - A revised framework", June 2004, http://www.bis.org/publ/bcbs118.htm.

[4] Financial Services Authority, "Dear CEO Letter: Valuation and Product Control”, August 2008, http://www.fsa.gov.uk/pubs/ceo/valuation.pdf.

[5] Financial Services Authority, "Product Control Findings and Prudent Valuation Presentation", November 2010, http://www.fsa.gov.uk/pubs/other/pcfindings.pdf.

[6] Financial Services Authority, "Regulatory Prudent Valuation Return", Policy Statement 12/7, April 2012, http://www.fsa.gov.uk/library/policy/policy/2012/12-07.shtml.

[7] European Banking Authority, "Discussion Paper relating to Draft Regulatory Technical Standards on prudent valuation under Article 100 of the draft Capital Requirement Regulation (CRR)" EBA/DP/2012/03, 13 November 2012, http://www.eba.europa.eu/-/eba-discussion-paper-on-draft-regulatory-standards-on-prudent-valuation.

[8] European Banking Authority, "Consultation Paper Draft Regulatory Technical Standards on prudent valuation under Article 105(34) of Regulation (EU) 575/2013 (Capital Requirements Regulation - CRR)”, EBA/CP/2013/28, 10 July 2013, $\quad$ http://www.eba.europa.eu/regulation-and-policy/market-risk/draft-regulatory-technical-standards-on-prudentvaluation.

[9] European Banking Authority, "Questions and Answers on prudent valuation”, October 2013, http://www.eba.europa.eu//revised-faqs-on-prudent-valuation-q-1.

[10] European Banking Authority, "Quantitative Impact Study on prudent valuation", November 2013, http://www.eba.europa.eu/-/eba-launches-qis-exercise-on-prudent-valuation.

[11] Numerix, http://www.numerix.com. 


\section{Newsletter AIFIRM - Risk Management Magazine}

Anno 11, $\mathrm{n}^{\circ} 1 \quad$ Gennaio - Febbraio - Marzo 2016

Direttore Responsabile:

Maurizio Vallino

\section{Condirettore}

Corrado Meglio

Consiglio Scientifico:

Simona Cosma

Emanuele Diquattro

Paola Ferretti

Giampaolo Gabbi

Fausto Galmarini

Andrea Giacomelli

Pier Giuseppe Giribone

Rossano Giuppa

Aldo Letizia

Corrado Meglio

Paolo Palliola

Enzo Rocca

Fabio Salis

Cristiana Schena

Giuseppe Torluccio

Maurizio Vallino

Vignettista: Silvano Gaggero

Proprietà, Redazione e Segreteria:

Associazione Italiana Financial Industry Risk Managers (AIFIRM), Via Sile 18, 20139 Milano

Registrazione del Tribunale di Milano $n^{\circ} 629$ del 10/9/2004

E-mail: segreteria@ aifirm.it;

Tel. 3896946315

Lunedì - Venerdì h.15-17

Stampa: Algraphy S.n.c. - Passo Ponte Carrega 62-62r

16141 Genova

Le opinioni espresse negli articoli impegnano unicamente la responsabilità dei rispettivi autori

SPEDIZIONE IN ABBONAMENTO POSTALE AI SOCI AIFIRM RESIDENTI IN ITALIA, IN REGOLA CON L'ISCRIZIONE 


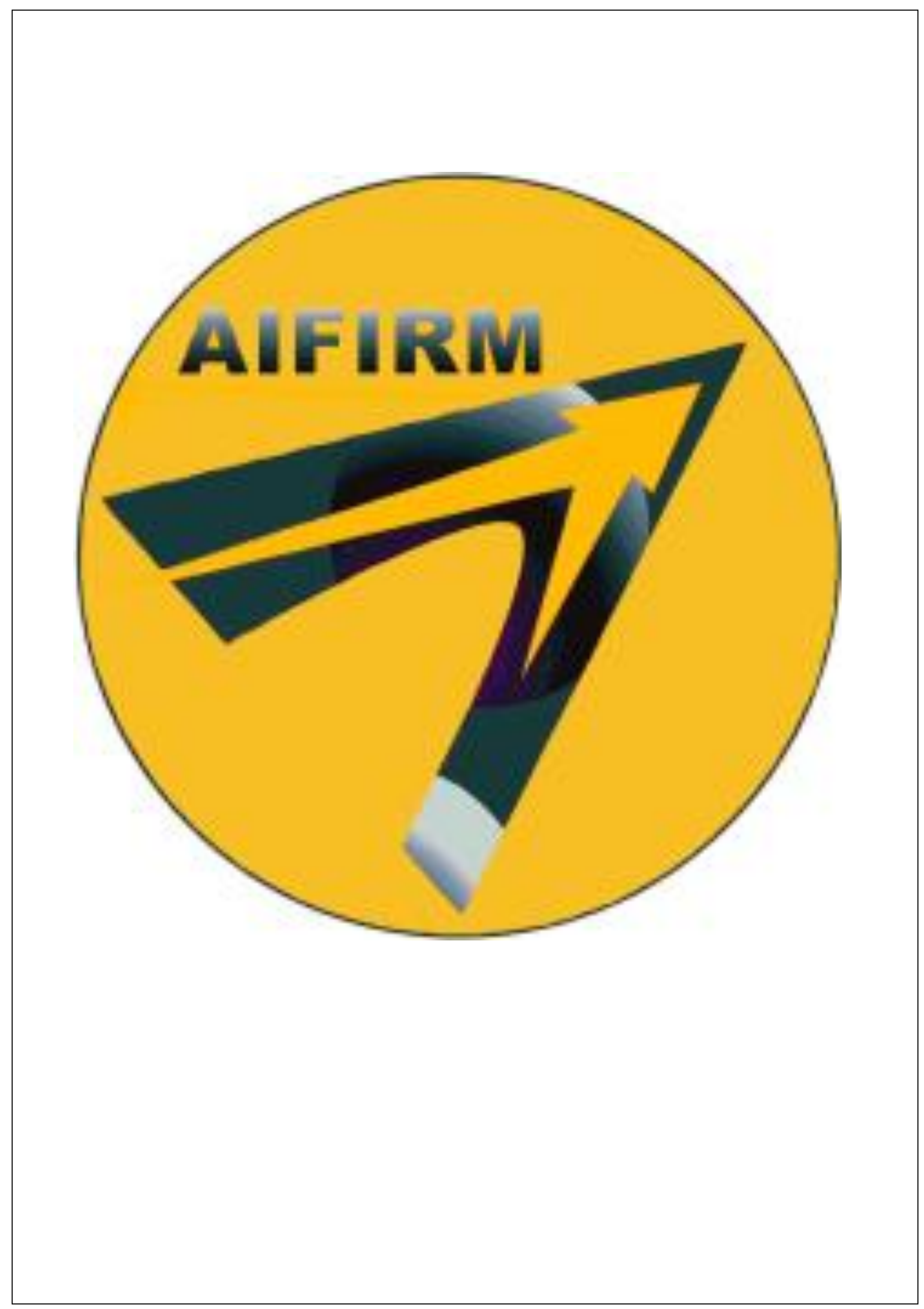

